\title{
DEPENDENT FIRST ORDER THEORIES, CONTINUED
}

\author{
SAHARON Shelah \\ The Hebrew University of Jerusalem \\ Einstein Institute of Mathematics \\ Edmond J. Safra Campus, Givat Ram \\ Jerusalem 91904, Israel \\ Department of Mathematics \\ Hill Center-Busch Campus \\ Rutgers, The State University of New Jersey \\ 110 Frelinghuysen Road \\ Piscataway, NJ 08854-8019 USA
}

\begin{abstract}
A dependent theory is a (first order complete theory) $T$ which does not have the independence property. A major result here is: if we expand a model of $T$ by the traces on it of sets definable in a bigger model then we preserve its being dependent. Another one justifies the cofinality restriction in the theorem (from a previous work) saying that pairwise perpendicular indiscernible sequences, can have arbitrary dual-cofinalities in some models containing them. We introduce "strongly dependent $T$ " and look at definable groups in such models; also look at forking and relatives.
\end{abstract}

The author would like to thank the Israel Science Foundation for partial support of this research (Grant No. 242/03). Publication 783.

I would like to thank Alice Leonhardt for the beautiful typing.

Typeset by $\mathcal{A} \mathcal{M S}-\mathrm{T}_{\mathrm{E}} \mathrm{X}$ 


\section{Annotated Content}

Recall: Dependent $T=T$ without the independence property.

$\S 0$ Introduction, pg.3

$\S 1$ Expanding by making a type definable, p.4

[Suppose we expand $M \prec \mathfrak{C}$ by a relation for each set of the form $\{\bar{b}: \bar{b} \in$ ${ }^{m} M$ and $\left.\models \varphi[\bar{b}, \bar{a}]\right\}$, where $\bar{a} \in{ }^{\omega>} \mathfrak{C}, \varphi(\bar{x}, \bar{y}) \in \mathbb{L}\left(\tau_{T}\right)$ and $m=\ell g(\bar{x})$. We prove that the theory of this model is dependent and has elimination of quantifiers.]

$\S 2$ More on indiscernible sequences, p.15

[This is complimentary to [Sh 715, §5]. Dedekind cuts with cofinality from both sides $\leq \kappa+|T|=\kappa$ inside $\kappa$-saturated models (of a dependent theory $T)$ tend to be filled together.]

$\S 3$ Strongly dependent theories, p.26

[Being strongly dependent is related to being superstable; however, strongly dependent theories which are stable (called strongly stable) are not necessarily superstable. We start the investigation of this class of first order theories. In particular, for such a theory there is no non-algebraic types $p, q$ with definable functions essentially from $q(\mathfrak{C})$ onto ${ }^{\omega}(p(\mathfrak{C}))$. Also there is no equivalence relation on $p(\bar{x})$ with infinitely many equivalence classes, each class has essentially one to one definable correspondence with the whole.]

$\S 4$ Definable groups, p.31

[We start to investigate definable groups for dependent and strongly dependent theories, in particular with the size of the commutator of most members.]

$\S 5$ Non-forking, p.39-56

[We try to see what does non-forking satisfy for dependent theories.] 


\section{$\S 0$ INTRODUCTION}

The work in [Sh 715] tries to deal with the investigation of a (first order complete) theories $T$ which has the dependence property, i.e., does not have the independence property.

If $T$ is stable, expanding a model $M$ of $T$ by $p \uparrow \varphi(\bar{x}, \bar{y})$ for $p \in \mathbf{S}^{m}(M)$, that is expanding $M$ by the relation $R_{p, M}^{\varphi(\bar{x}, \bar{y})}=\left\{\bar{a} \in{ }^{\ell(\bar{y})} M: \varphi(\bar{x}, \bar{a}) \in p\right\}$ is an inessential one, i.e., by a relation on $M$ definable in $M$ with parameters. This fails for unstable theories but in $\S 1$ we prove a weak relative: if $T$ is a dependent theory then so is the expansion above, i.e., $\operatorname{Th}\left(M, R_{p, M}^{\varphi(\bar{x}, \bar{y})}\right)_{p, \varphi(\bar{x}, \bar{y})}$.

In [Sh 715, §5] it is shown that for any model $N$ of a dependent unstable $T$, we can find a $\kappa$-saturated model $M$ extending $N$ such that the following set is quite arbitrary: pairs of cofinalities of a cut in $M$ for some definable partial order in $N$ (so not fulfilled in $M$ ) or even the set of pairs $\left(\kappa_{1}, \kappa_{2}\right)$ of regular cardinals for which there is an indiscernible sequence $\left\langle a_{\alpha}: \alpha<\kappa_{1}\right\rangle-\left\langle b_{\beta}: \beta<\kappa_{2}^{*}\right\rangle$ such that the $\left(\kappa_{1}, \kappa_{2}\right)$-cut is respected in $M$, that is, we cannot find an element in $M$ which we can add after the $a_{\alpha}$ 's but before the $b_{\beta}$ 's linearly ordered by some $\varphi(x, y ; \bar{c})$ which is a partial order. However, there were restrictions on the cofinalities being not too small. In section 2 we show that, to a large extent, yes these restrictions are necessary.

The family of dependent theories is parallel to the family of stable theories. But actually better in some sense is the family of superstable, i.e. the balance of the "size" of the family of such theories and what we can tell about them seem better for the family of superstable ones. In $\S 3$ a related family, in a sense parallel to superstable, called strongly dependent theories, is defined. Now every superstable $T$ is strongly stable (defined as stable, strongly dependent), but the inverse fails (see also [Sh 839], [Sh:F660]). We then observe some basic properties. This is continued in [Sh 863].

In $\S 4$ we look at groups definable in models of dependent theories, and also in strongly dependent theories. In $\S 5$ we try to look systematically at a parallel to non-forking.

This work is continued in [Sh 876], [Sh 863], [Sh 886], [FiSh:E50], [CoSh:919], [Sh:F705], [Sh 877], [Sh:906], [Sh:900]. More specifically on a parallel to unidimensional for the theory of the real field see work of E. Firstenberg and S. Shelah [FiSh:E50]. Concerning strongly dependent theories (see section 3) we try to investigate them in [Sh 863].

We should add to the history in [Sh 715] that Keisler [Ke87] connects dependent theories and measures on the set of definable subsets of a model. Also Poizat [Po81] (and then [Sh:93, pg.202,3] answering positively a question of Poizat). Poizat dealing with the number of complete types in $\mathbf{S}(N)$ finitely satisfied in $M \prec N$; prove 
that the number is $\leq 2^{\{M \|}$ (when $|T| \leq\|M\|$ ) and his question was whether it is $\leq\left(\operatorname{Ded}\left(\|M\|^{|T|}\right)\right.$ was answered positively in [Sh:93]. Here in 5.26 we follow [Sh:93] proving that we can replace finitely satisfiable but does not split. Also [Sh 715, 3.2] is 5.2 of Baldwin-Benedikt [ $\backslash \mathrm{Ba} 1 \mathrm{~B} 100]$.

Note that Baisalov and Poizat [BaPo98] proved that if $T$ is o-minimal a theorem which is a consequence of $\S 1$.

We thank Eyal Firstenberg, Aviv Tatarsky and the referee for many helpful corrections and lately Itay Kaplan and friends for pointing our deficiencies in $§ 5$.

Notation:

As in [Sh 715] and, in addition

0.1 Definition. 1) For $\overline{\mathbf{b}}=\left\langle\bar{b}_{t}: t \in I\right\rangle$ an infinite indiscernible sequence, let $\operatorname{tp}^{\prime}(\overline{\mathbf{b}})=\left\langle\operatorname{tp}\left(\bar{b}_{t_{0}^{n}}{ }^{\wedge} \ldots{ }^{\wedge} \bar{b}_{t_{n-1}^{n}}, \emptyset, \mathfrak{C}\right): n<\omega\right\rangle$ where $t_{\ell}^{n}<_{I} t_{\ell, k}^{n}$ for $\ell<k<n<\omega$; the choice of the $t_{\ell}^{n}$ 's is immaterial.

2) Let " $M$ is $n$-saturated" mean " $M$ is $\aleph_{0}$-saturated" for $n<\omega$.

3) Let $A / B$ mean $\operatorname{tp}(A, B)$, inside $\mathfrak{C}$ or $\mathfrak{C}^{\mathrm{eq}}$, of course. 


\section{$\S 1$ EXPANDing BY MAKING A TYPE DEFINABLE}

What, in short, do we show here? We say $A$ is full over $M$ if every $p \in \mathbf{S}^{<\omega}(M)$ is realized in $A$, (Definition 1.5). We let $\mathfrak{B}_{A, M}$ be the expansion of $M$, for each $\varphi(\bar{x}, \bar{a}), \bar{a} \in{ }^{\omega>} A$, by the following $\ell g(\bar{x})$-place relation: all realizations of $\varphi(-, \bar{a})$, i.e., by $\varphi(M, \bar{a})$ (see Definition 1.10(2)). We prove here that if $A$ is full over $M$ then $\operatorname{Th}\left(\mathfrak{B}_{M, A}\right)$ has elimination of quantifiers (see Claim 1.12(1), its proof depends only on $1.2,1.7(2))$. By this we prove that $\operatorname{Th}\left(\mathfrak{B}_{M, A}\right)$ is dependent (in 1.13 depending on 1.10(4), 1.11(1),(5) only), so for this conclusion " $A$ is full over $M$ " is not needed.

1.1 Context. 1) $T$ is a (first order complete) dependent theory in the language $\mathbb{L}\left(\tau_{T}\right)$.

2) $\mathfrak{C}=\mathfrak{C}_{T}$ is a monster model for $T$.

1.2 Claim. Assume

(a) $M$ a model

(b) $D$ an ultrafilter on $M$, i.e. on the Boolean Algebra $\mathscr{P}(M)$.

Then for any $\bar{c} \in \omega>\mathfrak{C}$ and formula $\varphi(x, y, \bar{c})$ we have: if the set $\{a \in M:(\exists y \in$ $M)(\mathfrak{C} \models \varphi[a, y, \bar{c}])\}$ belongs to $D$ then it belongs to $\operatorname{def}_{2}(D)$, see definition below.

1.3 Definition. 1) When $D$ is an ultrafilter on a set $B \subseteq \mathfrak{C}$ let $\operatorname{def}_{2}(D)=\{A \in D$ : some member of $\operatorname{def}_{1}(D)$ is included in $\left.A\right\}$ where $\operatorname{def}_{1}(D)=\{A \in D$ : for some $\bar{c} \in{ }^{\omega>} \mathfrak{C}$ and formula $\psi(x, \bar{c})$ the set $\psi(M, \bar{c})=\{a \in M: \mathfrak{C} \models \psi(a, \bar{c})\}$ belongs to $D$ and is equal to $A$ \}.

2) Similarly when $D$ is an ultrafilter on ${ }^{m} B, m<\omega$.

1.4 Remark. Note the following easy comments.

1) Of course, Claim 1.2 holds also for $\varphi=\varphi(\bar{x}, \bar{y}, \bar{c})$ when $D$ an ultrafilter on ${ }^{m} M$ and $m=\ell g(\bar{y})$ because, e.g. we can just work in $\mathfrak{C}^{\mathrm{eq}}$.

2) $T$ is dependent iff $T^{\text {eq }}=\operatorname{Th}\left(\mathfrak{C}^{\mathrm{eq}}\right)$ is so (1) is dependent this justify the statement above in part (1) and $\operatorname{Th}(\mathfrak{C})$ is dependent iff $\operatorname{Th}(\mathfrak{C}, c)_{c \in C}$ is (for any $C \subseteq \mathfrak{C}$ ) and $T$ dependent $\Rightarrow \operatorname{Th}\left(\mathfrak{C} \uparrow \tau^{\prime}\right)$ is dependent when $\tau^{\prime} \subseteq \tau_{T}$.

3) Note that $\operatorname{def}_{1}(D)$ is a filter on $A$.

4) In the proof of 1.2 the hypothesis " $T$ dependent" is used only for deducing " $\varphi(x, y, \bar{c})$ is dependent" which is naturally defined.

5) Recall the following (which is used in the proof): 
(a) $\Delta \subseteq \mathbb{L}\left(\tau_{T}\right)$ means $\Delta$ is a set of objects of the form $\varphi(\bar{x}), \varphi$ a (first order) formula from $\mathbb{L}\left(\tau_{T}\right), \bar{x}$ a sequence of variables with no repetitions including the free variables, but changing the variables of $\varphi$ is allowed here, i.e., there is no difference between $\varphi(x)$ and $\varphi(y)$; we may write $\varphi(\bar{x}, \bar{y})$ instead of $\varphi\left(\bar{x}^{\wedge} \bar{y}\right)$

(b) $\operatorname{tp}_{\Delta}(\bar{a}, A)=\left\{\varphi(\bar{x}, \bar{b}): \bar{x}=\left\langle x_{\ell}: \ell<\ell g(\bar{a})\right\rangle, \varphi(\bar{x}, \bar{y}) \in \Delta\right.$ and $\mathfrak{C} \models \varphi[\bar{a}, \bar{b}]$ and $\left.b \in{ }^{\omega>} A\right\}$

(c) $\left\langle\bar{b}_{t}: t \in I\right\rangle$ is $\Delta$-indiscernible over $B$ means that $I$ is a linear order and if $\varphi\left(\bar{x}_{1}, \ldots, \bar{x}_{n}, \bar{y}\right) \in \Delta, \ell g\left(\bar{x}_{\ell}\right)=\ell g\left(\bar{b}_{t}\right)$ for $\ell=1, \ldots, n$ and $t \in I$ and $\bar{c} \in{ }^{\ell g(\bar{y})} B$ then for any $s_{1}<_{I} \ldots<_{I} s_{n}$ and $t_{1}<_{I} \ldots<_{I} t_{n}$ we have $\mathfrak{C} \models " \varphi\left[\bar{b}_{t_{1}}, \ldots, \bar{b}_{t_{n}}, \bar{c}\right] \equiv \varphi\left[\bar{b}_{s_{1}}, \ldots, \bar{b}_{s_{n}}, \bar{c}\right] "$.

6) In the proof of Claim 1.2 we do not need to close $\Delta_{1}$ to $\Delta_{2}$, i.e. we can let $\Delta_{2}=\Delta_{1}$ provided that we redefine $\operatorname{tp}_{\Delta_{1}}(a, A)$ as

$$
\begin{array}{r}
\operatorname{tp}(a, A) \cap\left\{\varphi\left(a_{0}, \ldots, a_{m-1}, x, a_{m+1}, \ldots, a_{n}\right): \varphi\left(x_{0}, \ldots, x_{m-1}, x_{m},\right.\right. \\
\left.\left.x_{m+1}, \ldots, x_{n-1}\right) \in \Delta\right\}
\end{array}
$$

or more specifically, in $(*)_{1}$ from $\bigotimes_{1}$, inside the proof of $\otimes_{1}$, we replace " $a_{\ell}$ realizes $\operatorname{tp}_{\Delta_{2}}\left(a_{w}, \ldots\right)$ " by " $a_{\ell}$ realizes $\left\{\varphi\left(a_{\ell_{0}}, \ldots, a_{\ell_{m-1}}, x, a_{\omega+1}, \ldots, a_{\omega+n-1+m}, \bar{b}\right)\right.$ : $\varphi\left(x_{0}, \ldots, x_{n-1}, \bar{y}\right) \in \Delta_{1}$ and $\bar{b} \in \ell g(\bar{y}) B, m<n, \ell_{0}<\ldots<\ell_{m-1}<\ell$ and $\left.\mathfrak{C} \models \varphi\left[a_{\ell_{0}}, \ldots, a_{\ell_{m-1}}, a_{\omega}, a_{\omega+n+1}, \ldots, a_{\omega+n-1-m}, \bar{b}\right)\right\}$.

Proof. We shall use " $T$ is dependent" only in the last sentence of the proof toward contradiction. Assume that $\bar{c}, \varphi(x, y, \bar{c})$ form a counterexample.

So

$\circledast_{0}(i) \quad$ the set $A^{*}=\{a \in M$ : for some $b \in M$ we have $\models \varphi[a, b, \bar{c}]\}$ belongs to $D$,

(ii) $\quad A^{*} \notin \operatorname{def}_{2}(D)$, that is, no $A^{\prime} \in \operatorname{def}_{1}(D)$ is included in $A^{*}$.

By the choice of $A^{*}$ we can, for each $a \in A^{*}$, choose $b_{a} \in M$ such that $\models \varphi\left[a, b_{a}, \bar{c}\right]$. Let $D_{1}=D$ and let $D_{2}$ be the following ultrafilter on ${ }^{2} M: X \in D_{2}$ iff $X \subseteq{ }^{2} M$ and for some $A \in D$ we have $\left\{\left(a, b_{a}\right): a \in A \cap A^{*}\right\} \subseteq X$.

We can choose $\left\langle\left(a_{\omega+n}, b_{\omega+n}\right): n<\omega\right\rangle$ from $\mathfrak{C}$ such that

$\circledast_{1}$ for $n_{1}<n_{2}<\omega$ the pair $\left(a_{\omega+n_{1}}, b_{\omega+n_{1}}\right)$ realizes the type $\operatorname{Av}\left(M \cup\left\{a_{\omega+\ell}, b_{\omega+\ell}\right.\right.$ : $\left.\left.\ell \in\left(n_{1}, n_{2}\right]\right\}, D_{2}\right)$. 
It follows that $a_{\omega+n_{1}}$ realizes the type $\operatorname{Av}\left(M \cup\left\{a_{\omega+\ell}, b_{\omega+\ell}: \ell \in\left(n_{1}, n_{2}\right]\right\}, D_{1}\right)$ and

$\circledast_{2}$ for $n_{1}<n_{2}$, the element $a_{\omega+n_{1}}$ realizes the type $\operatorname{Av}\left(M \cup\left\{a_{\omega+\ell}: \ell \in\right.\right.$ $\left.\left.\left(n_{1}, n_{2}\right]\right\}, D\right)$

$\circledast_{3}$ for $n_{1}<n_{2}$ the triple $\left(a_{2 n_{1}}, a_{2 n_{1}+1}, b_{2 n_{1}+1}\right)$ realizes the type $\operatorname{Av}(M \cup$ $\left.\left\{a_{\omega+2 \ell}, a_{\omega+2 \ell+1}, b_{\omega+2 \ell+1}: \ell \in\left(n_{1}, n_{2}\right]\right\}, D_{3}\right)$ for some ultrafilter $D_{3}$ on $M^{3}$. [Why? We define $D_{3}:=\left\{X \subseteq M^{3}:\{a \in M:\{(b, c) \in M \times M:(a, b, c) \in\right.$ $\left.\left.X\} \in D_{2}\right\} \in D_{1}\right\}$.]

(we use mainly $\circledast_{1}$ ).

Now clearly

$\bigotimes_{0}\left\langle\left(a_{\omega+n}, b_{\omega+n}\right): n\langle\omega\rangle\right.$ is an indiscernible sequence over $M$

$\otimes_{1}$ if $\Delta_{1} \subseteq \mathbb{L}\left(\tau_{T}\right)$ is finite then we can find $n(*)<\omega$ and finite $\Delta_{2} \subseteq \mathbb{L}(T)$ such that

$(*)_{1}$ if $n_{1}<\omega$ and $B \subseteq M$ is finite and for each $\ell<n_{1}$ the element $a_{\ell} \in M$ realizes the type $\operatorname{tp}_{\Delta_{2}}\left(a_{\omega},\left\{a_{0}, \ldots, a_{\ell-1}\right\} \cup\left\{a_{\omega+1}, \ldots, a_{\omega+n(*)}\right\} \cup B\right)$ then $\left\langle a_{\ell}: \ell<\right.$ $\left.n_{1}\right\rangle^{\wedge}\left\langle a_{\omega+\ell}: \ell<\omega\right\rangle$ is a $\Delta_{1}$-indiscernible sequence over $B$ (and even $\Delta_{2}$-indiscernible).

Note that this is close to [Sh $715,1.16=\mathrm{np} 1.5 \mathrm{tex}]$; note that it follows from the result that even for $n_{1}=\omega$ this holds.

[Why does $\nabla_{1}$ hold? Let $n(*)$ be $\operatorname{arity}\left(\Delta_{1}\right)$, i.e., the maximal number of free variables of a formula from $\Delta_{1}$, it is finite as $\Delta_{1}$ is finite so without loss of generality each $\varphi \in \Delta_{1}$ is $\varphi(\bar{x})$, $\operatorname{Rang}(\bar{x}) \subseteq\left\{x_{\ell}: \ell<n(*)\right\}$. Let $\Delta_{2}$ be the closure of $\Delta_{1}$ under identifying and permuting the variables and let $\Delta_{2, k}$ be defined like $\Delta_{2}$ but we allow to add from $\left\{x_{0}, \ldots, x_{k}\right\}$ dummy variables to each formula (we can use below $\left.\Delta_{2}=\cup\left\{\Delta_{2, k}: k<\omega\right\}\right)$. We have to prove that for this choice of $n(*)$ and $\Delta_{2}$ the assertion $(*)_{1}$ holds.

So assume $n_{1}<\omega$ and $B, a_{\ell}$ (for $\ell<n_{1}$ ) are as required in the assumption of $(*)_{1}$. Now we prove by induction on $k \leq n_{1}$ that

$(*)_{k}^{1}$ the sequences $\left\langle a_{\omega+\ell}: \ell<n_{1}+n(*)\right\rangle$ and $\left\langle a_{\ell}: \ell<k\right\rangle^{\wedge}\left\langle a_{\omega+\ell}: \ell<n_{1}+\right.$ $n(*)-k\rangle$ realize the same $\Delta_{2, n_{1}+n(*)}$-type over $B$ which means that: if $m \leq$ $n(*), \bar{d} \in{ }^{m} B$ and $\varphi\left(\bar{y}_{1}, \bar{y}_{2}\right) \in \Delta_{2, n_{1}+n(*)+m}, \ell g\left(\bar{y}_{1}\right)=n_{1}+n(*), \ell g\left(\bar{y}_{2}\right)=m$ then $\mathfrak{C} \models \varphi\left[\left\langle a_{\omega+\ell}: \ell<n_{1}+n(*)\right\rangle, \bar{d}\right]$ iff $\mathfrak{C} \models \varphi\left[\left\langle a_{\ell}: \ell<k\right\rangle^{\wedge}\left\langle a_{\omega+\ell}: \ell<\right.\right.$ $\left.\left.n_{1}+n(*)-k\right\rangle, \bar{d}\right]$; note that we can allow $m \leq n(*)$.

For $k=0$, the two expressions gives the same sequence. Assume this holds for $k$ and we shall prove it for $k+1$. First $\left\langle a_{\ell}: \ell<k+1\right\rangle^{\wedge}\left\langle a_{\omega+\ell}: \ell<n_{1}+n(*)-(k+1)\right\rangle$ realize the same type as $\left\langle a_{0}, \ldots, a_{k}, a_{\omega+1}, \ldots, a_{\omega+n_{1}+n(*)-(k+1)}\right\rangle$ simply as $\left\langle a_{\omega+\ell}\right.$ : 
$\ell<\omega\rangle$ is an indiscernible sequence over $M$, by $\otimes_{0}$. Now by the assumption of $(*)_{1}$ we know that $a_{k}, a_{\omega}$ realizes the same $\Delta_{2}$-type over $B \cup\left\{a_{0}, \ldots, a_{k-1}\right\} \cup$ $\left\{a_{\omega+1}, \ldots, a_{\omega+n_{1}+n(*)-k}\right\}$.

As $n(*)$ is the arity of $\Delta_{1}$ hence also of $\Delta_{2}$ and the definition of $\Delta_{2, n_{1}+n(*)}$ it follows that the sequence $\left\langle a_{0}, \ldots, a_{k-1}, a_{k}, a_{\omega+1}, \ldots, a_{\omega+n_{1}+n(*)-k-1}\right\rangle$ realizes over $B$ the same $\Delta_{2, n_{1}+n(*)}$-type as the sequence

$\left\langle a_{0}, \ldots, a_{k-1}, a_{\omega}, a_{\omega+1}, \ldots, a_{\omega+n_{1}+n(*)-k-1}\right\rangle$ but by the induction hypothesis on $k$ the latter realizes over $B$ the same $\Delta_{2, n_{1}+n(*)}$-type as the sequence $\left\langle a_{\omega}, a_{\omega+1}, \ldots, a_{\omega+n_{1}+n(*)-1}\right\rangle$, hence $(*)_{k+1}^{1}$ holds so we have carried the induction on $k \leq n_{1}$. Now the desired conclusion follows from $(*)_{m}^{1}$ by $\otimes_{0}$ as each formula in $\Delta_{1}$ and even $\Delta_{2}$ has $\leq n(*)$ free variables.]

$\bigotimes_{2}$ if $\Delta_{1} \subseteq \mathbb{L}\left(\tau_{T}\right)$ is finite $\underline{\text { then }}$ we can find $n(*)<\omega$ and finite $\Delta_{2} \subseteq \mathbb{L}\left(\tau_{T}\right)$ such that

$(*)_{2}$ if $n_{1}<\omega, B \subseteq M$ is finite and for each $\ell<n_{1}, a_{2 \ell} \in M$ realizes $\operatorname{tp}_{\Delta_{2}}\left(a_{\omega},\left\{a_{2 m}, a_{2 m+1}, b_{2 m+1}: m<\ell\right\} \cup\left\{a_{\omega+\ell}, b_{\omega+\ell}: \ell=1, \ldots, n(*)\right\} \cup\right.$ $B)$ and $\left\langle a_{2 \ell+1}, b_{2 \ell+1}\right\rangle$ realizes $\operatorname{tp}_{\Delta_{2}}\left(\left(a_{\omega}, b_{\omega}\right),\left\{a_{2 m}, a_{2 m+1}, b_{2 m+1}: m<\right.\right.$ $\left.\left.\left.\ell\} \cup\left\{a_{2 \ell}\right\} \cup\left\{a_{\omega+\ell}, b_{\omega+\ell}: \ell=1, \ldots, n(*)\right\} \cup B\right)\right\}\right)$ then $\left\langle\left(a_{2 \ell}, a_{2 \ell+1}, b_{2 \ell+1}\right):\right.$ $\left.\left.\ell<n_{1}\right\rangle^{\wedge}\left\langle a_{\omega+2 \ell}, a_{\omega+2 \ell+1}, b_{\omega+2 \ell+1}: \ell<\omega\right\rangle\right)$ is $\Delta_{1}$-indiscernible over $B$ (and even $\Delta_{2}$-indiscernible)

[Why? The proof is similar to the proof of $\bigotimes_{1}$ mainly replacing the use of $\circledast_{1}$ by $\circledast_{3}$.]

$\nabla_{3}$ if $B \subseteq M$ is finite, $n^{*}<\omega$ and $\Delta \subseteq \mathbb{L}\left(\tau_{T}\right)$ is finite, then we can find $a \in M$ realizing the finite type $q=\operatorname{tp}_{\Delta}\left(a_{\omega}, B \cup\left\{a_{\omega+\ell}, b_{\omega+\ell}: \ell=1, \ldots, n^{*}\right\}\right)$ such that $\models \neg(\exists y \in M) \varphi(a, y, \bar{c})$.

[Why? The set $A:=\{a \in M: a$ realizes $q$, equivalently satisfies the formula $\wedge q \in \operatorname{Av}(\mathfrak{C}, D)\}$ belongs to $D$ because $q$ is finite and the choice of $\left\langle a_{\omega+\ell}, b_{\omega+\ell}: \ell<\omega\right\rangle$; moreover, it belongs to $\operatorname{def}_{1}(D)$ by the definition of $\operatorname{def}_{1}(D)$ as $\wedge q$ is a formula. But $\operatorname{def}_{1}(D) \subseteq \operatorname{def}_{2}(D)$ hence $A \in \operatorname{def}_{2}(D)$.

So by the assumption toward contradiction and choice of $A^{*}$, i.e., by $(*)_{0}$, we have $\neg\left(A \subseteq A^{*}\right)$ so there is $a \in A$ such that $a \notin A^{*}$ which means that $\neg(\exists y \in M) \varphi(a, y, \bar{c})$, so we are done.]

By the above and compactness (or use an ultrapower)

$\bigotimes_{4}$ there are $N, a_{2 n}, a_{2 n+1}, b_{2 n+1}($ for $n<\omega)$ such that

(a) $N$ is $|T|^{+}$-saturated

(b) $a_{2 n}, a_{2 n+1}, b_{2 n+1} \in N$

(c) $\left\langle a_{n}: n<\omega\right\rangle$ is an indiscernible sequence

(d) $\left\langle\left(a_{2 n}, a_{2 n+1}, b_{2 n+1}\right): n<\omega\right\rangle^{\wedge}\left\langle\left(a_{\omega+2 n}, a_{\omega+2 n+1}, b_{\omega+2 n+1}\right): n<\omega\right\rangle$ is an indiscernible sequence 
(e) $\mathfrak{C} \models \varphi\left[a_{2 n+1}, b_{2 n+1}, \bar{c}\right]$

(f) for no $n<\omega$ and $b \in N$ do we have $\mathfrak{C} \models \varphi\left[a_{2 n}, b, \bar{c}\right]$.

[Why? By compactness it is enough to prove the following: for every $n_{1}<\omega$ and finite $\Delta_{1} \subseteq \mathbb{L}\left(\tau_{T}\right)$ to which $\varphi$ belongs there are $a_{2 n}, a_{2 n+1}, b_{2 n+1} \in M$ for $n<n_{1}$ such that clauses (a)-(f) holds when we restrict ourselves to $n<n_{1}$ and $\Delta_{1}$-types replacing $N$ by $M$. We first choose a finite $\Delta_{2} \subseteq \mathbb{L}\left(\tau_{T}\right)$ as in $\nabla_{2}$, and then choose $\left(a_{2 n}, a_{2 n+1}, b_{2 n+1}\right)$ by induction on $n$ such that the demand in $(*)_{2}$ of $\bigotimes_{2}$ holds. Arriving to $n$ choose $a_{2 n} \in M$ such that in addition, clause (f) holds, this is possible by $\otimes_{3}$, and then choose $\left(a_{2 n+1}, b_{2 n+1}\right) \in{ }^{2} M$ recalling $\left(a_{\omega}, b_{\omega}\right)$ realizes $\operatorname{Av}\left(M \cup\left\{a_{\omega+n}, b_{\omega+n}: 1 \leq n<\omega\right\}, D_{2}\right)$. So we are done proving $\bigotimes_{4}$.]

Next by clause (d) of $\otimes_{4}$

$\bigotimes_{5}$ there is an automorphism $F$ of $\mathfrak{C}$ such that

$n<\omega$ implies $F\left(\left(a_{\omega+2 n}, a_{\omega+2 n+1}, b_{\omega+2 n+1}\right)\right)=\left(a_{2 n}, a_{2 n+1}, b_{2 n+1}\right)$.

Hence we can find $b_{2 n} \in \mathfrak{C}$ for $n<\omega$ such that $\left\langle\left(a_{n}, b_{n}\right): n<\omega\right\rangle$ is an indiscernible sequence (over $\emptyset$, not necessarily over $\bar{c} !$ ) and as $N$ is $|T|^{+}$-saturated without loss of generality $b_{2 n} \in N$ for $n<\omega$. But $\mathfrak{C} \models \varphi\left[a_{2 n+1}, b_{2 n+1}, \bar{c}\right]$ for $n<\omega$ by clause (e) of $\nabla_{4}$ so as $T$ is dependent for every large enough $n<\omega$ we have $\mathfrak{C} \models \varphi\left[a_{2 n}, b_{2 n}, \bar{c}\right]$. But as $b_{2 n} \in N$ clearly $\left\{a_{n}, b_{n}: n<\omega\right\} \subseteq N$ hence $n<\omega \Rightarrow \mathfrak{C} \models \varphi\left[a_{2 n}, b_{2 n}, \bar{c}\right]$ contradicting clause (f) of $\bigotimes_{4}$.

Recall

1.5 Definition. For $A \subseteq C(\subseteq \mathfrak{C})$ we say that $C$ is full over $A$ when: for every $m<\omega$ and $p \in \mathbf{S}^{m}(A)$, there is $\bar{c} \in{ }^{m} C$ which realizes $p$.

1.6 Observation. We have $\operatorname{def}_{\ell}\left(D_{1}\right)=\operatorname{def}_{\ell}\left(D_{2}\right)$ for $\ell=1,2 \underline{\text { when: }}$

(a) $D_{1}, D_{2}$ are ultrafilters on ${ }^{m} A$,

(b) $A \subseteq C$,

(c) $C$ is full over $A$,

(d) $\operatorname{Av}\left(C, D_{1}\right)=\operatorname{Av}\left(C, D_{2}\right)$.

Proof. Easy. 
1.7 Claim. 1) Assume

(a) $M \subseteq C$

(b) $D_{0}$ is an ultrafilter on ${ }^{m_{0}} M$

(c) $\bar{b}_{0}$ realizes $\operatorname{Av}\left(C, D_{0}\right)$

(d) $\operatorname{tp}\left(\bar{b}_{0}{ }^{\wedge} \bar{b}_{1}, C\right)$ is f.s. in $M$ and $m_{1}=\ell g\left(\bar{b}_{1}\right)$

(e) $C$ is full over $M$.

Then for some ultrafilter $D$ on ${ }^{m_{0}+m_{1}} M$ we have

( $\alpha) \operatorname{Av}(C, D)=\operatorname{tp}\left(\bar{b}_{0}{ }^{\wedge} \bar{b}_{1}, C\right)$

$(\beta)$ the projection of $D$ on ${ }^{m_{0}} M$ is $D_{0}$.

2) Assume that clauses (a) and (e) of part (1) hold. Then for any $\bar{c} \in \omega>\mathfrak{C}$ and formula $\varphi(\bar{x}, y, \bar{z}) \in \mathbb{L}\left(\tau_{T}\right), \ell g(\bar{z})=\ell g(\bar{c})$ there are $\psi\left(\bar{x}, \overline{\bar{z}^{\prime}}\right) \in \mathbb{L}\left(\tau_{T}\right)$ and $\bar{d}$ of length $\ell g\left(\bar{z}^{\prime}\right)$ from $\mathfrak{C}$, (and even from $C$ ) such that $\{\bar{a} \in M:(\exists y \in M)(\mathfrak{C} \models \varphi[\bar{a}, y, \bar{c}])\}=$ $\{\bar{a} \in M: \mathfrak{C} \models \psi(\bar{a}, \bar{d})\}$.

Proof. 1) Let

$$
\begin{aligned}
& \mathscr{E}_{0}=\left\{\left\{\bar{a} \in{ }^{m_{0}+m_{1}} M: \bar{a} \uparrow m_{0} \in X\right\}: X \in D_{0}\right\} \\
& \mathscr{E}_{1}=\left\{\left\{\bar{a} \in{ }^{m_{0}+m_{1}} M: \mathfrak{C} \models \varphi[\bar{a} ; \bar{c}]\right\}: \varphi(\bar{x} ; \bar{y}) \in \mathbb{L}\left(\tau_{T}\right)\right. \\
& \ell g(\bar{x})=m_{0}+m_{1}, \ell g(\bar{y})=\ell g(\bar{c}), \\
& \left.\bar{c} \in{ }^{\omega>} C \text { and } \mathfrak{C} \models \varphi\left[\bar{b}_{0} \frown \bar{b}_{1} ; \bar{c}\right]\right\} \text {. }
\end{aligned}
$$

Clearly it suffices to prove that there is an ultrafilter on ${ }^{m_{0}+m_{1}} M$ extending $\mathscr{E}_{0} \cup \mathscr{E}_{1}$. For this it suffices to show that any finite subfamily of $\mathscr{E}_{0} \cup \mathscr{E}_{1}$ has a non-empty intersection. But $\mathscr{E}_{0}$ is closed under finite intersections as $D_{0}$ is an ultrafilter on ${ }^{m_{0}} M$ and $\mathscr{E}_{1}$ is closed under finite intersections as $\mathbb{L}\left(\tau_{T}\right)$ is closed under conjunctions, so it suffices to prove that $X_{0} \cap X_{1} \neq \emptyset$ when

(i) $X_{0}=\left\{\bar{a} \in m_{0}+m_{1} M: \bar{a} \uparrow m_{0} \in X\right\} \in \mathscr{E}_{0}$ for some $X \in D_{0}$

(ii) $X_{1}=\left\{\bar{a} \in m_{0}+m_{1} M: \mathfrak{C} \models \varphi\left[\bar{b}_{0}{ }^{\wedge} \bar{b}_{1} ; \bar{c}\right]\right\} \in \mathscr{E}_{1}$ where $\varphi(\bar{x}, \bar{y})$ and $\bar{c}$ are as in the definition of $\mathscr{E} 1$. 
As $\operatorname{tp}\left(\bar{b}_{0}{ }^{\wedge} \bar{b}_{1}, C\right)$ is finitely satisfiable in $M(=$ assumption $(d))$ clearly there is an ultrafilter $D_{1}^{\prime}$ on ${ }^{m_{0}+m_{1}} M$ such that $\operatorname{Av}\left(C, D_{1}^{\prime}\right)=\operatorname{tp}\left(\bar{b}_{0}{ }^{\wedge} \bar{b}_{1}, C\right)$.

Let $D_{0}^{\prime}$ be the projection of $D_{1}^{\prime}$ to ${ }^{m_{0}} M$, i.e., $\left\{Y \subseteq{ }^{m_{0}} M:\left\{\bar{a} \in{ }^{m_{0}+m_{1}} M: \bar{a} \uparrow\right.\right.$ $\left.\left.m_{0} \in Y\right\} \in D_{1}^{\prime}\right\}$. Clearly $D_{0}^{\prime}$ is an ultrafilter over $m_{0} M$. We have $\mathfrak{C} \models \varphi\left[\bar{b}_{0}, \bar{b}_{1} ; \bar{c}\right]$, so $X_{1} \in D_{1}^{\prime}$ hence $X_{0}^{\prime}=\left\{\bar{a} \uparrow m_{0}: \bar{a} \in X_{1}\right\} \in D_{0}^{\prime}$; which implies that the set $X_{0}^{\prime \prime}:=\left\{\bar{a}_{0} \in{ }^{m_{0}} M:\right.$ for some $\bar{a}_{1} \in{ }^{m_{1}} M$ we have $\bar{a}_{0}{ }^{\wedge} \bar{a}_{1} \in X_{1}$, i.e., $\left.\models \varphi\left[\bar{a}_{0}, \bar{a}_{1} ; \bar{c}\right]\right\}$ belongs to $D_{0}^{\prime}$.

By 1.2 (and 1.4(1),(2)) it follows that $X_{0}^{\prime \prime}$ includes some $Y_{0}^{\prime \prime} \in \operatorname{def}_{1}\left(D_{0}^{\prime}\right)$. Now $\operatorname{Av}\left(C, D_{0}\right)=\operatorname{tp}\left(\bar{b}_{0}, C\right)=\operatorname{Av}\left(C, D_{0}^{\prime}\right)$, because the first equality holds as by assumption (b) the sequence $\bar{b}_{0}$ realizes $\operatorname{Av}\left(C, D_{0}\right)$ and second equality holds as $\bar{b}_{0}{ }^{\wedge} \bar{b}_{1}$ realizes $\operatorname{Av}\left(C, D_{1}^{\prime}\right)$ and the choice of $D_{0}^{\prime}$. But by assumption (e) every $p \in \mathbf{S}^{<\omega}(M)$ is realized by some sequence from $C$, hence by Observation 1.6 we have $\operatorname{def}_{2}\left(D_{0}\right)=\operatorname{def}_{2}\left(D_{0}^{\prime}\right)$. But $Y_{0}^{\prime \prime} \in \operatorname{def}_{1}\left(D_{0}^{\prime}\right)$ so $Y_{0}^{\prime \prime} \in \operatorname{def}_{2}\left(D_{0}\right)$ hence $Y_{0}^{\prime \prime} \in D_{0}$. By the choice of $Y_{0}^{\prime \prime}$ we have $Y_{0}^{\prime \prime} \subseteq X_{0}^{\prime \prime} \subseteq m_{0} M$ so by the previous sentence $X_{0}^{\prime \prime} \in D_{0}$, but by clause (i) above also $X \in D_{0}$ hence $X \cap X_{0}^{\prime \prime} \in D_{0}$, so we can find $\bar{a}_{0} \in X \cap X_{0}^{\prime \prime} \subseteq{ }^{m_{0}} M$. By the definition of $X_{0}^{\prime \prime}$ there is $\bar{a}_{1} \in{ }^{m_{1}} M$ such that $\mathfrak{C} \models \varphi\left[\bar{a}_{0}, \bar{a}_{1} ; \bar{c}\right]$. Now $\bar{a}_{0}{ }^{\wedge} \bar{a}_{1} \in X_{1}$ by the definition of $X_{1}$ from clause (ii) and $\bar{a}_{0} \wedge \bar{a}_{1} \in X_{0}$ because $\bar{a}_{0} \in X$ and $X_{0}$ 's definition from clause $(i)$. So $\bar{a}_{0}{ }^{\wedge} \bar{a}_{1} \in X_{0} \cap X_{1}$ hence $X_{0} \cap X_{1} \neq \emptyset$ and we are done.

2) Let $\varphi^{*}(\bar{x}, y, \bar{z}) \in \mathbb{L}\left(\tau_{T}\right)$ and $\bar{c}^{*} \in \in^{\ell g(\bar{z})} \mathfrak{C}$ and we should find $\psi\left(\bar{x}, \bar{z}^{\prime}\right), \bar{d}$ as required. Let $\bar{c} \in{ }^{\ell g(\bar{z})} C$ realizes $\operatorname{tp}\left(\bar{c}^{*}, M\right)$ for our purpose we may assume without loss of generality $\bar{c}^{*}=$ $\bar{c}$. For any formula $\psi\left(\bar{x}, \bar{z}^{\prime}\right) \in \mathbb{L}\left(\tau_{T}\right)$ and $\bar{d} \in{ }^{\ell g\left(\bar{z}^{\prime}\right)} \mathfrak{C}$ let $Y_{\psi(\bar{x}, \bar{d}), M}=\left\{\bar{a} \in{ }^{\ell g(\bar{x})} M\right.$ : $\mathfrak{C} \models \psi[\bar{a}, \bar{d}]\}$ and let $X_{\varphi(\bar{x}, y, \bar{c}), M}=\left\{\bar{a} \in{ }^{\ell(\bar{x})} M: \mathfrak{C} \models \varphi[\bar{a}, b, \bar{c}]\right.$ for some $\left.b \in M\right\}$.

Lastly, let $\mathscr{P}=\left\{Y_{\psi(\bar{x}, \bar{d}), M}: \psi(\bar{x}, \bar{y}) \in \mathbb{L}\left(\tau_{T}\right), \bar{d} \in{ }^{\ell g(\bar{z})} C\right.$ and $Y_{\psi(\bar{x}, \bar{d}), M} \subseteq$ $\left.X_{\varphi(\bar{x}, y, \bar{c}), M}\right\}$.

Clearly $\mathscr{P}$ is closed under finite unions and is a family of subsets of $M$. Also if $X_{\varphi(\bar{x}, y, \bar{c}), M}$ is equal to some member of $\mathscr{P}$ then we are done, so assume toward

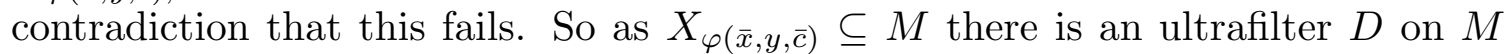
such that $X_{\varphi(\bar{x}, y, \bar{c}), M} \in D$ but $D$ is disjoint to $\mathscr{P}$ which contradicts $1.2 . \quad \square_{1.7}$

\subsection{Conclusion. Assume}

(a) $M \prec M_{1}$

(b) $M_{1}$ is $\|M\|^{+}$-saturated.

Then $\left\{A: A / M_{1}\right.$ is f.s. in $\left.M\right\}$ has amalgamation and JEP (the joint embedding property) by elementary maps from $\mathfrak{C}$ to $\mathfrak{C}$ which are the identity on $M_{1}$.

Proof. The joint embedding property is trivial. For the amalgamation, by compactness we should consider finite sequence $\bar{a}_{0}, \bar{a}_{1}, \bar{a}_{2}$ such that $\operatorname{tp}\left(\bar{a}_{0}{ }^{\wedge} \bar{a}_{\ell}, M_{1}\right)$ is f.s. 
in $M$ for $\ell=1,2$ and we should find sequences $\bar{b}_{0}, \bar{b}_{1}, \bar{b}_{2}$ such that $\ell g\left(\bar{b}_{\ell}\right)=\ell g\left(\bar{a}_{\ell}\right)$ for $\ell=0,1,2$ and $\operatorname{tp}\left(\bar{a}_{0}{ }^{\wedge} \bar{a}_{\ell}, M_{1}\right)=\operatorname{tp}\left(\bar{b}_{0}{ }^{\wedge} \bar{b}_{\ell}, M_{1}\right)$ for $\ell=1,2$ and $\operatorname{tp}\left(\bar{b}_{0}{ }^{\wedge} \bar{b}_{1}{ }^{\wedge} \bar{b}_{2}, M_{1}\right)$ is f.s. in $M$.

Let $m_{\ell}=\ell g\left(a_{\ell}\right)$, let $D_{0}$ be an ultrafilter on ${ }^{m_{0}} M$ such that $\operatorname{tp}\left(\bar{a}_{0}, M_{1}\right)=\operatorname{Av}\left(M_{1}, D_{0}\right)$. By 1.7(1) for $\ell \in\{1,2\}$ there is an ultrafilter $D_{\ell}$ on ${ }^{m_{0}+m_{\ell}} M$ such that

$(*)_{1} \operatorname{tp}\left(\bar{a}_{0} \hat{a}_{\ell}, M_{1}\right)$ is $\operatorname{Av}\left(M_{1}, D_{\ell}\right)$

$(*)_{2}$ the projection of $D_{\ell}$ on ${ }^{m_{0}} M$ is $D_{0}$.

Let $m=m_{0}+m_{1}+m_{2}$ and let $D_{1}^{\prime}$ be the filter on ${ }^{m} M$ consisting of $\left\{Y \subseteq{ }^{m} M\right.$ : for some $X \in D_{1}$ for every $\bar{a} \in{ }^{m} M$ we have $\left.\bar{a} \uparrow\left(m_{0}+m_{1}\right) \in X \Rightarrow \bar{a} \in Y\right\}$ and let $D_{2}^{\prime}$ be the filter on ${ }^{m} M$ consisting of $\left\{Y \subseteq{ }^{m} M\right.$ : for some $X \in D_{2}$ for every $\bar{a} \in{ }^{m} M$ we have $\left.\left(\bar{a} \uparrow m_{0}\right)^{\wedge}\left(\bar{a} \uparrow\left[m_{0}+m_{1}, m\right)\right) \in X \Rightarrow \bar{a} \in Y\right\}$. Easily $Y_{1} \in D_{1}^{\prime} \quad \& \quad Y_{2} \in D_{2}^{\prime} \Rightarrow Y_{1} \cap Y_{2} \neq \emptyset$ because $D_{1}, D_{2}$ has the same projection on $m_{0} M$.

Hence we can find an ultrafilter $D^{*}$ on ${ }^{m_{0}+m_{1}+m_{2}} M$ which extends $D_{1}^{\prime} \cup D_{2}^{\prime}$ hence if $\bar{b}_{0}{ }^{\wedge} \bar{b}_{1}{ }^{\wedge} \bar{b}_{2}$ realizes $\operatorname{Av}\left(M_{1}, D^{*}\right)$ then $\bar{b}_{0}{ }^{\wedge} \bar{b}_{\ell}$ realizes $\operatorname{tp}\left(\bar{a}_{0}{ }^{\wedge} \bar{a}_{\ell}, M_{1}\right)$ for $\ell=1,2$. So we are done.

1.9 Discussion: Next we shall deduce the promised results. If $M^{+}$is an expansion of a model $M \prec \mathfrak{C}$ by the restriction of relations definable in $\mathfrak{C}$ (with parameters) then $\operatorname{Th}\left(M^{+}\right)$is still dependent. Moreover, if we do this for close enough family of such relations then $\operatorname{Th}\left(M^{+}\right)$has elimination of quantifiers. Toward formulating this result we define several extensions of $T$.

1.10 Definition. Let $M \prec \mathfrak{C}, A \subseteq \mathfrak{C}$ and for simplicity $\tau_{T}$ has predicate symbols only.

1) We define a universal first order theory $T_{M, A}$ as follows

(a) the vocabulary is

$\tau_{M, A}=\left\{P_{\varphi(\bar{x}, \bar{a})}: \varphi \in \mathbb{L}\left(\tau_{T}\right)\right.$ and $\left.\bar{a} \in{ }^{\ell g(\bar{y})} A\right\} \cup\left\{c_{a}: a \in M\right\}$

with

(i) $c_{a}$ an individual constant

(ii) $P_{\varphi(\bar{x}, \bar{a})}$ being a predicate with arity $\ell g(\bar{x})$; but we identify $P_{R(\bar{x})}$ with $R$ (where $\left.\bar{x}=\left\langle x_{\ell}: \ell<\operatorname{arity}(R)\right\rangle\right)$ so $\tau_{T} \subseteq \tau_{M, A}$ )

(b) $T_{M, A}$ is the set of universal (first order) sentences satisfied in $\mathfrak{B}_{M, M, A}$, see part (2).

2) Assume $M \subseteq C \prec \mathfrak{C}$ and $\operatorname{tp}(C, M \cup A)$ is f.s. in $M$ (e.g., $C=M)$. We define $\mathfrak{B}=\mathfrak{B}_{C, M, A}$ as the $\tau_{M, A^{-}}$-model with universe $C$ such that $P_{\varphi(\bar{x}, \bar{a})}^{\mathfrak{B}}=\left\{\bar{b} \in{ }^{\ell g(\bar{x})} C\right.$ : 
$\mathfrak{C} \models \varphi[\bar{b}, \bar{a}]\}$ for $\varphi(\bar{x}, \bar{y}) \in \mathbb{L}\left(\tau_{T}\right), \bar{a} \in \in^{\ell(\bar{y})}(A)$ and such that $c_{a}^{\mathfrak{B}}=a$ for $a \in M$. If $C=M$ we may omit $C$.

3) A model $\mathfrak{B}$ of $T_{M, A}$ is called quasi-standard if $c_{a}^{\mathfrak{B}}=a$ for $a \in M$.

$3 \mathrm{~A}$ ) A model $\mathfrak{B}$ of $T_{M, A}$ is called standard if it is $\mathfrak{B}_{C, M, A}$ for some $C, M \subseteq C \subseteq \mathfrak{C}$ satisfying $\operatorname{tp}(C, M \cup A)$ is finitely satisfiable in $M$.

4) Let $T_{M, A}^{*}$ be the model completion of $T_{M, A}$ (well defined only if it exists!)

1.11 Observation. 1) If $M \subseteq C$ and $\operatorname{tp}(C, M \cup A)$ is finitely satisfiable in $M$, then $\mathfrak{B}_{C, M, A}$ is a model of $T_{M, A}$.

2) If $\mathfrak{B}$ is a model of $T_{M, A}$, then $\mathfrak{B}$ is isomorphic to the standard model $\mathfrak{B}=\mathfrak{B}_{C, M, A}$ of $T_{M, A}$ for some $C$.

3) Moreover, if $\mathfrak{B}_{1} \subseteq \mathfrak{B}_{2}$ are models of $T_{M, A}$ and $\mathfrak{B}_{1}$ is standard, then $\mathfrak{B}_{2}$ is (quasi standard and is) isomorphic over $\mathfrak{B}_{1}$ to some standard $\mathfrak{B}_{2}^{\prime}$ satisfying $\mathfrak{B}_{1} \subseteq \mathfrak{B}_{2}^{\prime}$.

4) If $A_{1} \subseteq A_{2}, M \subseteq C$ and $\operatorname{tp}\left(C, M \cup A_{2}\right)$ is f.s. in $M$ then $\mathfrak{B}_{C, M, A_{1}}$ is a reduct of $\mathfrak{B}_{C, M, A_{2}}$.

5) If $M \subseteq C_{1} \subseteq C_{2}$ and $\operatorname{tp}\left(C_{2}, M \cup A\right)$ is f.s. in $M$ then $\mathfrak{B}_{C_{1}, M, A}$ is a submodel of $\mathfrak{B}_{C_{2}, M, A}$ (and $\operatorname{tp}\left(C_{1}, M \cup A\right)$ is finitely satisfiable in $M$ hence $\mathfrak{B}_{C_{1}, M, A}$ is well defined).

Proof. Easy.

1.12 Claim. Assume $A$ is full over $M$.

1) $\mathfrak{B}_{M, M, A}$ is a model of $T_{M, A}$ with elimination of quantifiers; in fact every subset of $^{m}\left(\mathfrak{B}_{M, M, A}\right)$, i.e., of ${ }^{m}|M|$ definable in $\mathfrak{B}_{M, M, A}$ by some first order formula with parameters is definable by an atomic formula $R\left(x_{0}, \ldots, x_{m-1}\right)$ in this model.

2) If $\operatorname{tp}(C, A)$ is f.s. in $M$ then we can find $M^{+}$such that

(a) $M \cup C \subseteq M^{+} \prec \mathfrak{C}$

(b) $\operatorname{tp}\left(M^{+}, A\right)$ is f.s. in $M$

(c) $\mathfrak{B}_{M^{+}, M, A}$ is an elementary extension of $\mathfrak{B}_{M, M, A}$.

3) $T_{M, A}$ has amalgamation and JEP.

4) $\operatorname{Th}\left(\mathfrak{B}_{M, M, A}\right)$ is the model completion of $T_{M, A}$ so is equal to $T_{M, A}^{*}$ (which is well defined).

5) $T_{M, A}^{*}$ is a dependent (complete first order) theory.

Proof. 1) By Claim 1.7(2), Definition 1.10(1) and $A$ being full over $M$.

2) E.g. use an ultrapower $\mathfrak{C}^{\kappa} / D$ of $\mathfrak{C}$ with $\kappa \geq|T|+|C|+|A|, D$ a regular filter on $\kappa$ and let $\mathbf{j}$ be the canonical embedding of $\mathfrak{C}$ into $\mathfrak{C}^{\kappa} / D$. So we can find 
$f: C \rightarrow M^{\kappa} / D$ such that $f \cup\left(\mathbf{j}\lceil A)\right.$ is an elementary mapping, i.e., a $\left(\mathfrak{C}, \mathfrak{C}^{\kappa} / D\right)$ elementary embedding, now it should be clear.

3) The JEP is trivial because of the individual constants $c_{a}(a \in M)$. The amalgamation property holds by 1.8 as we can replace $M_{1}$ there by any set which is full over $M$.

4) By parts (1),(2),(3) where we have already proved.

5) As $\mathfrak{B}_{M, M, A}$ is a model of it and reflects.

That is, assume $\psi(x, \bar{y})$ is a formula with the independence property in $T_{M, A}^{*}$, then by part (1) without loss of generality $\psi$ is an atomic relation hence for some formula $\varphi(x, \bar{y}, \bar{z}) \in \mathbb{L}\left(\tau_{T}\right)$ and $\bar{c} \in{ }^{\ell g(\bar{z})} A$, for every $a, \bar{b}$ from $M, \mathfrak{C} \models \varphi[a, \bar{b}, \bar{c}]$ iff $\mathfrak{B}_{M, M, A} \models \psi(a, \bar{b})$.

By the choice of $\psi(x, \bar{y})$ for every $n<\omega$ there are $\bar{a}_{\ell}^{n} \in{ }^{\ell g(\bar{y})}\left(\mathfrak{B}_{M, M, A}\right)={ }^{\ell g(\bar{y})}(M)$ for $\ell<\omega$ and $b_{w}^{n} \in \mathfrak{B}_{M, M, A}$, i.e., $b_{w}^{n} \in M$ for $w \subseteq\{0, \ldots, n-1\}$ such that for every $w \subseteq\{0, \ldots, n-1\}$ and $\ell<n$ we have $\mathfrak{B}_{M, M, A} \models \psi\left[b_{w}^{n}, a_{\ell}^{n}\right]^{\text {if }(\ell \in w)}$, hence $\mathfrak{C} \models \varphi\left[b_{w}^{n}, \bar{a}_{\ell}^{n}, \bar{c}\right]^{\mathrm{if}(\ell \in w)}$. So $\varphi(x ; \bar{y}, \bar{z})$ has the independence property in $T$. $\quad \square_{1.12}$

1.13 Conclusion. Assume $M \prec \mathfrak{C}$ and $A \subseteq \mathfrak{C}$. Then $\operatorname{Th}\left(\mathfrak{B}_{M, M, A}\right)$ is a dependent (complete first order) theory.

Proof. By 1.11(4) and 1.12(5) it is the reduct of a dependent (complete first order) theory. More fully let $A_{1}$ be full over $M$ such that $A \subseteq A_{1}$ and let $\kappa=\left|A_{1}\right|+|T|$. We can find a $\kappa^{+}$-saturated elementary extension $\mathfrak{B}^{\prime}$ of $\mathfrak{B}_{M, M, A_{1}}$ and by $1.10(1)$ without loss of generality it is $\mathfrak{B}_{C, M, A_{1}}$ for some $C$, so $M \subseteq C$ and $\operatorname{tp}(C, M \cup$ $\left.A_{1}\right)$ is finitely satisfiable in $M$. Clearly if $\operatorname{Th}\left(\mathfrak{B}_{M, M A}\right)$ is dependent then so is $\operatorname{Th}\left(\mathfrak{B}_{M, M, A_{1}}\right)=\operatorname{Th}\left(\mathfrak{B}^{\prime}\right)$. By $1.12(1),(5)$ we are done.

1.14 Definition. 1) For any model $\mathfrak{B}$ (not necessarily of $T$ ) and $A \subseteq \mathfrak{B}$ let $\mathbb{B}^{m}[A, \mathfrak{B}]$ be the family of subsets of ${ }^{m} A$ of the form $\left\{\bar{a} \in{ }^{m} A: \varphi(\bar{x}, \bar{a}) \in p\right\}$ for some $p \in \mathbf{S}^{m}(A, \mathfrak{B})$.

2) If $\mathfrak{B} \prec \mathfrak{C}$ we may omit $\mathfrak{B}$.

Remark. If $\mathfrak{B}=\mathfrak{C}$ (or just if $\mathfrak{B}$ is $|A|^{+}$-saturated) then $\mathbb{B}^{m}[A, \mathfrak{B}]=\{\{\bar{a}: \mathfrak{B} \models$ $\varphi[\bar{b}, \bar{a}]\}: \varphi(\bar{x}, \bar{y}) \in \mathbb{L}\left(\tau_{\mathfrak{B}}\right)$ and $\left.\bar{b} \in{ }^{\ell g}(\bar{y}) \mathfrak{B}\right\}$.

1.15 Question: Assume $M \subseteq A \subseteq \mathfrak{C}$ and $\mathfrak{B}$ a standard model of $T_{M, A}$ and $N=$ $\mathfrak{B}\left\lceil\tau_{T}\right.$. Then do we have

$(*)_{T, T_{M, A}} \quad$ for any ultrafilter $D_{0}$ on $\mathbb{B}[N, N]$, the number of ultrafilters $D_{1}$ 
on $\mathbb{B}[N, \mathfrak{B}]$ extending it is at most $2^{|T|+|A|}$ ?

1.16 Remark. 1) For complete (first order theories) $T \subseteq T_{1}$, the condition $(*)_{T, T_{1}}$ of 1.15 has affinity to conditions like "any model of $T$ has $<1$ or $\leq \aleph_{0}$ or $<\|M\|$ expansions to a model of $T_{1}$ ". What is the syntactical characterization?

2) When is $\mathfrak{B}_{N, M, A}$ a model of $T_{M, A}^{*}$ ? Assume $T^{*}$ has elimination of quantifiers does the condition implies it, i.e., implies $\mathfrak{B}_{N, M, A} \models T_{M, A}^{*}$ ?

$\square_{N, M, A}$ every formula over $N \cup A$ which does not fork over $N$ is realized in $N$.

1.17 Discussion: 1) Note that in the proof 1.2 we use " $T$ is dependent" just to deduce that the formula $\varphi(x, y, \bar{z})$ is dependent, i.e., for some $n=n_{\varphi(x, y, \bar{z})}$

$$
\circledast \mathfrak{C} \models \neg\left(\exists x_{0} y_{0}, \ldots, x_{n-1} y_{n-1}\right) \bigwedge_{w \subseteq n}(\exists \bar{z}) \bigwedge_{\ell<n} \varphi\left(x_{\ell}, y_{\ell}, \bar{z}\right)^{\mathrm{if}(\ell \in w)} .
$$

We can in the proof use finite $\Delta_{1}, \Delta_{2}$ large enough for $\varphi(x, y, \bar{c})$, i.e., such that for a suitable $n$ :

$\circledast_{2} \Delta_{1}=\left\{(\exists \bar{z})\left(\bigwedge_{\ell<n} \varphi\left(x_{0}, y_{0}, \ldots, x_{n-1}, y_{n-1}, \bar{z}\right)^{\mathrm{if}(\ell \in w)}: w \subseteq n\right\}\right.$.

In particular we need

$\circledast_{3}$ there is $\Delta_{1}$-indiscernible sequence $\left\langle\left(a_{\ell}, b_{\ell}\right): \ell<2 n\right\rangle$ and $\bar{c}^{\prime}$ such that $\mathfrak{C} \models$ $\varphi\left[a_{\ell}, b_{\ell}, \bar{c}^{\prime}\right]$ iff $\ell$ is odd

$\circledast_{4} \Delta_{2}=\left\{\left(\exists y_{0}, y_{2}, \ldots, y_{2 n-2}\right)\left(\wedge q\left(x_{0}, y_{0}, \ldots, x_{2 n-1}, y_{2 n-1}\right): q\right.\right.$ is a complete $\Delta_{1}$-type of a $\Delta_{1}$-indiscernible sequence of pairs of length $\left.2 n\right\}$ hence

$\circledast_{5}$ there is no $\Delta_{2}$ indiscernible sequence $\left\langle\left(a_{2 \ell}, a_{2 \ell+1}, b_{2 \ell+1}\right): \ell<n\right\rangle^{\wedge}\left\langle\left(a_{\omega+2 \ell}, a_{\omega+2 \ell+1}, b_{\omega+2 \ell+1}\right)\right.$ : $\ell<n\rangle$ such that $\mathfrak{C} \models \varphi\left[a_{2 \ell+1}, b_{2 \ell+1}, \bar{c}\right]$ for $\ell<n$ and $\left\{\bar{a}_{2 \ell}, a_{2 \ell}, b_{2 \ell+1}: \ell<\right.$ $n\} \subseteq M$ and for each $\ell<n$ for no $b^{\prime} \in M$ do we have $\models \varphi\left[a_{2 \ell}, b^{\prime}, \bar{c}\right]$.

2) So looking at the proof and 1.7(2)

$\circledast_{6}$ there is a finite set $\Delta=\Delta_{\varphi}^{*}$ of formulas of the form $\psi(x, \bar{z})$ computable from $\varphi(x, y, \bar{z})$ (and $\left.n_{\varphi}\right)$ such that:

(a) if $M, \bar{c}, D$ are as in 1.2 then for some $\bar{c}^{\prime}$ the set $\psi\left(M, \bar{c}^{\prime}\right)$ belongs to $D$ and is included in $\{a \in M$ : for no $b \in M$ do we have $\models \varphi[a, b, \bar{c}]\}$

(b) $\{a \in M:(\exists b \in M)(\varphi(a, b, \bar{c})\}$ is a finite union of sets from $\{\psi(M, \bar{C})$ : $\bar{c}^{\prime} \in \bar{z}^{\prime} \mathfrak{C}$ and $\left.\psi\left(x, \bar{z}^{\prime}\right) \in \Delta\right\}$. 
If in $\circledast_{6}(b)$ there is a bound $n$ on the size of the set not depending on $(M, \bar{c})$ let $\Delta_{\varphi}^{*}=\left\{\psi_{\ell}\left(\bar{x}, \bar{z}_{\ell}\right): \ell<n_{*}\right\}$ and let $\psi^{*}(\bar{x}, \bar{z})=\bigwedge_{\ell>n} z^{n}=z^{\ell} \rightarrow \psi_{\ell}\left(x, \bar{z}_{\ell}\right)$ can serve instead so in $\circledast_{6}$ without loss of generality $\Delta_{\varphi}^{*}=\left\{\psi_{\varphi}^{*}\left(\bar{x}, \bar{z}^{*}\right)\right\}$.

3) We elaborate; we know that if $\mathbf{I}=\left\{a \in{ }^{m} M\right.$ : there is $b \in M$ such that $\mathfrak{C} \models$ $\varphi[a, b, \bar{c}]\}$ where $\varphi=\varphi(x, y, \bar{z}) \in \mathbb{L}\left(\tau_{T}\right), \bar{c} \in \mathfrak{C}, M \prec \mathfrak{C}$ then for some $\psi\left(x, \bar{z}^{\prime}\right) \in$ $\mathbb{L}\left(\tau_{T}\right)$ and $\bar{c}^{\prime} \in \ell g\left(\bar{z}^{\prime}\right) \mathfrak{C}$ we have $\mathbf{I}=\psi\left(M, \bar{c}^{\prime}\right)$. Can we characterize $\psi$ ? Yes, but not so well. Toward this first let $n(*)$ be minimal such that there are no $a_{\ell}, b_{\ell},(\ell<$ $n(*), \bar{c}_{\eta},\left(\eta \in{ }^{(*)} 2\right)$ from $\mathfrak{C}$ such that $M \models \varphi\left(a_{\ell}, b_{\ell}, \bar{c}_{\eta}\right)$ iff $\eta(\ell)=1$.

Let $\psi_{n}\left(x_{0}, y_{0}, \ldots, x_{n(*)-1} \hat{y_{n(*)-1}}\right)=(\exists \bar{z}) \bigwedge_{\ell<n(*)} \varphi\left(x_{\ell}, y_{\ell}, \bar{z}\right)^{\eta(\ell)}$ for $\eta \in{ }^{n(*)} 2$ and $\Delta_{1}=\left\{\psi_{\eta}\left(\bar{x}_{0}, \bar{y}_{0}, \ldots, \bar{x}_{n(*)}, \bar{y}_{n(*)-1}\right)\right\}$. Let $\Delta_{2}$ be the closure of $\Delta_{1}$ under permuting the variables.

Let $\Delta_{3, k}$ be the set of formulas of the form $\vartheta\left(y_{2 k(*)} ; x_{0}, y_{0}, x_{1}, y_{1}, \ldots, x_{2 k-1}, y_{2 k-1}-\right.$ $\left.y_{2 k} ; x_{2 k+1}, y_{2 k+1}, \ldots, x_{2 n(*)-2}, x_{2 n(*)-1}, y_{2 n(*)-1}\right)=\left(\exists y_{2 k+2}\right) \ldots\left(\exists y_{2 n(*)_{2}}\right) \psi^{*}$ where $\psi^{*}$ is a conjunction or formula from $\Delta_{2}$ and their negation.

Now $\psi$ belongs to $\Delta_{3, k}$ for some $k<n(*)$. (In fact we could be somewhat more specific).

Why? We work with $\cup \Delta_{3, \ell}$ choose $a_{2 \ell}, a_{2 \ell+1}, k_{2 \ell+1}$ as in the proof for it. Then we choose $b_{2 \ell+1} \in M$ by induction on $\ell<n(*)$ such that $\left\langle\left(a_{\ell}, b_{\ell}\right): \ell<2 n(*)\right\rangle$ is $\Delta_{1}$-indiscernible. So for every $\eta \in{ }^{(*)} 2$ we have $(\exists \bar{z}) \bigwedge \varphi\left(a_{\ell}, b_{\ell}, \bar{z}\right)^{\eta(\ell)}$. 


\section{$\S 2$ MORE ON INDISCERNIBLE SEQUENCES}

2.1 Context. 1) $T$ is a (first order complete) dependent theory.

2) $\mathfrak{C}$ is the monster model of $T$.

This section is complimentary to $[\mathrm{Sh} 715, \S 5]$ so recall the definition.

2.2 Definition. Let $\overline{\mathbf{a}}^{\ell}=\left\langle\bar{a}_{t}^{\ell}: t \in I_{\ell}\right\rangle$ be an indiscernible sequence which is endless (i.e., $I_{\ell}$ having no last element) for $\ell=1,2$.

1) We say that $\overline{\mathbf{a}}^{1}, \overline{\mathbf{a}}^{2}$ are perpendicular when:

(*) if $\bar{b}_{n}^{\ell}$ realizes $\operatorname{Av}\left(\left\{\bar{b}_{m}^{k}\right.\right.$ : we have $m<n \quad \& \quad k \in\{1,2\}$ or we have $m=n$ \& $k<\ell\} \cup \overline{\mathbf{a}}^{1} \cup \overline{\mathbf{a}}^{2}, \overline{\mathbf{a}}^{\ell}$ ) for $\ell=1,2$ then $\overline{\mathbf{b}}^{1}, \overline{\mathbf{b}}^{2}$ are mutually indiscernible (i.e., each is indiscernible over the set of elements appearing in the other) where $\overline{\mathbf{b}}^{\ell}=\left\langle\bar{b}_{n}^{\ell}: n<\omega\right\rangle$ for $\ell=1,2$.

We define " $\Delta$-perpendicular" in the obvious way.

2) We say $\overline{\mathbf{a}}^{1}, \overline{\mathbf{a}}^{2}$ are equivalent and write $\approx$ if for every $A \subseteq \mathfrak{C}$ we have $\operatorname{Av}\left(A, \overline{\mathbf{a}}^{1}\right)=$ $\operatorname{Av}\left(A, \overline{\mathbf{a}}^{2}\right)$.

3) If $\overline{\mathbf{a}}^{1} \subseteq A$ we let dual-cf( $\left(\overline{\mathbf{a}}^{1}, A\right)=\operatorname{Min}\left\{|B|: B \subseteq A\right.$ and no $\bar{c} \in{ }^{\omega>} A$ realizes $\left.\operatorname{Av}\left(B, \overline{\mathbf{a}}^{1}\right)\right\}$; we usually apply this when $A=M$.

2.3 Claim. Assume

$(\alpha) \overline{\mathbf{b}}=\left\langle\bar{b}_{t}: t \in I_{0}\right\rangle$ is an infinite indiscernible sequence over $A$

( $\beta) B \subseteq \mathfrak{C}$.

$\underline{\text { Then }}$ we can find $I_{1}, J$ and $\bar{b}_{t}$ for $t \in I_{1} \backslash I_{0}$ such that:

(a) $I_{0} \subseteq I_{1}, I_{1} \backslash I_{0} \subseteq J \subseteq I_{1}$ and $\left|I_{1} \backslash I_{0}\right| \leq|J| \leq|B|+|T|$

(b) $\overline{\mathbf{b}}^{\prime}=\left\langle\bar{b}_{t}: t \in I_{1}\right\rangle$ is an indiscernible sequence over $A$

(c) if $I_{2}$ is a $J$-free extension of $I_{1}$ (see below) and $\bar{b}_{t}$ for $t \in I_{2} \backslash I_{1}$ are such that $\overline{\mathbf{b}}^{\prime \prime}=\left\langle\bar{b}_{t}: t \in I_{2}\right\rangle$ is an indiscernible sequence over $A \underline{\text { then }}$

$\circledast \quad$ if $n<\omega, \bar{s}, \bar{t} \in{ }^{n}\left(I_{2}\right)$ and $\bar{s} \sim_{J} \bar{t}$ (see below), then $\bar{b}_{\bar{s}}, \bar{b}_{\bar{t}}$ realize the same type over $A \cup B$ where $\bar{b}_{\left\langle t_{\ell}: \ell<n\right\rangle}=\bar{b}_{t_{0}}{ }^{\wedge} \bar{b}_{t_{1}}{ }^{\wedge}{ }^{\cdots}{ }^{\wedge} \bar{b}_{t_{n-1}}$. 
2.4 Definition. 1) For linear orders $J, I_{1}, I_{2}$ we say that $I_{2}$ is a $J$-free extension of $I_{1}$ when: $J \subseteq I_{1} \subseteq I_{2}$ and

$\circledast$ if $t \in I_{2} \backslash I_{1}$ and $s \in J$ then for some $t^{\prime} \in I_{1}$ we have $I_{2} \models s<t^{\prime}<t$ or $I_{2} \models t<t^{\prime}<s$.

2) For linear orders $J, I_{1}, I_{2}$ we say that $I_{2}$ is a strong $J$-free extension of $I_{1}$ when $J \subseteq I_{1} \subseteq I_{2}$ and:

$\circledast$ if $t \in I_{2} \backslash I_{1}$ then for some $s_{1}, s_{2} \in I_{1}$ we have $s_{1}<_{I_{2}} t<_{I_{2}} s_{2}$ and $\left[s_{1}, s_{2}\right]_{I_{1}} \cap$ $J=\emptyset$.

3) For linear orders $J \subseteq I$ and $\bar{s}, t \in{ }^{n} I$ let $\bar{s} \sim_{J} \bar{t}$ mean that $\left(s_{\ell}<_{I} s_{k}\right) \equiv\left(t_{\ell}<_{I} t_{k}\right)$ and $\left(s_{\ell}<_{I} r\right) \equiv\left(t_{\ell}<_{I} r\right)$ and $\left(r<_{I} s_{\ell} \equiv r<_{I} t_{\ell}\right)$ whenever $\ell, k<n$ and $\left.r \in J\right)$. Similarly for $\bar{s}, \bar{t} \in{ }^{\alpha} I$.

2.5 Remark. In 2.3 why do we need " $J$-free"? Let $M=\left(\mathbb{R},<, Q^{M}\right), Q^{M}=\mathbb{Q}, B=$ $\{0\}, A=\emptyset, I_{0}$ the irrationals, $b_{t}=t$ for $t \in I_{0}$.

Proof.

Proof of 2.3 .

We try to choose by induction on $\zeta<\lambda^{+}$where $\lambda=|T|+|B|$ a sequence $\overline{\mathbf{b}}^{\zeta}=\left\langle\bar{b}_{t}: t \in J_{\zeta}\right\rangle$ and together with $\overline{\mathbf{b}}^{\zeta+1}$ we choose $n_{\zeta}, \bar{s}_{\zeta}, \bar{t}_{\zeta}, J_{\zeta}^{\prime}, \varphi_{\zeta}, \bar{c}_{\zeta}, \bar{d}_{\zeta}$ such that

(a) $J_{\zeta}$ is a linear order, increasing continuous with $\zeta$

(b) $J_{0}=I_{0}$ (so $\overline{\mathbf{b}}^{0}=\overline{\mathbf{b}}$ ), $J_{\varepsilon+1} \backslash J_{\varepsilon}$ is finite so $\left|J_{\varepsilon} \backslash I_{0}\right|<|\varepsilon|^{+}+\aleph_{0}$

(c) $\overline{\mathbf{b}}^{\zeta}$ is an indiscernible sequence over $A$

(d) $J_{\zeta}^{\prime} \subseteq J_{\zeta}, J_{\zeta}=I_{0} \cup J_{\zeta}^{\prime}, J_{\zeta}^{\prime}$ is increasing continuous with $\zeta$ and $\left|J_{\zeta}^{\prime}\right|<|\zeta|^{+}+\aleph_{0}$

(e) if $\zeta=\varepsilon+1 \underline{\text { then }} n_{\varepsilon}<\omega, \bar{s}_{\varepsilon} \in n_{\varepsilon}\left(J_{\zeta}^{\prime}\right), \bar{t}_{\varepsilon} \in n_{\varepsilon}\left(J_{\zeta}^{\prime}\right), \varphi_{\varepsilon}=\varphi_{\varepsilon}\left(\bar{x}_{0}, \ldots, \bar{x}_{n_{\varepsilon}-1}, \bar{c}_{\varepsilon}, \bar{d}_{\varepsilon}\right), \bar{c}_{\varepsilon} \subseteq$ $B, \bar{d}_{\varepsilon} \subseteq A$ and $J_{\zeta}^{\prime}=J_{\varepsilon}^{\prime} \cup\left(\bar{s}_{\varepsilon}{ }^{\wedge} \bar{t}_{\varepsilon}\right)$

(f) $\bar{s}_{\varepsilon} \sim J_{\varepsilon}^{\prime} \bar{t}_{\varepsilon}$ and $\models \varphi_{\varepsilon}\left[\bar{b}_{\bar{s}_{\varepsilon}}, \bar{c}_{\varepsilon}, \bar{d}_{\varepsilon}\right] \wedge \neg \varphi_{\varepsilon}\left[\bar{b}_{\bar{t}_{\varepsilon}}, \bar{c}_{\varepsilon}, \bar{d}_{\varepsilon}\right]$

(g) $J_{\zeta+1}$ is a $J_{\zeta}^{\prime}$-free extension of $J_{\zeta}$.

If we succeed, for some unbounded $w \subseteq \lambda^{+}$and $n_{*}, \varphi_{\xi}, \bar{c}^{*}$ and $u$ for every $\varepsilon \in w$ we have $n_{\varepsilon}=n_{*}, \varphi_{\varepsilon}=\varphi_{*}, \bar{c}_{\varepsilon}=\bar{c}^{*}$ and $u=\left\{\ell<n_{*}: s_{\varepsilon, \ell} \in J_{\zeta}^{\prime}\right\}$. Now let $J^{*}=\cup\left\{J_{\zeta}^{\prime}: \zeta<\lambda^{+}\right\}$, so every $J^{\prime} \subseteq J^{*}$ of cardinality $\leq \lambda$ is included in $J_{\zeta}^{\prime}$ for some $\zeta<\lambda^{+}$and we get contradiction to clause (b) of [Sh 715, 3.2] $(=3.4 \mathrm{t})$, hence we fail, i.e., we cannot choose for some $\zeta$. But we can choose $\overline{\mathbf{b}}_{\zeta}=\left\langle b_{t}: t \in J_{\zeta}\right\rangle$, if 
$\zeta=0$ by clause (b) and if $\zeta$ is a limit ordinal by clause (a). So $\zeta=\varepsilon+1$, we have chosen $\overline{\mathbf{b}}=\left\langle\bar{b}_{t}: t \in J_{\zeta}\right\rangle$ but we cannot choose $J_{\zeta+1}, \overline{\mathbf{b}}^{\zeta+1}, n_{\zeta}, \bar{s}_{\zeta}, \bar{t}_{\zeta}, J_{\zeta}^{\prime}, \varphi_{\zeta}, \bar{c}_{\zeta}, \bar{d}_{\zeta}$ as required. Then $\overline{\mathbf{b}}^{\varepsilon}$ is as required.

The aim of $2.6+2.9$ below is to show a complement of [Sh 715, §5]; that is, in the case of small cofinality what occurs in one cut is the "same" as what occurs in others.

2.6 Claim. Assume

(a) $\mu \geq|T|$

(b) $I_{\ell}$ for $\ell<4$ are pairwise disjoint linear orders

(c) $I_{\ell}=\bigcup_{\beta<\mu^{+}} I_{\ell}^{\beta}, I_{\ell}^{\beta}$ (strictly) increasing with $\beta$ and $\left|I_{\ell}^{\beta}\right| \leq \mu$ for $\ell<4$

(d) $(\alpha) \quad \ell \in\{0,2\} \Rightarrow I_{\ell}^{\beta}$ an end segment of $I_{\ell}$

( $\beta) \quad \ell \in\{1,3\} \Rightarrow I_{\ell}^{\beta}$ is an initial segment of $I_{\ell}$

(e) $I=I_{0}+I_{1}+I_{2}+I_{3}$ and $I^{\beta}=I_{0}^{\beta}+I_{1}^{\beta}+I_{2}^{\beta}+I_{3}^{\beta}$

$(f)\left\langle\bar{b}_{t}: t \in I\right\rangle$ is an indiscernible sequence.

$\underline{\text { Then }}$ we can find a limit ordinal $\beta(*)<\mu^{+}$and $\left\langle\bar{b}_{t}^{*}: t \in I\right\rangle$ such that:

(A) $\bar{b}_{t}^{*}=\bar{b}_{t}$ if $t \in I \backslash I^{\beta(*)}$

$(B)_{1}\left\langle\bar{b}_{t}^{*}: t \in I \backslash I_{0}^{\beta(*)} \backslash I_{1}^{\beta(*)}\right\rangle$ is an indiscernible sequence

$(B)_{2}\left\langle\bar{b}_{t}^{*}: t \in I \backslash I_{2}^{\beta(*)} \backslash I_{3}^{\beta(*)}\right\rangle$ is an indiscernible sequence

$(C)_{1} \operatorname{tp}_{*}\left(\left\langle\bar{b}_{t}^{*}: t \in I_{0}^{\beta(*)} \cup I_{1}^{\beta(*)}\right\rangle, \cup\left\{\bar{b}_{t}^{*}: t \in\left(I \backslash I^{\beta}\right) \cup I_{2}^{\beta(*)} \cup I_{3}^{\beta(*)} \cup\left(I_{0}^{\beta(*)+\omega} \backslash I_{0}^{\beta(*)}\right) \cup\right.\right.$ $\left.\left.\left(I_{1}^{\beta(*)+\omega} \backslash I_{1}^{\beta(*)}\right)\right\}\right) \vdash$

$\operatorname{tp}_{*}\left(\left\langle\bar{b}_{t}^{*}: t \in I_{0}^{\beta(*)} \cup I_{1}^{\beta(*)}\right\rangle, \cup\left\{\bar{b}_{t}^{*}: t \in\left(I \backslash I^{\beta(*)}\right) \cup I_{2}^{\beta(*)} \cup I_{3}^{\beta(*)}\right\}\right)$

for any $\beta \in\left[\beta(*)+\omega, \mu^{+}\right)$

$(C)_{2} \operatorname{tp}_{*}\left(\left\langle\bar{b}_{t}^{*}: t \in I_{2}^{\beta(*)} \cup I_{3}^{\beta(*)}\right\rangle, \cup\left\{\bar{b}_{t}^{*}: t \in\left(I \backslash I^{\beta}\right) \cup I_{0}^{\beta(*)} \cup I_{1}^{\beta(*)} \cup\left(I_{2}^{\beta(*)+\omega} \backslash I_{2}^{\beta(*)}\right) \cup\right.\right.$ $\left.\left.\left(I_{3}^{\beta(*)+\omega} \backslash I_{3}^{\beta(*)}\right)\right\}\right) \vdash$

$\operatorname{tp}\left(\left\langle b_{t}^{*}: t \in I_{2}^{\beta(*)} \cup I_{3}^{\beta(*)}\right\rangle, \cup\left\{\bar{b}_{t}^{*}: t \in\left(I \backslash I^{\beta(*)}\right) \cup I_{0}^{\beta(*)} \cup I_{1}^{\beta(*)}\right\}\right)$

for any $\beta \in\left[\beta(*)+\omega, \mu^{+}\right)$

$(D)_{1}\left\langle\bar{b}_{t}^{*}: t \in I_{0} \backslash I_{0}^{\beta(*)}\right\rangle$ is an indiscernible sequence over $\cup\left\{\bar{b}_{t}^{*}: t \in I_{0}^{\beta(*)} \cup I_{1} \cup\right.$ $\left.I_{2} \cup I_{3}\right\}$

$(D)_{2}\left\langle\bar{b}_{t}^{*}: t \in\left(I_{1} \backslash I_{1}^{\beta(*)}\right)+\left(I_{2} \backslash I_{2}^{\beta(*)}\right)\right\rangle$ is an indiscernible sequence over $\cup\left\{\bar{b}_{t}^{*}: t \in\right.$ $\left.I_{0} \cup I_{1}^{\beta(*)} \cup I_{2}^{\beta(*)} \cup I_{3}\right\}$ 
$(D)_{3}\left\langle\bar{b}_{t}^{*}: t \in I_{3} \backslash I_{3}^{\beta(*)}\right\rangle$ is an indiscernible sequence over $\cup\left\{\bar{b}_{t}^{*}: t \in I_{0} \cup I_{1} \cup I_{2} \cup\right.$ $\left.I_{3}^{\beta(*)}\right\}$.

2.7 Remark. What occurs if $T$ is stable (or just $\overline{\mathbf{b}}$ is)? We get something like $\left\{\bar{b}_{t}^{*}: t \in I_{0}^{\beta(*)} \cup I_{1}^{\beta(*)}\right\}=\left\{\bar{b}_{t}^{*}: t \in I_{2}^{\beta(*)} \cup I_{3}^{\beta(*)}\right\}$.

Proof. For simplicity assume $I_{\ell}^{0}=\emptyset$.

We choose by induction on $n<\omega$ an ordinal $\beta(n)$ and $\left\langle\bar{b}_{t}^{n}: t \in I\right\rangle$ such that:

( $\alpha) \beta(n)<\mu^{+}, \beta(0)=0, \beta(n)+\omega \leq \beta(n+1)$

(B) $\bar{b}_{t}^{n}=\bar{b}_{t}$ if $t \in I \backslash I^{\beta(n)}$ or if $n=0$

$(\gamma)_{1}\left\langle\bar{b}_{t}^{n}: t \in I \backslash I_{0}^{\beta(n)} \backslash I_{1}^{\beta(n)}\right\rangle$ realizes the same type as $\left\langle\bar{b}_{t}: t \in I \backslash I_{0}^{\beta(n)} \backslash I_{1}^{\beta(n)}\right\rangle$

$(\gamma)_{2}\left\langle\bar{b}_{t}^{n}: t \in I \backslash I_{2}^{\beta(n)} \backslash I_{3}^{\beta(n)}\right\rangle$ realizes the same type as $\left\langle\bar{b}_{t}: t \in I \backslash I_{2}^{\beta(n)} \backslash I_{3}^{\beta(n)}\right\rangle$

$(\delta)_{1}$ if $n$ is even then:

(1) $\bar{b}_{t}^{n+1}=\bar{b}_{t}^{n}$ for $t \in I \backslash I_{2}^{\beta(n)} \backslash I_{3}^{\beta(n)}$

(2) if $\beta(n+1)<\beta<\mu^{+}$then the type which $\left\langle\bar{b}_{t}^{n+1}: t \in I_{2}^{\beta(n)} \cup I_{3}^{\beta(n)}\right\rangle$ realizes over $\cup\left\{\bar{b}_{t}^{n}: t \in\left(I_{0} \backslash I_{0}^{\beta}\right) \cup I_{0}^{\beta(n+1)} \cup\left(I_{1} \backslash I_{1}^{\beta}\right)\right.$ $\left.\cup I_{1}^{\beta(n+1)} \cup\left(I_{2} \backslash I_{2}^{\beta}\right) \cup\left(I_{2}^{\beta(n+1)} \backslash I_{2}^{\beta(n)}\right) \cup\left(I_{3} \backslash I_{3}^{\beta}\right) \cup\left(I_{3}^{\beta(n+1)} \backslash I_{3}^{\beta(n)}\right)\right\}$ has a unique extension over $\cup\left\{\bar{b}_{t}^{n}: t \in I \backslash I_{2}^{\beta(n)} \backslash I_{3}^{\beta(n)}\right\}$

(3) $\bar{b}_{t}^{n+1}=b_{t}^{n}$ if $t \in I_{2}^{\beta(k)} \cup I_{3}^{\beta(k)}, k<n$

$(\delta)_{2}$ if $n$ is odd like $\left(\delta_{1}\right)$ inverting the roles of $\left(I_{0}, I_{1}\right),\left(I_{2}, I_{3}\right)$

$(\varepsilon)\left\langle\bar{b}_{t}^{n}: t \in I\right\rangle$ satisfies clauses $(D)_{1},(D)_{2},(D)_{3}$ of the claim with $\beta(n)$ instead of $\beta(*)$.

The induction step is as in the proof of 2.3 (though we use the finite character for the middle clause $(2)$ of clauses $\left.(\delta)_{1},(\delta)_{2}\right)$.

Alternatively, letting $n$ be even we try to choose $\beta_{n}(\varepsilon), \overline{\mathbf{b}}^{n, \varepsilon}=\left\langle\bar{b}_{t}^{n, \varepsilon}: t \in I_{2}^{\beta(n)}+\right.$ $\left.I_{3}^{\beta(n)}\right\rangle$ by induction on $\varepsilon \leq \mu^{+}$such that:

$\bigodot(a) \beta_{n}(\varepsilon)<\mu^{+}$

(b) $\beta_{n}(0)=\beta(n)$

(c) $\beta_{n}(\varepsilon)$ is increasing and continuous

(d) $\left.\zeta<\varepsilon \Rightarrow \operatorname{tp}\left(\overline{\mathbf{b}}^{n, \varepsilon}\right), \cup\left\{b_{t}^{n}: t \in\left(I \backslash I_{\beta_{n}(\varepsilon)}^{\varepsilon}\right) \cup I_{\beta_{n}(\zeta)}\right\}\right) \vdash$ $\operatorname{tp}\left(\overline{\mathbf{b}}^{n, \zeta}, \cup\left\{\bar{b}_{t}^{n}: t \in\left(I \backslash I_{\beta_{n}(\varepsilon)}\right) \cup I_{\beta_{n}(\zeta)}\right\}\right.$ 
(e) if $\varepsilon=\zeta+1$, then $(\delta)_{1}(2)$ fails if we let

$$
\bar{b}_{t}^{n+1}=\left\{\begin{array}{ll}
b_{t}^{n} & \text { if } t \in I \backslash I_{2}^{\beta(n)} \backslash I_{3}^{\beta(n)} \\
\bar{b}_{t}^{n, \zeta} & \text { if } t \in I_{2}^{\beta(n)} \cup I_{3}^{\beta(n)}
\end{array} .\right.
$$

If we succeed to carry the induction, by [Sh 715], for some $\varepsilon$ the sequences $\left\langle\bar{b}_{t}^{n}: t \in\right.$ $\left.I_{0}^{\beta_{n}(\varepsilon)}\right\rangle,\left\langle\bar{b}_{t}^{n}: t \in I_{1}^{\beta_{n}(\varepsilon)}+I_{2}^{\beta_{n}(\varepsilon)}\right\rangle,\left\langle\bar{b}_{t}^{n}: t \in I_{3}^{\beta_{n}(\varepsilon)}\right\rangle$ are mutually indiscernible over $\cup\left\{\bar{b}_{t}^{n, \mu^{+}}: t \in I_{2}^{\beta(n)}+I_{3}^{\beta(n)}\right\} \cup\left\{b_{t}^{n}: t \in\left(I \backslash I_{\beta_{n}(\varepsilon)}\right)\right\}$ (because $\left\langle\bar{b}_{t}: t \in I_{0} \backslash I_{0}^{\beta_{n}(\varepsilon)}\right\rangle,\left\langle\bar{b}_{t}\right.$ : $\left.t \in\left(I_{1} \backslash I_{\beta_{n}(\varepsilon)}\right)+I_{2} \backslash I_{2}^{\beta_{n}(\varepsilon)}\right\rangle,\left\langle\bar{b}_{t}: t \in I_{3} \backslash I_{3}^{\beta_{n}(\varepsilon)}\right\rangle$ are mutually indiscernible, recalling $(\beta)$.

This contradicts $(e)$. So we cannot complete the induction. We certainly succeed for $\varepsilon=0$, and there is no problem for limit $\varepsilon \leq \mu^{+}$. So for some $\varepsilon=\zeta+1$ we have succeed for $\zeta$ and cannot choose for $\varepsilon$. We define $\bar{b}_{i}^{n+1}$ as in $(e)$ of $\odot$ above, and choose $\beta(n+1) \in\left[\beta_{n}(\varepsilon), \mu^{+}\right)$such that clauses $(\varepsilon)$ holds.

Let $\beta(*)=\cup\{\beta(n): n<\omega\}<\mu^{+}, \bar{b}_{t}^{*}$ is $\bar{b}_{t}^{n}$ for every $n$ large enough (exists by clause $(\beta)$ if $t \in I \backslash I^{\beta(*)}$ and by $(\delta)_{\ell}(1)+(3)$ if $\left.t \in I^{\beta(*)}\right)$.

Clearly we are done.

2.8 Claim. Assume

(a) $I, I^{\beta}, I_{\ell}, I_{\ell}^{\beta}$ for $\ell<4, \beta<\mu^{+}$are as in the assumption of claim 2.6

(b) $\beta(*)$ and $\left\langle\bar{b}_{t}^{*}: t \in I\right\rangle$ are as in the conclusion of claim 2.6

(c) $J^{+}=J_{0}^{+}+J_{1}^{+}+J_{2}^{+}+J_{3}^{+}+J_{4}^{+}$linear orders

(d) $J=J_{0}+J_{1}+J_{2}+J_{3}+J_{4}$ linear orders

(e) $J_{1}=J_{1}^{+}+I_{0}^{\beta(*)}+I_{1}^{\beta(*)}$ and $J_{3}=I_{2}^{\beta(*)}+I_{3}^{\beta(*)}$

(f) $J_{0} \subseteq J_{0}^{+}$and $I_{0} \backslash I_{0}^{\beta(*)} \subseteq J_{0}^{+}$

(g) $J_{2} \subseteq J_{2}^{+}$and $\left(I_{1} \backslash I_{1}^{\beta(*)}\right)+\left(I_{2} \backslash I_{2}^{\beta(*)}\right) \subseteq J_{2}$

(h) $J_{4} \subseteq J_{4}^{+}$and $\left(I_{3} \backslash I_{3}^{\beta(*)}\right) \subseteq J_{4}^{+}$

(i) $\left\langle\bar{b}_{t}^{*}: t \in J^{+}\right\rangle$is an indiscernible sequence.

1) If $J_{0}^{\prime}, J_{2}^{\prime}, J_{4}^{\prime}$ are infinite initial segments of $J_{0}, J_{2}, J_{4}$ respectively then

$(\alpha) \operatorname{tp}\left(\left\langle\bar{b}_{t}^{*}: t \in J_{3}\right\rangle, \cup\left\{\bar{b}_{s}: s \in J_{0}^{\prime} \cup J_{1} \cup J_{2}^{\prime} \cup J_{4}^{\prime}\right) \vdash \operatorname{tp}\left(\left\langle\bar{b}_{t}^{*}: t \in J_{3}\right\rangle, \cup\left\{\bar{b}_{s}^{*}: s \in\right.\right.\right.$ $\left.J_{0} \cup J_{1} \cup J_{2} \cup J_{4}\right)$

( $\beta)$ like $(\alpha)$ interchanging $J_{3}, J_{1}$.

2) If $J_{0}$ has no first element, $J_{0}^{\prime} \subseteq J_{0}$ is unbounded from below, $J_{2}^{\prime} \subseteq J_{2}$ is infnite and $J_{4}$ has no last element and $J_{4}^{\prime} \subseteq J_{4}$ is unbounded from above, then the conclusions of (1) holds 
$(\alpha) \operatorname{tp}\left(\left\langle\bar{b}_{t}^{*}: t \in J_{3}\right\rangle, \bigcup\left\{\bar{b}_{s}: s \in J_{0}^{\prime} \cup J_{1} \cup J_{2}^{\prime} \cup J_{4}^{\prime}\right) \vdash \operatorname{tp}\left(\left\langle\bar{b}_{t}^{*}: t \in J_{3}\right\rangle, \cup\left\{\bar{b}_{s}^{*}: s \in\right.\right.\right.$ $\left.\left.J_{0} \cup J_{1} \cup J_{2} \cup J_{4}\right\}\right)$

( $\beta) \operatorname{tp}\left(\left\langle\bar{b}_{t}^{*}: t \in J_{1}\right\rangle, \bigcup\left\{\bar{b}_{s}: s \in J_{0}^{\prime} \cup J_{2}^{\prime} \cup J_{3} \cup J_{4}^{\prime}\right) \vdash \operatorname{tp}\left(\left\langle\bar{b}_{t}^{*}: t \in J_{1}\right\rangle, \cup\left\{\bar{b}_{s}^{*}: s \in\right.\right.\right.$ $\left.\left.J_{0} \cup J_{2} \cup J_{3} \cup J_{4}\right\}\right)$.

3) If $J_{0}^{*}, J_{2}^{*}, J_{4}^{*}$ has neither first element nor last element and $J_{0}^{\prime}, J_{2}^{\prime}, J_{4}^{\prime}$ are subsets of $J_{0}, J_{2}, J_{4}$ respectively unbounded from below and $J_{0}^{\prime \prime}, J_{2}^{\prime \prime}, J_{4}^{\prime \prime}$ are subsets of $J_{0}, J_{2}, J_{4}$ respectively unbounded from above, then the conclusion of part (1) holds.

Proof. The result follows by the local character of $\vdash$ and by the indiscernibility demands in 2.6, i.e., clauses $(D)_{1},(D)_{2},(D)_{3}$.

2.9 Conclusion. 1) If $\mu \geq \kappa \geq|T|$, then for some linear order $J^{*}$ of cardinality $\kappa$ we have

$\bigotimes_{\overline{\mathbf{b}}^{*}, J^{*}}$ we can find $\bar{b}_{t} \in{ }^{m} M$ for $t \in J_{3}$ such that $\left\langle\bar{b}_{t}: t \in J \backslash J_{1}\right\rangle$ is an indiscernible sequence when:

(a) $J=J_{0}+J_{1}+J_{2}+J_{3}+J_{4}$

(b) the cofinalities of $J_{0}, J_{2}, J_{4}$ and their inverse are $\leq \mu$ but are infinite

(c) $J_{1} \cong J^{*}$ and $J_{3} \cong J^{*}$ (hence $J_{1}, J_{3}$ have cardinality $\leq \kappa$ )

(d) $\left\langle\bar{b}_{t}: t \in J \backslash J_{3}\right\rangle$ is an indiscernible sequence (of $m$-tuples)

(e) $M$ is a $\mu^{+}$-saturated model

(f) $\cup\left\{\bar{b}_{t}: t \in J \backslash J_{3}\right\} \subseteq M$.

2) If we allow $J^{*}$ to depend on $\operatorname{tp}^{\prime}\left(\overline{\mathbf{b}}^{*}\right)$, see Definition $0.1(1)$, then we can use $J^{*}$ of the form $\delta^{*}+\delta, \delta<\gamma$

$\left(\delta^{*}\right.$ - the inverse of $\left.\delta\right)$.

Proof. Let $\overline{\mathbf{b}}^{*}$ be an infinite indiscernible sequence.

Let $J_{0}, J_{2}, J_{4}$ be disjoint linear orders as in (b). Apply 2.6 with $I_{1}, I_{3}$ isomorphic to $\left(\mu^{+},<\right)$and $I_{0}, I_{2}$ isomorphic to $\left(\mu^{+},>\right)$, say $I_{\ell}=\left\{t_{\alpha}^{\ell}: \alpha<\mu^{+}\right\}$with $t_{\alpha}^{\ell}$ increasing with $\alpha$ if $\ell \in\{1,3\}$ and decreasing with $\alpha$ if $\ell \in\{0,2\}$, we get $\overline{\mathbf{b}}^{*}=\left\langle b_{t}^{*}\right.$ : $\left.t \in \sum_{\ell<4} I_{\ell}\right\rangle, \beta(*)$ as there with $\operatorname{tp}^{\prime}\left(\overline{\mathbf{b}}^{*} \uparrow I_{0}\right)=\operatorname{tp}^{\prime}\left(\overline{\mathbf{b}}^{\circledast}\right)$, see Definition 0.1. Let $J_{0}^{+}=$ $J_{0}+\left(I_{0} \backslash I_{0}^{\beta(*)}\right), J_{1}^{+}=J_{1}=I_{0}^{\beta(*)}+I_{1}^{\beta(*)}, J_{2}^{+}=J_{2}+\left(I_{1} \backslash I_{1}^{\beta(*)}\right)+\left(I_{2} \backslash I_{2}^{\beta(*)}\right), J_{3}^{+}=$ $I_{2}^{\beta(*)}+I_{3}^{\beta(*)}+J_{3}$ and $J_{4}^{+}=J_{4}+\left(I_{3} \backslash I_{3}^{\beta(*)}\right)$ and $J^{+}=J_{0}^{+}+J_{1}^{+}+J_{2}^{+}+J_{3}^{+}+J_{4}^{+}$. All $J_{\ell}$ are infinite linear orders, choose $J^{*}=J_{1}$, clearly $J_{3} \cong J^{*}$. Now 
$(*)\left\langle\bar{b}_{t}^{*}: t \in J \backslash J_{3}\right\rangle$ is an indiscernible sequence and

$(* *)$ if $M \supseteq \cup\left\{\bar{b}_{t}^{*}: t \in J \backslash J_{3}\right\}$ is $\mu^{+}$-saturated then we can find $\bar{b}_{t}^{\prime} \in{ }^{m} M$ for $t \in J_{3}$ such that

$$
\left\langle\bar{b}_{t}^{*}: t \in J_{0}\right\rangle^{\wedge}\left\langle\bar{b}_{t}^{\prime}: t \in J_{3}\right\rangle^{\wedge}\left\langle\bar{b}_{t}^{*}: t \in J_{4}\right\rangle
$$

is an indiscernible sequence.

[Why? Choose $J_{0}^{\prime} \subseteq J_{0}$ unbounded from below of cardinality $\operatorname{cf}\left(J_{0},>_{J_{0}}\right)$ which is $\leq \mu$ but $\geq \aleph_{0}$ and similarly $J_{2}^{\prime} \subseteq J_{2}, J_{4}^{\prime} \subseteq J_{4}$ and choose $J_{0}^{\prime \prime} \subseteq J_{0}$ unbounded from above of cardinality $\operatorname{cf}\left(J_{0}\right)$ which is $\leq \mu$ and similarly $J_{2}^{\prime \prime} \subseteq J_{2}, J_{4}^{\prime \prime} \subseteq J_{4}$ (all O.K. by clause (b) of the assumption).

Now $p=\operatorname{tp}\left(\left\langle b_{t}^{*}: t \in J_{3}\right\rangle, \cup\left\{\bar{b}_{s}: s \in J_{0}^{\prime} \cup J_{0}^{\prime \prime} \cup J_{2}^{\prime} \cup J_{2}^{\prime \prime} \cup J_{4}^{\prime} \cup J_{4}^{\prime}\right\}\right)$ is a type of cardinality $\leq|T|+\left|J_{0}^{\prime}\right|+\left|J_{0}^{\prime \prime}\right|+\left|J_{2}^{\prime}\right|+\left|J_{2}^{\prime \prime}\right|+\left|J_{4}^{\prime}\right|+\left|J_{4}^{\prime \prime}\right| \leq \mu$ hence is realized by some sequence $\left\langle\bar{b}_{t}^{\prime}: t \in J_{3}\right\rangle$ from $M$.

By Claim 2.8 the desired conclusion in (**) holds.]

So we have gotten the desired conclusion for any $\left\langle J_{\ell}: \ell \leq 4\right\rangle$ and indiscernible sequence, $\overline{\mathbf{b}}=\left\langle\bar{b}_{t}: t \in J \backslash J_{5}\right\rangle$ as long as $\operatorname{tp}^{\prime}(\overline{\mathbf{b}})=\operatorname{tp}^{\prime}\left(\overline{\mathbf{b}}^{*}\right)$ and the order type of $J_{1}, J_{3}$ is as required for $\left.\overline{\mathbf{b}}^{*}\right)$. This is enough for part (2), we are left with (1).

Note that by the proof of 2.3 , the set of $\beta(*)$ as required contains $E \cap\left\{\delta<\mu^{+}\right.$: $\left.\operatorname{cf}(\delta)=\aleph_{0}\right\}$ for some club $E$ (in fact even contains $E$ ). So if $\mu \geq 2^{|T|}$, as $\left\{\operatorname{tp}^{\prime}(\overline{\mathbf{b}}): \overline{\mathbf{b}}\right.$ an infinite indiscernible sequence $\}$ has cardinality $\leq 2^{|T|}$ we are done.

Otherwise choose $J^{*}$ a linear order of cardinality $\mu$ isomorphic to its inverse, to $J^{*} \times \omega$ and to $J^{*} \times(\gamma+1)$ ordered lexicographically for every $\gamma \leq \mu$ hence for every $\gamma<\mu^{+}$, (e.g. note if $J^{* *}$ is dense with no first and last element and saturated (or special) of cardinality $>\mu$, then $J^{* *} \times \omega$ satisfies this and use the L.S. argument). So we can in 2.6 hence 2.8 use $I_{\ell}(\ell<4)$ such that $I_{\ell}^{\beta+1} \cong J^{*}$ for $\beta<\mu^{+}, \ell<4$. So $I_{0}^{\beta(*)}+I_{1}^{\beta(*)} \cong J^{*} \cong I_{2}^{\beta(*)}+I_{3}^{\beta(*)}$.

\subsection{Conclusion. In 2.9:}

(A) we can choose $J^{*}=\mu^{*}+\mu$ i.e. $\{0\} \times(\mu,>)+\{1\} \times(\mu,<)$

$(B)$ if $J$ is a linear order $(\neq \emptyset)$ of cardinality $\leq \mu$, we can use $J^{*}=\left(\mu^{*}+\mu\right) \times J$ ordered lexicographically

$(C)$ we can change the conclusion of 2.9 to make it symmetrical between $J_{3}$ and $J_{1}$

$(D)$ we use only clause $(E)_{2}$ of 2.6 or we could use only clause $(E)_{1}$.

Proof. (A),(B) combine the proofs of 2.3 and 2.6 trying to contradict each formula, by bookkeeping trying for it enough times. 
We may look at it differently, part (2) is close in formulation to be a complement to $[$ Sh $715, \S 5]$.

\subsection{Conclusion. 1) Assume}

(a) $J=I \times J^{*}$ is ordered lexicographically, $J^{*}, \mu$ are as in 2.9, $I$ infinite

(b) $\left\langle\bar{b}_{t}: t \in J\right\rangle$ an indiscernible sequence, $\ell g\left(\bar{b}_{t}\right)=m$ or just $\ell g\left(\bar{b}_{t}\right)<\mu^{+}$

(c) for $s \in I$ let $\bar{c}_{s}$ be $\left\langle\bar{b}_{t}: t \in\{s\} \times J^{*}\right\rangle$, more exactly the concatanation of the sequences in $\bar{b}_{t}$ for $t \in\{s\} \times J^{*}$.

Then

$(\alpha)\left\langle\bar{c}_{s}: s \in I\right\rangle$ is an infinite indiscernible sequence

$(\beta)$ if $s_{0}<_{I} \ldots<_{I} s_{7}$ then there is $\bar{c}$ realizing $\operatorname{tp}\left(\bar{c}_{s_{2}}, \bigcup\left\{\bar{c}_{s_{\ell}}: \ell \leq 7, \ell \neq 2\right\}\right)$ such that $\operatorname{tp}\left(\bar{c}, \cup\left\{\bar{c}_{s_{\ell}}: \ell \leq 7, \ell \neq 2\right\}\right) \vdash \operatorname{tp}\left(\bar{c}_{s_{2}}, \bigcup\left\{\bar{c}_{s}: s_{0} \leq_{I} s \leq_{I} s_{1}\right.\right.$ or $s_{3} \leq_{I} s \leq_{I} s_{4}$ or $\left.\left.s_{6} \leq_{I} s \leq_{I} s_{7}\right\}\right)$

$(\gamma)$ similarly inverting the order (i.e. interchanging the roles of $s_{2}, s_{5}$ in clause $(\beta))$.

2) Assume the sequence $\left\langle\bar{c}_{s}: s \in I\right\rangle$ from part (1) satisfies $M \supseteq \bigcup\left\{\bar{c}_{s}: s \in I\right\}$ and $\left(I_{1}, I_{2}\right),\left(I_{3}, I_{4}\right)$ are Dedekind cuts of $I$, each of $I_{1},\left(I_{2}\right)^{*}, I_{3},\left(I_{4}\right)^{*}$ is non-empty of cofinality $\leq \mu$ and let $I^{+} \supseteq I, t_{2}, t_{5} \in I_{1}^{+}$realize the cuts $\left(I_{1}, I_{2}\right),\left(I_{3}, I_{4}\right)$ respectively and $\bar{c}_{t}$ for $t \in I^{+} \backslash I$ are such that $\left\langle\bar{c}_{t}: t \in I^{+}\right\rangle$is indiscernible (then for notational simplicity). Then

$\checkmark$ there is a sequence in $M$ realizing $\operatorname{tp}\left(\bar{c}_{t_{2}}, \bigcup\left\{\bar{c}_{s}: s \in I\right\}\right)$ iff there is a sequence in $M$ realizing $\operatorname{tp}\left(\bar{c}_{t_{5}}, \bigcup\left\{\bar{c}_{s}: s \in I\right\}\right)$.

Concluding Remark. There is a gap between [Sh 715, 5.11=np5.5] and the results in $\S 2$, some light is thrown by

2.12 Claim. In [Sh 715, 5.11=np5.5]; we can omit the demand $\mathrm{cf}\left(\operatorname{Dom}\left(\overline{\mathbf{a}}^{\zeta}\right)\right) \geq \kappa_{1}$ $(=$ clause $(f)$ there $)$ if we add $\zeta<\zeta^{*} \Rightarrow\left(\theta_{\zeta}^{1}\right)^{+}=\lambda$.

Proof. By the omitting type argument.

2.13 Question: Assume:

(a) $\left\langle\left(N_{i}, M_{i}\right): i \leq \kappa\right\rangle$ is $\prec$-increasing (as pairs), $M_{i+1}, N_{i+1}$ are $\lambda_{i}^{+}$-saturated, $\left\|N_{i}\right\| \leq \lambda_{i},\left\langle\lambda_{i}: i<\kappa\right\rangle$ increasing, $\kappa<\lambda_{0}$

(b) $p(\bar{x})$ is a partial type over $N_{0} \cup M_{\kappa}$ of cardinality $\leq \lambda_{0}$. 
1) Does $p(\bar{x})$ have a $\lambda_{0}^{+}$-isolated extension?

2) Does this help to clarify DOP?

3) Does this help to clarify "if any $M$ is a benign set" (see [BBSh 815]).

2.14 Claim. Assume

(a) $M$ is $\lambda^{+}$-saturated

(b) $p(\bar{x})$ is a type of cardinality $\leq \kappa, \ell g(\bar{x}) \leq \kappa$

(c) $\operatorname{Dom}(p) \subseteq A \cup M,|A| \leq \kappa \leq \lambda$

(d) $B \subseteq M,|B| \leq \lambda$.

Then there is a type $q(\bar{x})$ over $A \cup M$ of cardinality $<\kappa$ and $r(\bar{x}) \in \mathbf{S}^{\ell g(\bar{x})}(A \cup B)$ such that

$$
\begin{aligned}
& p(\bar{x}) \subseteq q(\bar{x}) \\
& q(\bar{x}) \vdash r(\bar{x})
\end{aligned}
$$

Remark. This defines a natural quasi order (type definable) is it directed? 


\section{$\S 3$ STRONGLY DEPENDENT THEORIES}

3.1 Context. $T$ complete first order, $\mathfrak{C}$ a monster model of $T$.

3.2 Definition. 1) $T$ is strongly ${ }^{1}$ dependent (we may omit the 1) if: there are no $\bar{\varphi}=\left\langle\varphi_{n}\left(\bar{x}, \bar{y}_{n}\right): n<\omega\right\rangle$ and $\left\langle\bar{a}_{\alpha}^{n}: n<\omega, \alpha<\lambda\right\rangle$ such that

(*) for every $\eta \in{ }^{\omega} \lambda$ the set $p_{\eta}=\left\{\varphi_{n}\left(\bar{x}, \bar{a}_{\alpha}^{n}\right)^{\mathrm{if}(\eta(n)=\alpha)}: \alpha<\lambda\right\}$ is consistent; so $\ell g\left(\bar{a}_{\alpha}^{n}\right)=\ell g\left(\bar{y}_{n}\right)$.

2) $T$ is strongly stable if it is stable and strongly dependent.

$3) \kappa_{\text {ict }}(T)$ is the first $\kappa$ such that there is no $\bar{\varphi}=\left\langle\varphi_{\alpha}\left(\bar{x}, \bar{y}_{\alpha}\right): \alpha<\kappa\right\rangle$ satisfying the parallel of part (1), in this case we say that $\bar{\varphi}$ witnesses $\kappa<\kappa_{\text {ict }}(T)$ and let $m(\bar{\varphi})=\ell g(\bar{x})$.

3.3 Claim. 1) If $T$ is superstable, then $T$ is strongly dependent.

2) If $T$ is strongly dependent, then $T$ is dependent.

3) There are stable $T$ which are not strongly dependent.

4) There are stable not superstable $T$ which are strongly dependent.

5) There are unstable strongly dependent theories.

6) The theory of real closed fields is strongly dependent; moreover every o-minimal (complete first-order) $T$ is strongly dependent.

7) If $T$ is stable then $\kappa_{\text {ict }}(T) \leq \kappa(T)$.

8) If $T$ is dependent then we may add, in 3.2(1)

$(* *)$ for each $n<\omega$ for some $k_{n}$ any $k_{n}$ of the formulas $\left\{\varphi_{n}\left(\bar{x}, \bar{a}_{\alpha}^{n}\right): \alpha<\lambda\right\}$ are contradictory.

Proof. 1),2),7),8) Easy.

3) E.g. $T=\operatorname{Th}\left({ }^{\omega} \omega, E_{n}^{1}\right)_{n<\omega}$ where $\eta E_{n}^{1} \nu \Leftrightarrow \eta(n)=\nu(n)$ and use $\varphi_{n}\left(x, y_{n}\right)=$ $x E_{n}^{1} y_{n}$ for $n<\omega$.

4) E.g., $T=\operatorname{Th}\left({ }^{\omega} \omega, E_{n}^{2}\right)_{n<\omega}$ where $\left(\eta E_{n} \nu\right) \Leftrightarrow(\eta\lceil n=\nu \uparrow n)$.

5) E.g., $T=\operatorname{Th}(\mathbb{Q},<)$, the theory of dense linear orders with no first and no last element.

6) For simplicity we use $\bar{x}=\langle x\rangle$, (justified in [Sh 863, Observation,1.7](1)). Assume $\left\langle\varphi_{n}\left(x, \bar{y}_{n}\right): n<\omega\right\rangle$ and $\left\langle\bar{a}_{\alpha}^{n}: \alpha<\lambda\right\rangle$ are as in Definition 3.2. Clearly we can replace $\varphi_{n}\left(x, \bar{y}_{n}\right), \bar{a}_{\alpha}^{n}$ by $\varphi^{\prime}\left(x, \bar{y}_{n}^{\prime}\right), \bar{b}_{\alpha}^{n}$ when $\bar{y}_{n} \unlhd \bar{y}_{n}^{\prime}, \bar{a}_{\alpha}^{n} \unlhd \bar{b}_{\alpha}^{n}$ and $\varphi_{n}\left(x, \bar{a}_{\alpha}^{n}\right) \equiv \varphi_{n}^{\prime}\left(x, \bar{b}_{\alpha}^{n}\right)$. Also we can restrict ourselves to $\left\langle\bar{a}_{\alpha}^{n}: n<\omega, \alpha \in u_{n}\right\rangle$ where $u_{n} \subseteq \lambda$ is infinite for $n<\omega$. Hence by the elimination of quantifiers and density of the linear order 
without loss of generality $\varphi_{n}\left(x, \bar{y}_{n}\right)=\left(\varphi_{\eta, 1}\left(x, \bar{y}_{n}\right) \vee \varphi_{n, 2}\left(x, \bar{y}_{n}\right)\right) \wedge \varphi_{n, 3}\left(\bar{y}_{n}\right)$ where (without loss of generality $\bar{y}_{n}=\left\langle y_{\ell}: \ell=0, \ldots, k(n)\right\rangle, u(n, 1) \subseteq\{0, \ldots, k(n)-$ $1\}, u(n, 2) \subseteq\{0, \ldots, k(n)-1\}$

$$
\begin{gathered}
\varphi_{n, 1}\left(x, \bar{y}_{n}\right)=\bigvee_{\ell \in u(n, 1)}\left(y_{n, \ell}<x<y_{n, \ell+1}\right) \\
\varphi_{n, 2}\left(x, \bar{y}_{n}\right)=\bigvee_{\ell \in u(n, 2)} x=y_{n, \ell}
\end{gathered}
$$

and

$$
\varphi_{n, 3}(\bar{y})=\bigwedge_{\ell<k(n)} y_{n, \ell}<y_{n, \ell+1}
$$

As $\varphi_{n, 3}\left(\bar{a}_{\alpha}^{n}\right)$ is satisfied for every $n, \alpha$ as said above we can omit $\varphi_{n, 3}(\bar{y})$.

For each $\eta \in{ }^{\omega} \lambda, p_{\eta}$ is consistent (and $\eta \neq \nu \in{ }^{\omega} \lambda \Rightarrow p_{\eta}, p_{\nu}$ are contradictory), hence clearly each $p_{\eta}$ is not algebraic. From this it follows that $(*)$ of $3.2(1)$ is true also if we replace $\left\langle\varphi_{n}\left(x, \bar{y}_{n}\right): n<\omega\right\rangle$ by $\left\langle\varphi_{n, 1}\left(x, \bar{y}_{n}\right): n<\omega\right\rangle$. Also without loss of generality $\left\langle\bar{a}_{\alpha}^{n}: \alpha<\lambda\right\rangle$ is indiscernible over $\bigcup\left\{a_{\beta}^{m}: m \neq n, m<\omega\right.$ and $\beta<\lambda\}$. Now for some $\left\langle\ell_{n}: n<\omega\right\rangle \in \prod_{n<\omega} k(n)$, we can replace $\left\langle\varphi_{n}(x, \bar{y})\right.$ : $n<\omega\rangle$ by $\varphi_{n}^{\prime}(x, \bar{y})=y_{n, \ell_{n}}<x<y_{n, \ell_{n}+1}$. So without loss of generality $n<\omega \Rightarrow$ $k(n)=1, \ell_{n}=0, \bar{y}_{n}=\left(y_{n, 0}, y_{n, 1}\right)$.

Now $\left\langle\bar{a}_{\alpha}^{n}: \alpha<\lambda\right\rangle$ is an indiscernible sequence, and $\varphi_{n}\left(\mathfrak{C}, \bar{a}_{\alpha}^{n}\right)$ is the open convex set, actually an interval which $\bar{a}_{\alpha}^{n}$ define. So checking by cases (they are $a_{\alpha, 0}^{n}<$ $a_{\alpha, 1}^{n}<a_{\alpha+1,0}^{n}<a_{\alpha+1,1}^{n}, a_{\alpha, 0}^{n}<a_{\alpha+1,0}^{n}<a_{\alpha, 1}^{n}<a_{\alpha+1,1}^{n}, a_{\alpha+1,0}^{n}<a_{\alpha+1,1}^{n}<a_{\alpha, 0}^{n}<$ $a_{\alpha, 1}^{n}, a_{\alpha+1,0}^{n}<a_{\alpha, 0}^{n}<a_{\alpha+1,1}^{n}<a_{\alpha, 1}^{n}$ letting $p_{\beta}^{n}(x):=\left\{\varphi\left(x, \bar{a}_{\alpha}^{n}\right)^{\operatorname{if}(\alpha=\beta)}: \alpha<\lambda\right\}$ we note that it is a type such that $p_{\beta}^{n}(\mathfrak{C})$ is a convex set; obviously it is disjoint to $p_{\gamma}^{n}(\mathfrak{C})$ for $\gamma \in \lambda \backslash\{\beta\}$.

Clearly there are $\alpha \neq \beta<\lambda$ such that $p_{\alpha}^{0}(\mathfrak{C})<p_{\beta}^{0}(\mathfrak{C})$ and choose $a^{*}$ such that $p_{\alpha}^{0}(\mathfrak{C})<a^{*}<p_{\beta}^{0}(\mathfrak{C})$. Now for every $\gamma<\lambda$ we have $p_{\gamma}^{1}(\mathfrak{C}) \bigcap p_{\alpha}^{0}(\mathfrak{C}) \neq \emptyset$ and $p_{\gamma}^{1}(\mathfrak{C}) \bigcap p_{\beta}^{0}(\mathfrak{C}) \neq \emptyset$, i.e., $p_{0}^{1}(\mathfrak{C})$ is disjoint neither to $p_{\alpha}^{0}(\mathfrak{C})$ nor to $p_{\beta}^{0}(\mathfrak{C})$ (by the choice of $\left.\bar{\varphi},\left\langle\bar{a}_{\alpha}^{n}: n<\omega, \alpha<\lambda\right\rangle\right)$. As $p_{\gamma}^{1}(\mathfrak{C})$ is convex, by the choice of $a^{*}$ necessarily $a^{*} \in p_{\gamma}^{1}(\mathfrak{C})$. As $\gamma$ was any ordinal $<\lambda$ it follows that $a^{*} \in \bigcap\left\{p_{\gamma}^{1}(\mathfrak{C}): \gamma<\lambda\right\}$, clear contradiction. (In fact we get contradiction even if we use only $n=0,1$, see on this [Sh 863]). The o-minimal case holds by the same proof. 
3.4 Definition. 1) We say a pair of types $(p(x), q(\bar{y}))$ is a $\left(1=\aleph_{0}\right)$-pair of types (or $(p(\bar{x}), q(\bar{y}))$ satisfies $1=\aleph_{0}$ ) if there is a set $A$ such that: for every countable set $B \subseteq p(\mathfrak{C})$, there is an element $\bar{a} \in q(\mathfrak{C})$ satisfying $B \subseteq \operatorname{acl}(\{\bar{a}\} \cup A)$. We say $p(x)$ is a $\left(1=\aleph_{0}\right)$-type if this holds for some $q(\bar{y})$.

1A) If $A=\operatorname{Dom}(p)$ we add purely. We call $A$ a witness to $p(x)$ being a $\left(1=\aleph_{0}\right)$ type.

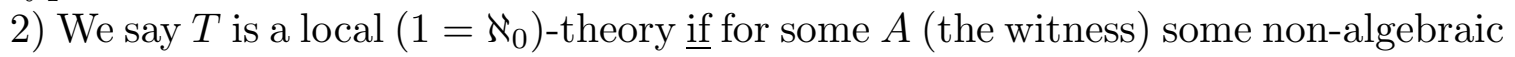
type $p$ over $A$ is a $\left(1=\aleph_{0}\right)$-type. If $A=\emptyset$ we say purely.

$2 \mathrm{~A})$ We say $T$ is a global $\left(1=\aleph_{0}\right)$-theory when the type $x=x$ is a $\left(1=\aleph_{0}\right)$-type.

3) We say that a pair $(p(x), q(\bar{y}))$ of types is a semi $\left(1=\aleph_{0}\right)$-pair of types $\underline{\text { if: }}$ for some set $A$ for every indiscernible sequence $\left\langle a_{n}: n<\omega\right\rangle$ over $A$ satisfying $n<\omega \Rightarrow \bar{a}_{n} \in p(\mathfrak{C})$ there is $\bar{a} \in q(\mathfrak{C})$ such that $\left\{\bar{a}_{n}: n<\omega\right\} \subseteq \operatorname{acl}(\bar{a} \cup A)$. We say $p(\bar{x})$ is semi $\left(\aleph_{0}=1\right)$-type if this holds for some $q(\bar{y})$.

4) We say that the pair $(p(x), q(\bar{y}))$ of types is a weakly $\left(1=\aleph_{0}\right)$-pair of types if there are $A \supseteq \operatorname{Dom}(p)$ and an infinite indiscernible sequence $\left\langle a_{n}: n<\omega\right\rangle$ over $A$ with each $a_{n}$ realizing $p$ such that for some $\bar{c} \in q(\mathfrak{C})$ we have $\left\{a_{n}: n<\omega\right\} \subseteq \operatorname{acl}(A \cup \bar{c})$. $5)$ We say $p(x)$ is semi/weakly $\left(\aleph_{0}=1\right)$-type if some pair $(q, p)$ is semi/weakly $\left(\aleph_{0}=1\right)$-pair of types.

6) In (3),(4) we let "purely", "witness" "local"; "global" be defined similarly.

7) Above we can allow $p=p(\bar{x}), \ell g(\bar{x})=m$.

3.5 Observation. 1) Every algebraic type $p(x)$ is a $\left(1=\aleph_{0}\right)$-type. If $p \subseteq q$ and $p$ is a $\left(1=\aleph_{0}\right)$-type then $q$ is an $\left(1=\aleph_{0}\right)$-type.

2) If $p(x)$ is a $\left(1=\aleph_{0}\right)$-type then $p(x)$ is a semi $\left(1=\aleph_{0}\right)$-type.

$3)$ If $p(x)$ is a semi $\left(1, \aleph_{0}\right)$-type then $p(x)$ is a weakly $\left(1=\aleph_{0}\right)$-type.

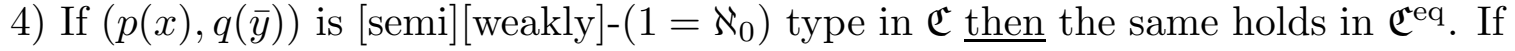

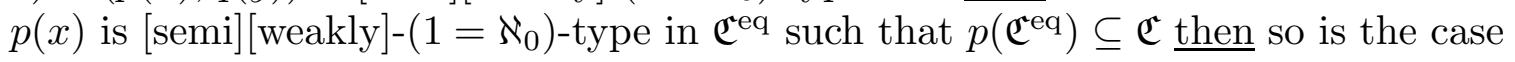
in $\mathfrak{C}$. We can also keep track of the witness.

$5)$ For some $T, T$ is not locally $\left(1=\aleph_{0}\right)$-theory but $T^{\text {eq }}$ is.

Proof. Easy.

3.6 Claim. 1) If $T$ is strongly dependent, then no non-algebraic type is a $\left(1=\aleph_{0}\right)$ type.

2) Moreover no non-algebraic type is a weakly $\left(1=\aleph_{0}\right)$-type.

Remark. We can weaken the assumption of 3.6 to: for some $\omega$-sequence of nonalgebraic types $\left\langle p_{n}(x): n<\omega\right\rangle$ over $A$, for every $\left\langle b_{n}: n<\omega\right\rangle \in \prod_{n<\omega} p_{n}(\mathfrak{C})$, for some $\bar{c}$ we have $\left\{b_{n}: n<\omega\right\} \subseteq c \ell(A \cup \bar{c})$. 
Proof. Let $\lambda>|T|^{+}$. Assume toward contradiction that $p(x)$ is a non-algebraic $\left(1=\aleph_{0}\right)$-type and $A$ a witness for it. As $p(x)$ is not algebraic, we can find $\bar{b}^{n}=$ $\left\langle b_{\alpha}^{n}: \alpha<\lambda\right\rangle$ for $n<\omega$ such that

$(*)_{1} b_{\alpha}^{n}$ realizes $p$

$(*)_{2} b_{\alpha}^{n} \neq b_{\beta}^{n}$ for $\alpha<\beta<\lambda, n<\omega$

$(*)_{3}\left\langle b_{\alpha}^{n}:(n, \alpha) \in \omega \times \lambda\right\rangle$ is an indiscernible sequence over $A$ where $\omega \times \lambda$ is ordered lexicographically.

Let $\bar{a} \in \omega>(\mathfrak{C})$ be such that $\left\{b_{0}^{n}: n<\omega\right\} \subseteq \operatorname{acl}(A \cup \bar{a})$ so for each $n$ we can find $k_{n}<\omega, \bar{c}_{n} \in \omega>A$ and a formula $\varphi_{n}(x, \bar{y}, \bar{z})$ such that $\mathfrak{C} \models \varphi\left(b_{0}^{n}, \bar{a}, \bar{c}_{n}\right) \quad \&$ $\left(\exists \leq k_{n} x\right) \varphi\left(x, \bar{a}, \bar{c}_{n}\right)$. By omitting some $b_{\alpha}^{n}$ 's we have $(n, \alpha) \in \omega \times \lambda \backslash\{(m, \omega): m<$ $\omega\} \Rightarrow \mathfrak{C} \models \neg \varphi_{n}\left[b_{\alpha}^{n}, \bar{a}, \bar{c}_{n}\right]$.

Let $\bar{a}_{\alpha}^{n}=\left\langle b_{\alpha}^{n}\right\rangle^{\wedge} \bar{c}_{n}$ and $\varphi_{n}$ have already been chosen.

Now check Definition 3.2.

3.7 Definition. 1) We say $T$ is $\operatorname{strongly}^{2}$ (or strongly ${ }^{+}$) dependent when: there is no sequence $\left\langle\varphi_{n}\left(\bar{x}, \bar{y}_{0}, \ldots, \bar{y}_{n}\right): n<\omega\right\rangle$ and $\bar{a}_{\alpha}^{n} \in \ell g\left(y_{n}\right) \mathfrak{C}$ for $n<\omega, \alpha<\lambda$ (any infinite $\lambda$ ) such that for every $\eta \in{ }^{\omega} \lambda$ the set $\left\{\varphi_{n}\left(\bar{x}, \bar{a}_{\eta(0)}^{0}, \ldots, a_{\eta(n-1)}^{n-1}, a_{\alpha}^{n}\right)^{\text {if }(\alpha=\eta(n))}\right.$ : $n<\omega, \alpha<\lambda\}$ is consistent.

2) Let $\ell \in\{1,2\}$. We say that $T$ is strongly ${ }^{\ell, *}$ dependent when: if $\left\langle\overline{\mathbf{a}}_{t}: t \in I\right\rangle$ is an indiscernible sequence over $A, t \in I \Rightarrow \ell g\left(\overline{\mathbf{a}}_{t}\right)=\alpha$ (so constant but not necessarily finite) and $m<\omega$ and $\bar{b}_{n} \in{ }^{m} \mathfrak{C}$ for $n<\omega,\left\langle\bar{b}_{n}: n<\omega\right\rangle$ is an indiscernible sequence over $A \cup\left\{\overline{\mathbf{a}}_{t}: t \in I\right\}$ then we can divide $I$ to finitely many convex sets $\left\langle I_{m}: m<k\right\rangle$ such that for each $m<k,\left\langle\overline{\mathbf{a}}_{t}: t \in I_{m}\right\rangle$ is an indiscernible sequence over $\cup\left\{\bar{b}_{\alpha}: \alpha<\omega\right\} \cup A \cup\left\{\bar{a}_{s}: s \in I \backslash I_{m}\right.$ and $\left.\ell=2\right\}$.

3) $T$ is strongly ${ }^{\ell}$ stable (or strongly ${ }^{\ell, *}$ stable) when it is strongly ${ }^{\ell}$ dependent (or strongly ${ }^{\ell, *}$ dependent) and stable.

3.8 Claim. If $T$ is strongly ${ }^{+}$dependent then:

$\circledast_{1}$ for any $A \subseteq \mathfrak{C}$, infinite complete linear order $I$ and indiscernible sequence $\left\langle\bar{a}_{t}: t \in I\right\rangle$ over $A, \lg \left(\bar{a}_{t}\right)$ possibly infinite, for any finite $B \subseteq \mathfrak{C}$, there is a finite $w \subseteq I$ such that: if $J$ is a convex subset of $I$ disjoint to $w$ then $\left\langle\bar{a}_{t}: t \in J\right\rangle$ is indiscernible over $A \cup B \cup\left\{\bar{a}_{s}: s \in I \backslash J\right\}$

$\circledast_{2}$ for any set $A \subseteq \mathfrak{C}$ of cardinality $\lambda$ and infinite linear orders $I_{\alpha}$ for $\alpha<\lambda$ and $\bar{a}_{t}^{\alpha}\left(t \in I_{\alpha}, \alpha<\lambda\right)$ such that $\left\langle\bar{a}_{t}^{\alpha}: t \in I_{\alpha}\right\rangle$ is an indiscernible sequence over $A \cup\left\{\bar{a}_{s}^{\beta}: \beta \in \lambda \backslash\{\alpha\}, s \in I_{\beta}\right\}$ and finite $B \subseteq \mathfrak{C}$ there is a finite $u \subseteq \lambda$ and $w_{\alpha} \in\left[I_{\alpha}\right]^{<\aleph_{0}}$ for $\alpha \in u$ such that: if $\bar{J}=\left\langle J_{\alpha}: \alpha<\lambda\right\rangle, J_{\alpha}$ is a convex subset of $I_{\alpha}$, disjoint to $w_{\alpha}$ when $\alpha \in u$ then $\left\langle\bar{a}_{t}^{\alpha}: t \in J_{\alpha}\right\rangle$ is indiscernible over $A \cup B \cup\left\{\bar{a}_{s}^{\beta}: \beta \in \lambda \backslash\{\alpha\}, s \in J_{\beta}\right\}$ for every $\alpha<\lambda$. 
Proof. See this (and more) [Sh 863, §2].

3.9 Definition. 1) We say that $\vartheta\left(x_{1}, x_{2} ; \bar{c}\right)$ is a finite-to-finite function from $\varphi_{1}\left(\mathfrak{C}, \bar{a}_{1}\right)$ onto $\varphi_{2}\left(\mathfrak{C}, \bar{a}_{2}\right)$ when:

(a) if $b_{2} \in \varphi_{2}\left(\mathfrak{C}, a_{2}\right)$ then the set $\left\{x: \vartheta\left(x, b_{2}, \bar{c}\right) \wedge \varphi_{1}\left(x, \bar{a}_{1}\right)\right\}$ satisfies:

(i) it is finite but

(ii) it is not empty except for finitely many such $b_{2}$ 's

(b) if $b_{1} \in \varphi_{1}\left(\mathfrak{C}, \bar{a}_{1}\right)$ then the set $\left\{x: \vartheta\left(b_{1}, x, \bar{c}\right) \wedge \varphi_{2}\left(x, \bar{a}_{2}\right)\right\}$ satisfies:

(i) it is finite but:

(ii) it is not empty except for finitely many such $b_{1}$ 's.

2) If we place "onto $\varphi_{2}\left(\mathfrak{C}, \bar{a}_{2}\right)$ " by "into $\varphi_{2}\left(\mathfrak{C}, \bar{a}_{1}\right)$ " we mean that we require above only clauses $(a)(i),(b)(i),(i i)$.

3) We can replace $\varphi_{1}\left(x, \bar{a}_{1}\right), \varphi_{2}\left(x, \bar{a}_{2}\right)$ above by types.

3.10 Claim. If $T$ is strongly ${ }^{+}$dependent then the following are impossible:

$(S t)_{1}$ for some $\varphi(x, \bar{a})$

(a) $\varphi(x, \bar{a})$ is not algebraic

(b) $E$ is a definable equivalence relation (in $\mathfrak{C}$ by a first order formula possibly with parameters) with domain $\subseteq \varphi(\mathfrak{C}, \bar{a})$ and infinitely many equivalence classes

(c) there is a formula $\vartheta(x, y, \bar{z})$ such that for every $b \in \operatorname{Dom}(E)$ for some $\bar{c}$, the formula $\vartheta(x, y ; \bar{c})$ is a finite to finite map from $\varphi(\mathfrak{C}, \bar{a})$ into $b / E$;

$(S t)_{2}$ for some formulas $\varphi(x), x E y, \vartheta(x, y, \bar{z})$ possibly with parameters we have:

(a) $\varphi(x)$ is non-algebraic

(b) $x E y \rightarrow \varphi(x) \wedge \varphi(y)$

(c) for uncountably many $c \in \varphi(\mathfrak{C})$ for some $\bar{d}$ the formula $\vartheta(x, y ; \bar{d})$ is a finite to finite function from $\varphi(x)$ into $x E c$

(d) for some $k<\omega$, if $b_{1}, \ldots, b_{k} \in \varphi(\mathfrak{C})$ are pairwise distinct then $\bigwedge_{\ell=1}^{k} x E b_{\ell}$ is algebraic 
$(S t)_{3}$ similarly with $\varphi(x, \bar{a})$ replaced by a type, as well as $x$ Ey replacing uncountable by cardinality of $\bar{\kappa}$ (and $x, y, z$ are replaced by $m$-tuples).

Proof. The proof for $(S t)_{1}$ is a special case of the proof of $(S t)_{2}$ and the proof for $(S t)_{3}$ is similar but choosing $k^{*}$ and $r(x)$ we should use for subtypes of $\varphi(x), x \in$ $y, \vartheta(x, y, \bar{z})$. So it is enough:

Proof of " $(S t)_{2}$ is impossible":

Without loss of generality in clause (c) of $(S t)_{2}$ we have $\langle c\rangle \triangleleft \bar{d}$ and let $\ell g(\bar{d})=j$, i.e., $\vartheta=\vartheta(x, y, \bar{z}), \ell g(\bar{z})=j$; also let $\bar{z}^{n}=\left\langle z_{n, 0}, \ldots, z_{n, j-1}\right\rangle$.

Clearly there is $k^{*}$ such that

$\nabla_{1}$ for some uncountably $C \subseteq \varphi(\mathfrak{C})$ for every $c \in C$ for some $\bar{d}_{c} \in{ }^{j} \mathfrak{C}$ without loss of generality $\langle c\rangle \triangleleft$ $\bar{d}_{c}$ and $\vartheta\left(x, y, \bar{d}_{c}\right)$ is a finite to finite map from $\varphi(\mathfrak{C})$ into $x E c$ and the size of the finite sets (see Definition 3.9) is $<k^{*}$ replacing the type by fixed finite sub-types;

$\square_{2}$ moreover $C=r(\mathfrak{C})$ for some non-algebraic $r(x)$;

$\square_{3} k^{*}$ can serve as $k$ in clause $(\mathrm{d})$ of $(S t)_{2}$.

Let $\bar{z}_{n}=\bar{z}^{0 \wedge} \ldots{ }^{\wedge} \bar{z}^{n-1}$. We shall now define by induction on $n<\omega$ formulas $\varphi_{n}\left(x, \bar{z}_{n}\right)$ and $\vartheta_{n}\left(x_{1}, x_{2}, \bar{z}_{n}\right)$ also written $\varphi_{\bar{z}_{n}}^{n}(x), \vartheta_{\bar{z}_{n}}^{n}\left(x_{1}, x_{2}\right)$.

Case 1: $n=0$.

So $\left(\bar{z}_{n}=<>\right.$, and $\varphi_{n}(x)=\varphi(x)$ and $\vartheta_{n}\left(x_{1}, x_{2}\right)=\left(x_{1}=x_{2}\right)$.

Case 2: $n=m+1$.

Let $\varphi_{\bar{z}_{n}}^{n}(x):=\varphi_{\bar{z}_{m}}^{m}(x) \&\left(\exists x^{\prime}\right)\left[x^{\prime} E z_{m} \wedge \vartheta_{\bar{z}_{m}}^{m}\left(x^{\prime}, x\right)\right]$ and $\vartheta_{\bar{z}_{n}}^{n}\left(x_{1}, x_{2}\right):=\varphi_{\bar{z}_{m}}^{m}\left(x_{2}\right) \quad \&$ $\varphi\left(x_{1}\right) \&\left(\exists x^{\prime}\right)\left[\vartheta\left(x_{1}, x^{\prime}, \bar{z}^{m}\right) \wedge \vartheta_{\bar{z}_{m}}^{m}\left(x^{\prime}, x_{2}\right) \wedge x^{\prime} E z_{m}\right]$.

We now prove by induction on $n$ that:

$(*)_{n}$ if $\bar{c}=\left\langle c_{\ell}: \ell<n\right\rangle$ and $c_{\ell} \in C \backslash a c \ell\left\{c_{k}: k<\ell\right\}$ for $\ell<n$ (so $\vartheta\left(x, y, \bar{d}_{c_{\ell}}\right)$ is a finite to finite function from $\varphi(x)$ into $x E c_{\ell}$ for $\left.\ell<n\right)$ and $\bar{d}=$ $\bar{d}_{c_{0}} \hat{d}_{c_{1}} \wedge \ldots \bar{d}_{c_{n-1}}$ then

( $\alpha) \varphi_{\bar{d}}^{n}(\mathfrak{C})$ is an infinite subset of $\varphi(\mathfrak{C})$

( $\beta) \vartheta_{\bar{d}}^{n}\left(x_{1}, x_{2}\right)$ is a finite to finite function from a co-finite subset of $\varphi(\mathfrak{C})$ into a subset of $\varphi_{\bar{d}}^{n}\left(\mathfrak{C}^{\mathbf{c}}\right)$

$(\gamma)$ if $n=m+1$ and $e \in \varphi_{\bar{d}}^{n}(\mathfrak{C})$ then $\left(\exists c^{\prime} \in \operatorname{ac\ell }(\bar{c}\lceil m \cup\{e\}))\left[c^{\prime} E c_{m}\right]\right.$

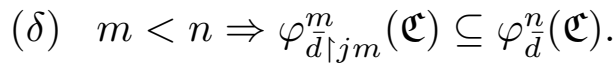


This is straight. Let $I$ be a linear order such that any interval has $<|T|$ members.

By $\bullet_{2}, \bullet_{3}$ there are $c_{t} \in C$ for $t \in I$ pairwise distinct, let $\bar{d}_{t}=\bar{d}_{c_{t}}$ so $\theta\left(x, y, \bar{d}_{t}\right)$ is a finite to finite function from $\varphi(x)$ into $x E c_{t}$ such that $\left\langle\bar{d}_{t}: t \in I\right\rangle$ is an indisernible sequence (e.g. use $\square$ above).

Now for every $<_{I}$-increasing sequence $\bar{t}=\left\langle t_{n}: n<\omega\right\rangle$ we consider $\bar{c}_{\bar{t}}^{n}=\bar{c}_{\bar{t} \uparrow n}^{n}=$ $\bar{d}_{t_{0}} \wedge \ldots \bar{d}_{t_{n-1}}$ and $p_{\bar{t}}=\left\{\varphi_{\bar{c}_{\bar{t} \mid n}^{n}}^{n}(x): n<\omega\right\}$.

Now

$\circledast_{1}$ for $\bar{t}$ as above $p_{\bar{t}}$ is consistent.

[Why? By $(*)_{n}(\alpha)$ there is an element $e \in \varphi_{\bar{c}_{\bar{t}}^{n}}^{n}(\mathfrak{C})$, by $(*)_{n}(\delta)$ the element $e$ satisfies $\left\{\varphi_{\bar{c}_{\bar{t} \uparrow m}^{m}}(x): m \leq n\right\}$. As this holds for every $n$, the set $p_{\bar{t}}=\left\{\varphi_{\bar{d}_{\bar{t} \mid n}^{n}}^{n}(x): n<\omega\right\}$ is finitely satisfiable as required.]

$\circledast_{2}$ if $e$ realizes $p_{\bar{t}}$ then for every $n$ there is an element $e^{\prime}$ algebraic over $\left\{e, \bar{d}_{t_{0}}, \ldots, \bar{d}_{t_{n-1}}\right\}$ such that $e^{\prime} E b_{t_{n}}^{1}$.

$\left[\right.$ Why? By $\left.(*)_{n}(\gamma) \cdot\right]$

$\circledast_{3}$ if $e$ realizes $p_{\bar{t}}$ then for every $n$ the set $\left\{s \in I\right.$ : there is $e^{\prime}$ algebraic over $\left\{e, \bar{d}_{t_{0}}^{1}, \ldots, \bar{d}_{t_{n-1}}^{1}\right\}$ such that $\left.e^{\prime} E c_{s}^{1}\right\}$ has $\leq|T|$ members.

[Why? There are $\leq|T|$ such $e^{\prime}$ and for each $e^{\prime}$ by clause (d) of $(\mathrm{St})_{2}$ there are only finitely many such $s \in I$ (if we phrase it more carefully we get that there are $<k_{n}(<\omega)$ many members $)$.]

This is more than enough to show $T$ is not strongly ${ }^{+}$dependent. $\quad \square 3.10$

Discussion: We may phrase 3.10 for ideals of small formulas.

3.11 Claim. If $T$ is strongly ${ }^{1}$ dependent and $\ell=1,2,3,4$, then the statement $\circledast \ell$ below is impossible where:

$\circledast_{1}(a)\left\langle\bar{a}_{\alpha}: \alpha<\lambda\right\rangle$ is an indiscernible sequence over $A$

(b) $u_{n} \subseteq \lambda$ is finite, [non-empty] with $\left\langle u_{n}: n<\omega\right\rangle$ having pairwise disjoint convex hull

(c) $\bar{b} \in \omega>\mathfrak{C}$

(d) for each $n$ for some $\alpha_{n}, k$ and $t_{n(0)}^{\mathbf{t}}<\ldots<t_{n(k-1)}^{\mathbf{t}} \in u_{n}$ for $\mathbf{t} \in\{$ false,truth $\}$ and $\bar{c}_{n} \in \omega>A$ and $\varphi$ we have $\mathfrak{C} \models \varphi\left(\bar{c}, a_{t_{n(0)}^{\mathrm{t}}}, \ldots, a_{t_{n(k-1)}^{\mathrm{t}}}, \bar{c}_{n}\right)^{\mathbf{t}}$ for both values of $\mathbf{t}$

$\circledast_{2} \quad$ like $\circledast_{1}$ but allows $\bar{a}_{\alpha}$ to be infinite 
$\circledast_{3}(a)\left\langle\bar{a}_{\alpha}^{n}: \alpha<\lambda\right\rangle$ is an indiscernible sequence over $A \cup\left\{\bar{a}_{\beta}^{m}: m<\omega, m \neq\right.$ $n$ and $\beta<\lambda\}$

(b) $\bar{a}_{\alpha}^{n} \neq \bar{a}_{\alpha+1}^{n}$

(c) some $\bar{a} \in \mathfrak{C}$ satisfies $n<\omega \Rightarrow \operatorname{acl}(A \cup\{a\}) \cap\left\{\bar{a}_{\alpha}^{n}: \alpha<\lambda\right\} \neq \emptyset$

$\circledast_{4} \quad$ like $\circledast_{3}$ but replace clause $(c)$ by

$(c)^{\prime}$ for some $\bar{a} \in \mathfrak{C}$ for every $n$ the sequence $\left\langle\bar{a}_{\alpha}^{n}: \alpha<\lambda\right\rangle$ is not an indiscernible sequence over $A \cup \bar{a}$.

Proof. Similar to the previous ones.

3.12 Discussion: 1) We have asked: show that the theory of the $p$-adic field is strongly dependent.

Udi Hrushovski has noted that the criterion $(\mathrm{St})_{2}$ from 3.10 from this section apply so $T$ is not strongly ${ }^{2}$ dependent. Namely take the following equivalence relation on $\mathbb{Z}_{p}: \operatorname{val}(x-y) \geq \operatorname{val}(c)$, where $c$ is some fixed element with infinite valuation. Given $x$, the map $y \mapsto(x+c y)$ is a bijection between $\mathbb{Z}_{p}$ and the class. 2) By [Sh 863] this theory is strongly ${ }^{1}$ dependent.

3) Onshuus shows that also the theory of the field of the reals is not strongly ${ }^{2}$ dependent (e.g. though Claim 3.10 does not apply but its proof works (using pairwise not too near $\bar{b}$ 's, in general just an uncountable set of $\bar{b}$ 's).

4) In [Sh 863] we prove reasonable existence of indiscernibles for strongly dependent $T$ (and in 3.2 we can use the case $\ell g(\bar{x})=1$ ).

3.13 Claim. Assume $x=1$ or $x=2$ or $x=1^{*}$ or $x=2^{*}$.

1) If $M \prec \mathfrak{C}, A \subseteq \mathfrak{C} \underline{\text { then }}$ (the complete first order) $T h\left(\mathfrak{B}_{M, M, A}\right.$ from 1.10(2) is strongly ${ }^{x}$ dependent iff $T$ is strongly ${ }^{x}$ dependent. If $T$ is dependent this theory is equal to $T_{M, A}^{*}$, see 1.10(4), 1.12(4).

2) $\kappa_{\text {ict }}(T)=\kappa_{\text {ict }}\left(\operatorname{Th}\left(\mathfrak{B}_{M, M A}\right)\right)$ if $M \prec \mathfrak{C}, A \subseteq \mathfrak{C}$

3) If $T_{1} \subseteq T_{2}$ are complete first order theories (so $\tau\left(T_{1}\right) \subseteq \tau\left(T_{2}\right)$ ) then

(a) if $T_{2}$ is strongly ${ }^{x}$ dependent then so is $T_{1}$

(b) $\kappa_{\text {ict }}\left(T_{1}\right) \leq \kappa_{\text {ict }}\left(T_{2}\right)$.

4) If $T_{1} \subseteq T_{2}$ are complete first order and $\tau\left(T_{2}\right) \backslash \tau\left(T_{1}\right)$ consist of individual constants only then

( $\alpha) T_{2}$ is strongly ${ }^{x}$ dependent iff $T_{1}$ is strongly ${ }^{x}$ dependent

( $\beta) \kappa_{\mathrm{ict}}\left(T_{1}\right)=\kappa_{\mathrm{ict}}\left(T_{2}\right)$. 
5) For $T$ is strongly ${ }^{x}$ dependent iff $T^{\mathrm{eq}}$ is strongly ${ }^{x}$ dependent; similarly for strongly ${ }^{\ell, *}$.

6) $\kappa_{\mathrm{ict}}(T)=\kappa_{\mathrm{ict}}\left(T^{\mathrm{eq}}\right)$.

Proof. Easy. 


\section{$\S 4$ Definable Groups}

\subsection{Context.}

(a) $T$ is a first order complete theory

(b) $\mathfrak{C}$ is a monster model of $T$.

We try here to generalize the theorem on the existence of commutative infinite subgroups for stable $T$ to dependent $T$. Theorems on definable groups in a monster $\mathfrak{C}, \operatorname{Th}(\mathfrak{C})$ stable, are well known.

4.2 Definition. 1) We say $G$ is a type-definable group (in $\mathfrak{C})$ if $G=(p, *$, inv) $=$ $\left(p^{G}, *^{G}\right.$, inv $\left.^{G}\right)$ where

(a) $p=p(x)$ is a type

$(b) *$ is a two-place function on $\mathfrak{C}$, possibly partial, definable (in $\mathfrak{C}$ ), we normally write $a b$ instead of $a * b$ or $*(a, b)$

(c) $(p(\mathfrak{C}), *)$ is a group, we write $x \in G$ for $x \in p(\mathfrak{C})$;

$(d)$ inv $^{G}$ is a (partial) unary function, definable (in $\mathfrak{C}$ ), which on $p(\mathfrak{C})$ is the inverse, so if no confusion arises we shall write $(x)^{-1}$ for $\operatorname{inv}(x)$.

1A) We let $B_{2}^{G}$ be the set of parameters appearing in $p^{G}$; let $B^{G}$ be the set of parameters appearing in $p^{G}$ or in the definition of $*$ or of inv $^{G}$.

2) We say $G$ is a definable group if $p(x)$ is a formula, i.e., a singleton.

3) We say $G$ is an almost type definable group if $p(x)$ is replaced by $\bar{p}=\left\langle p_{i}(x)\right.$ : $i<\delta\rangle, p_{i}(\mathfrak{C})$ increasing with $i$ and $\bar{p}(\mathfrak{C})$ is defined as $\cup\left\{p_{i}(\mathfrak{C}): i<\delta\right\}$.

Remark. Of course, we can use $p(\bar{x})$ and/or work in $\mathfrak{C}^{\mathrm{eq}}$.

4.3 Claim. Assume

(a) $T$ is dependent

(b) $G$ is a definable group in $\mathfrak{C}$ or just type-definable

(c) $A \subseteq G$ is a set of pairwise commuting elements, $D$ a non-principal ultrafilter on $A$ or just

$(c)^{-} A \subseteq G, D$ a non-principal ultrafilter on $A$ such that $\left(\forall^{D} a_{1}\right)\left(\forall^{D} a_{2}\right)\left(a_{1} a_{2}=\right.$ $\left.a_{2} a_{1}\right)$, where $\forall^{D} x \varphi(x, \bar{a})$ means $\{b \in \operatorname{Dom}(D): \mathfrak{C} \models \varphi[b, \bar{a}]\} \in D$. 
Then there is a formula $\varphi(x, \bar{a})$ such that:

( $\alpha) \varphi(x, \bar{a}) \in \operatorname{Av}(\bar{a}, D)$

( $\beta) G \cap \varphi(\mathfrak{C}, \bar{a})$ is an abelian subgroup of $G$

$(\gamma) \bar{a} \subseteq A \cup B^{G} \cup\left\{c: c\right.$ realizes $\left.\operatorname{Av}\left(A \cup B_{2}^{G}, D\right)\right\}$.

4.4 Remark. 1) If $D$ is a principal ultrafilter say $\left\{a^{*}\right\} \in D$ then $\varphi(x, \bar{a})$ is essentially $\mathrm{Cm}_{G}\left(\mathrm{Cm}_{G}\left(a^{*}\right)\right)$ so no new point, recalling $\left(\mathrm{Cm}_{G}(A)=\{x \in A: x\right.$ commutes with every $a \in A\}$.

2) If $D$ is a non-principal ultrafilter, then necessarily $\varphi(x, \bar{a})$ is not algebraic as it belongs to $\operatorname{Av}(\bar{a}, D)$.

Proof. We try to choose $a_{n}, b_{n}$ by induction on $n<\omega$ such that:

(i) $a_{n}, b_{n}$ realizes $p_{n}(x):=\operatorname{Av}\left(A_{n}, D\right)$ where $A_{n}=A \cup B^{G} \cup\left\{a_{k}, b_{k}: k<n\right\}$ so as $A \in D, A \subseteq G$ necessarily $p^{G}(x) \subseteq p_{n}(x)$

(ii) $a_{n}, b_{n}$ does not commute (in $G$, they are in $G$ because $p^{G}(x) \subseteq p_{n}$ ).

Case 1: We succeed.

Assume $n<m<\omega, c^{\prime} \in\left\{a_{n}, b_{n}\right\}$ and $c^{\prime \prime} \in\left\{a_{m}, b_{m}\right\}$ clearly $c^{\prime}, c^{\prime \prime}$ are in $G$. Now we shall show that they commute because $c^{\prime \prime}$ realizes $\operatorname{Av}\left(A \cup B^{G} \cup\left\{c^{\prime}\right\}, D\right)$ and $c^{\prime}$ realizes $\operatorname{Av}\left(A \cup B_{2}^{G}, D\right)$ recalling either assumption (c) about commuting in $A$ or assumption $(c)^{-}$. Hence if $k<\omega, n_{0}<\ldots<n_{k-1}<\omega$ and $n<\omega$ then $c:=b_{n_{0}} b_{n_{1}} \ldots b_{n_{k-1}}$ satisfies: $c, a_{n}$ commute iff $n \notin\left\{n_{0}, \ldots, n_{k-1}\right\}$, so $\varphi(x, y)=$ $[x y=y x]$ has the independence property contradicting assumption (a).

Case 2: We are stuck at $n<\omega$.

So $p_{n}(x) \cup p_{n}(y) \vdash(x y=y x)$, hence there is a formula $\psi\left(x, \bar{a}^{*}\right) \in \operatorname{Av}\left(A_{n}, D\right)$ such that

$(*)_{1} \psi\left(x, \bar{a}^{*}\right) \wedge \psi\left(y, \bar{a}^{*}\right) \vdash x y=y x$ (so both products are well defined).

Let $p^{G}(x)=\{\vartheta(x, \bar{a})\}$ or just $p(x) \vdash \vartheta(x, \bar{a})$ and $\theta(x, \bar{a}) \wedge \theta(y, \bar{a}) \vdash(x y$ well defined $)$; without loss of generality $\bar{a} \unlhd \bar{a}^{*}$ and $\psi\left(x, \bar{a}^{*}\right) \vdash \vartheta(x, \bar{a})$ and let $\vartheta^{*}\left(x, \bar{a}^{*}\right)=\vartheta(x, \bar{a}) \wedge$ $(\forall y)\left[\psi\left(y, \bar{a}^{*}\right) \rightarrow y x=x y\right.$ (so both well defined)]. So $\psi\left(x, \bar{a}^{*}\right) \vdash \vartheta^{*}\left(x, \bar{a}^{*}\right)$.

Let

$$
\varphi(x)=\varphi\left(x, \bar{a}^{*}\right)=\vartheta(x, \bar{a}) \wedge(\forall y)\left[\vartheta^{*}\left(y, \bar{a}^{*}\right) \rightarrow x y=y x \text { (both well defined) }\right] .
$$


So $\psi\left(\mathfrak{C}, \bar{a}^{*}\right) \subseteq \varphi\left(\mathfrak{C}, \bar{a}^{*}\right) \subseteq \vartheta^{*}\left(\mathfrak{C}, \bar{a}^{*}\right) \subseteq \vartheta(\mathfrak{C}, \bar{a})$. So $\psi\left(x, \bar{a}^{*}\right) \vdash \varphi(x, \bar{a})$ and $\varphi(x, \bar{a}) \vdash$ $\vartheta^{*}\left(x, \bar{a}^{*}\right)$ hence the formula $\varphi\left(x, \bar{a}^{*}\right)$ belongs to the type $p_{n}(x)$ which is equal to $\operatorname{Av}\left(A_{n}, D\right)$ hence $\varphi\left(x, \bar{a}^{*}\right) \in \operatorname{Av}\left(\bar{a}^{*}, D\right)$ and $\bar{a}^{*} \subseteq A_{n} \subseteq A \cup B^{G} \cup \cup\{c: c$ realizes $\left.\operatorname{Av}\left(A \cup B^{G}, D\right)\right\}$.

We are done as $\varphi\left(\mathfrak{C}, \bar{a}^{*}\right) \cap G$ is a subgroup and is abelian by the definition of $\varphi(x)$.

4.5 Claim. Assume

(a) $G$ is a definable (infinite) group, (or just type-definable)

(b) every element of $G \backslash\left\{e_{G}\right\}$ commutes with only finitely many others

(c) $G$ has infinitely many pairwise non-conjugate members.

$\underline{\text { Then }} T$ is not strongly ${ }^{+}$dependent.

Proof. Assume first $p^{G}=\{\varphi(x)\}$.

Let $x E y:=[x, y$ are conjugates $]$, clearly it is an equivalence relation, and let

$$
\vartheta\left(x_{1}, x_{2}, y\right):=\left(x_{1}=x_{2} y x_{2}^{-1}\right)
$$

Note that: if $M \models \vartheta\left(x_{1}, z_{1}, y\right) \wedge \vartheta\left(x_{1}, z_{2}, y\right)$ then $M \models z_{1} y z_{1}^{-1}=z_{2} y z_{2}^{-1}$ hence $M \models\left(z_{2}^{-1} z_{1}\right) y=y\left(z_{2}^{-1} z\right)$ so $z_{2}^{-1} z_{1} \in \operatorname{Cm}_{G}(y)$ so $\left\{z: \vartheta\left(x_{1}, z, y\right)\right\}$ is finite. Trivially $\left\{x_{1}: \vartheta\left(x_{1}, x_{2}, y\right)\right\}$ is finite.

We now get a contradiction by 3.10: $\varphi(x), \vartheta\left(x_{1}, x_{2}, y\right)$ satisfies the demands in $(S t)_{1}$ there, which is impossible if $T$ is strongly ${ }^{+}$dependent; so we are done.

If $p^{G}$ is a type use $(S t)_{3}$ of 3.10 .

4.6 Definition. 1) A place $\mathbf{p}$ is a tuple $(p, B, D, *$, inv $)=\left(p^{\mathbf{p}}, B^{\mathbf{p}}, D^{\mathbf{p}}, *_{\mathbf{p}}\right.$, inv $\left._{\mathbf{p}}\right)=$ $(p[\mathbf{p}], B[\mathbf{p}], D[\mathbf{p}], *[\mathbf{p}], \operatorname{inv}[\mathbf{p}])$ such that:

(a) $B$ is a set $\subseteq \mathfrak{C}, D$ is an ultrafilter on $B, p \subseteq \operatorname{Av}(B, D)$

(b) $*$ is a partial two-place function defined with parameters from $B$; we shall write $a *_{\mathbf{p}} b$ or, when clear from the context, $a * b$ or $a b$

(c) inv is a partial unary function definable from parameters in $B$.

1A) $\mathbf{p}$ is non-trival if for every $A$ the type $\operatorname{Av}(A, D)$ is not algebraic.

2) We say $\mathbf{p}$ is weakly a place in a definable group $G$ or type definable group $G \underline{\text { if }}$ $\mathbf{p}$ is a place, $p^{\mathbf{p}} \vdash p^{G}$, the set $B^{\mathbf{p}}$ includes $\operatorname{Dom}\left(p^{G}\right)$ and the operations agree on $p_{\mathbf{p}}[\mathfrak{C}]$ when the place operations are defined.

$2 \mathrm{~A}$ ) If those operations are the same, we say that $\mathbf{p}$ is strongly a place in $G$. 
3) We say $\mathbf{p}_{1} \leq \mathbf{p}_{2}$ if both are places, $B^{\mathbf{p}_{1}} \subseteq B^{\mathbf{p}_{2}}$ and $p^{\mathbf{p}_{2}} \vdash p^{\mathbf{p}_{1}}$ and the operations are same.

4) $\mathbf{p} \leq_{\text {dir }} \mathbf{q}$ if $\mathbf{p} \leq \mathbf{q}$ and $B^{\mathbf{q}} \subseteq A \Rightarrow \operatorname{Av}\left(A, D^{\mathbf{p}}\right)=\operatorname{Av}\left(A, D^{\mathbf{q}}\right)$.

4.7 Definition. 1) A place $\mathbf{p}$ is $\sigma$-closed when:

(a) $\sigma$ has the form $\sigma\left(\bar{x}_{1} ; \ldots, ; \bar{x}_{n(*)}\right)$, a term in the vocabulary of groups

(b) if $\bar{a}_{\ell} \in{ }^{\left(\ell g\left(\bar{x}_{\ell}\right)\right.} \mathfrak{C}$, for $\ell=1, \ldots, n(*)$ and $B \subseteq A$, then $\sigma\left(\bar{a}_{1}, \ldots, \bar{a}_{n(*)}\right)$ is well defined $^{1}$ and realizes $\operatorname{Av}(A, D)$ provided that

(*) $n \leq n(*) \& \ell<\ell g\left(\bar{a}_{n}\right) \Rightarrow a_{n, \ell}$ realizes $\operatorname{Av}\left(A \cup \bar{a}_{1}{ }^{\wedge} \ldots{ }^{\wedge} \bar{a}_{n-1}, D\right)$.

2) A place $\mathbf{p}$ is $\left(\sigma_{1}=\sigma_{2}\right)$-good or satisfies $\left(\sigma_{1}=\sigma_{2}\right)$ when

(a) $\sigma_{\ell}=\sigma_{\ell}\left(\bar{x}_{1}, \ldots, \bar{x}_{n(*)}\right)$ a term in the vocabulary of groups for $\ell=1,2$ (so e.g. $\left(x_{1} x_{2}\right) x_{3}, x_{1}\left(x_{2} x_{3}\right)$ are considered as different terms)

(b) if $\bar{a}_{\ell} \in\left(\ell g\left(\bar{x}_{n}\right) \mathfrak{C}\right.$ for $\ell \leq n$ then $\sigma_{1}\left(\bar{a}_{1} ; \ldots ; \bar{a}_{n(*)}\right)=\sigma_{2}\left(\bar{a}_{1} ; \ldots ; \bar{a}_{n(*)}\right)$ whenever $(*)$ of part (1) holds for $A=B$; so both are well defined.

3) We can replace $\sigma$ in part (1) by a set of terms. Similarly in part (2) for a set of pairs.

4) We may write $x_{\ell}$ instead of $\left\langle x_{\ell}\right\rangle$. So if we write $\sigma\left(\bar{x}_{1} ; \bar{x}_{2}\right)=\sigma\left(x_{1} ; x_{2}\right)=x_{1} x_{2}$ or $\sigma=x_{1} x_{2}$ we mean $x_{1}=x_{1,0}, x_{2}=x_{2,0}, \bar{x}_{1}=\left\langle x_{1,0}\right\rangle, \bar{x}_{2}=\left\langle x_{2,0}\right\rangle$. We may use also $\sigma(\bar{x} ; \bar{y})$ instead of $\sigma\left(\bar{x}_{1} ; \bar{x}_{2}\right)$ and $\sigma(\bar{x} ; \bar{y} ; \bar{z})$ similarly.

4.8 Definition. 1) We say a place $\mathbf{p}$ is a poor semi-group if it is $\sigma$-closed for $\sigma=x y$ and satisfies $\left(x_{1} x_{2}\right) x_{3}=x_{1}\left(x_{2} x_{3}\right)$.

2) We say a place $\mathbf{p}$ is a poor group if it is a poor semi-group and is $\sigma$-closed for $\sigma=\left(x_{1}\right)^{-1} x_{2}$.

3) We say a place $\mathbf{p}$ is a quasi semi-group if for any semi group term $\sigma_{*}(\bar{x}), \mathbf{p}$ is $\sigma$-closed for $\sigma(\bar{x} ; y)=\sigma_{*}(\bar{x}) y$.

4) We say a place $\mathbf{p}$ is a quasi group if for any semi-group terms $\sigma_{1}(\bar{x}), \sigma_{2}(\bar{x})$ we place $\mathbf{p}$ is $\sigma$-closed for $\sigma(\bar{x} ; y)=\sigma_{1}(\bar{x}) y \sigma_{2}(\bar{x})$.

5) We say $\mathbf{p}$ is abelian (or is commutative) if it is ( $x y)$-closed and satisfies $x y=y x$.

6) We say $\mathbf{p}$ is affine if $\mathbf{p}$ is $\left(x y^{-1} z\right)$-closed.

7) We say that a place $\mathbf{p}$ is a pseudo semi-group when: if the terms $\sigma_{1}\left(x_{1}, \ldots, x_{n}\right), \sigma_{2}\left(x_{1}, \ldots, x_{n}\right)$ are equal in semi-groups then $\mathbf{p}$ satisfies $\sigma_{1}\left(x_{1}, \ldots, x_{n}\right)=\sigma_{2}\left(x_{1}, \ldots, x_{n}\right)$.

8) We say that a place $\mathbf{p}$ is a pseudo group if any term $\sigma_{1}\left(x_{1}, \ldots, x_{n}\right), \sigma_{2}\left(x_{1}, \ldots, x_{n}\right)$ which are equal in groups, $\mathbf{p}$ satisfies $\sigma_{1}\left(x_{1}, \ldots, x_{n}\right)=\sigma_{2}\left(x_{1}, \ldots, x_{n}\right)$.

\footnotetext{
${ }^{1}$ so all the stages in the computation of $\sigma\left(\bar{a}_{0} ; \ldots ; \bar{a}_{n(*)}\right)$ should be well defined
} 
4.9 Definition. We say a place $\mathbf{p}$ is a group if $G=G^{\mathbf{p}}=\left(\operatorname{Av}\left(B^{\mathbf{p}}, D\right),{ }^{*} \mathbf{p}, \operatorname{inv}_{\mathbf{p}}\right)$ is a group.

4.10 Claim. 1) The obvious implications hold.

2) If we use $\overline{\mathbf{b}}$ every $\overline{\mathbf{b}}^{\prime}$ realizing the same type has the same properties.

3) For a place $\mathbf{p}$ the assertion "p satisfies $\sigma\left(\bar{x}_{1}, \ldots, \bar{x}_{n(*)}\right)=\sigma\left(\bar{x}_{1}, \ldots, \bar{x}_{n(*)}\right)$ " means just that in Definition 4.7 the term $\sigma\left(a_{1}, \ldots, a_{n}\right)$ is well defined.

We now note that there are places

4.11 Claim. 1) Assume that $G$ is a definable group and $a_{n} \in p^{G}[\mathfrak{C}]$ for $n<\omega$. We define $a_{[u]} \in p^{G}[\mathfrak{C}]$ for any finite non-empty $u \subseteq \omega$ by induction on $|u|$, if $u=\{n\}$ then $a_{[u]}=a_{n}$, if $|u|>1, \max (u)=n$ then $a_{[u]}=a_{[u \backslash\{n\}]} *^{G} a_{n}$ and we are assuming they are all well defined and $a_{\left[u_{1}\right]} \neq a_{\left[u_{2}\right]}$ when $u_{1} \triangleleft u_{2}$. Then we can find $D^{*}, \mathbf{q}$ such that:

(a) $\mathbf{q}$ is a place inside $G$

(b) $\mathbf{q}$ is a poor semi-group and non-trivial

(c) $B^{\mathbf{q}}=B^{G} \cup \bigcup\left\{a_{[u]}: u \subseteq \omega\right.$ is finite $\}$

(d) $D^{*}$ is an ultrafilter on $[\omega]<\aleph_{0}$ such that $(\forall n)\left([\omega \backslash n]<\aleph_{0} \in D^{*}\right)$ and for every $Y \in D^{*}$ we can find $Y^{\prime} \subseteq Y$ from $D^{*}$ closed under convex union, i.e., if $u, v \in Y^{\prime}$ and $\max (u)<\min (v)$ then $u \cup v \in Y^{\prime}$

(e) $D^{\mathbf{q}}=\left\{\left\{a_{[u]}: u \in Y\right\}: Y \in D^{*}\right\}$

$(f)$ if the $a_{n}$ 's commute (i.e. $a_{n} a_{m}=a_{m} a_{n}$ for $n \neq m$ ) then $\mathbf{q}$ is abelian.

Proof. By a well known theorem of Glazer ${ }^{2}$, relative of Hindman theorem saying $D^{*}$ as in clause (d) exists, see Comfort [Cmf77].

4.12 Remark. 1) This can be combined naturally with $\S 1$.

4.13 Claim. 1) Assume

(a) $\mathbf{p}$ is a place in a type-definable group (or much less)

(b) the place $\mathbf{p}$ is a semi-group

(c) $\mathbf{p}$ is commutative (in the sense of Definition $4.7+4.8$, so satisfies $\sigma_{1}(x ; y)=$ $[x * y=y * x]$

(d) if $A \supseteq B^{\mathbf{p}}$ then for some $b, c$ realizing $\operatorname{Av}\left(D^{\mathbf{p}}, A\right), c *_{G} b, b *_{G} c$ are (necessarily well defined, and) distinct.

\footnotetext{
${ }^{2}$ his proof uses the operations from clause (d) of 4.16 and 4.17 below
} 
Then $T$ has the independence property.

2) We can weaken clause (a) to

$(a)^{\prime} \mathbf{p}$ is a place such that for $n<\omega$ and $\left\langle a_{1} a_{1}^{\prime}\right\rangle, \ldots,\left\langle a_{n} a_{n}^{\prime}\right\rangle$ are as in Definition 4.7 and $a_{\ell} \neq a_{\ell}^{\prime} \Leftrightarrow \ell=m$ then $a_{1}, \ldots, a_{m-1}, a_{m} a_{m+1} \ldots a_{n} \neq$ $a_{1}, \ldots, a_{m-1} a_{m}^{\prime} a_{m+1} \ldots a_{m}$.

Remark. This is related to the well known theorems on stable theories (see Zilber and Hrushovski's works).

Proof. 1) We choose $A_{i}, b_{i}, c_{i}$ by induction on $i<\omega$.

In stage $i$ first let $A_{i}=B^{\mathbf{P}} \cup\left\{b_{j}, c_{j}: j<i\right\}$ and add $B^{G}$ if $B^{G} \nsubseteq B^{\mathbf{P}}$. Second, choose $b_{i}, c_{i}$ realizing $\operatorname{Av}\left(A_{i}, D^{\mathbf{P}}\right)$ such that $b_{i} * c_{i} \neq c_{i} * b_{i}$.

Now if $i<j<\omega$ any $a^{\prime} \in\left\{b_{i}, c_{i}\right\}, a^{\prime \prime} \in\left\{b_{j}, c_{j}\right\}$ then $a^{\prime}$ realizes $\operatorname{Av}\left(A_{i}, D^{\mathbf{P}}\right)$ and $a^{\prime \prime}$ realizes $\operatorname{Av}\left(A_{j}, D^{\mathbf{p}}\right)$ which include $\operatorname{Av}\left(A_{i} \cup\left\{a^{\prime}\right\}, D^{\mathbf{p}}\right)$. So by assumption (c), the elements $a^{\prime}, a^{\prime \prime}$ commute in $G$.

So as is well known, for $n<\omega, i_{0}<i_{1}<\ldots<i_{n}$ the element $b_{i_{0}} * b_{i_{1}} * \ldots * b_{i_{n-1}}$ commute in $G$ with $a_{j}$ iff $j \notin\left\{i_{0}, \ldots, i_{n-1}\right\}$ hence $T$ has the independence property. 2) Similarly.

Note that 4.14 is interesting for $G$ with a finite bound on the order of elements as if $a \in G$ has infinite order then $\operatorname{Cm}_{G}\left(\operatorname{Gm}_{G}(a)\right)$ is as desired.

\subsection{Conclusion. [ $T$ is dependent].}

Assume $G$ is a definable group.

1) If $\mathbf{p}$ is a commutative semi-group in $G$, non-trivial, then for some formula $\varphi(x, \bar{a})$ such that $\varphi(\bar{x}) \vdash$ " $x \in G$ " and $\varphi(x, \bar{a}) \in \operatorname{Av}\left(\bar{a}, D^{\mathbf{p}}\right)$ and $G \uparrow \varphi(\mathfrak{C})$ is a commutative place.

2) If $G$ has an infinite abelian subgroup, then it has an infinite definable commutative subgroup.

Proof. 1) By 4.13 for some $A \supseteq B^{\mathbf{P}}$ for every $b, c$ realizing $q:=\operatorname{Av}\left(A, D^{\mathbf{p}}\right)$ we have: the elements of $q(\mathfrak{C})$, which are all in $G$, pairwise commute. By compactness there is a formula $\varphi_{1}(x) \in p[\mathbf{p}]$ such that the elements of $\varphi_{1}(\mathfrak{C}) \cap G$ pairwise commute and without loss of generality $\varphi_{1}(x) \vdash[x \in G]$; note however that this set is not necessarily a subgroup. Let $\varphi_{2}(x):=[x \in G] \wedge(\forall y)\left(\varphi_{1}(y) \rightarrow x * y=y * x\right]$. Clearly $\varphi_{1}(\mathfrak{C}) \subseteq \varphi_{2}(\mathfrak{C}) \subseteq G$ and every member of $\varphi_{2}(\mathfrak{C})$ commutes with every member of $\varphi_{1}(\mathfrak{C})$. So $\varphi(z):=[z \in G] \wedge(\forall y)\left[\varphi_{2}(y) \rightarrow y z=z y\right]$ is first order and defines the center of $G\left\lceil\varphi_{2}[\mathfrak{C}]\right.$ which includes $\varphi_{1}(\mathfrak{C})$, so we are done. 
2) Let $G^{\prime} \subseteq G$ be infinite abelian. Choose by induction on $n<\omega, a_{n} \in G^{\prime}$ as required in 4.13 and then apply it.

4.15 Remark. So 4.14 tells us that having some commutativity implies having alot. If in 4.13 every $a_{[u]}$ is not in any "small" definable set defined with parameters in $B^{\mathbf{P}} \cup\left\{a_{n}: n<\max (u)\right\}$, then also $\varphi(x, \bar{a})$ is not small where small means some reasonably definable ideal.

\subsection{Definition. Assume}

(a) $G$ is a type definable semi-group

(b) $M \supseteq B^{G}$ is $\left(|T|+\left|B^{G}\right|\right)^{+}$-saturated

(c) $\mathfrak{D}$ is the set of ultrafilters $D$ on $M$ such that $p^{G} \subseteq \operatorname{Av}(M, D)$

(d) on $\mathfrak{D}=\mathfrak{D}_{G, M}$ we define an operation

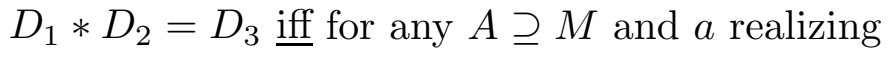

$\operatorname{Av}\left(A, D_{1}\right)$ and $b$ realizing $\operatorname{Av}\left(A+a, D_{2}\right)$ the element $a * b$ realizes $\operatorname{Av}\left(A, D_{3}\right)$.

(e) $I D_{G, M}=\left\{D \in \mathfrak{D}_{G, M}: D * D=D\right\}$

(f) $H_{G, M}^{\text {left }}=\{a \in G$ : for every $D \in \mathfrak{D}$, and $A \supseteq M$ if $b$ realizes $\operatorname{Av}(A+a, D)$ then $a * b$ realizes $\operatorname{Av}(A, D)\}$

(g) $H_{G, M}^{\text {right }}$ similarly using $b * a$

(h) $H_{G, M}=H_{G, M}^{\text {left }} \cap H_{G, M}^{\text {right }}$.

The following as in 4.11.

4.17 Fact: $\mathfrak{D}$ is a semi-group, i.e., associativity holds and the operation is continuous in the second variable hence there is an idempotent (even every non-empty subset closed under $*$ and topologically closed has an idempotent).

Note

4.18 Fact: 1) If $G$ is a group, then

(a) $H_{G, M}^{\text {left }}$ is a subgroup of $G$, with bounded index, and is of the form $\cup\{q(\mathfrak{C})$ : $\left.q \in \mathbf{S}_{G, M}^{\text {left }}\right\}$ for some $\mathbf{S}_{G, M}^{\text {left }} \subseteq \mathbf{S}(M)$

(b) Similarly $H_{G, M}^{\mathrm{right}}, H_{G, M}=H_{G, M}^{\mathrm{right}} \cap H_{G, M}^{\mathrm{left}}$ with $\mathbf{S}_{G, M}^{\mathrm{right}}, \mathbf{S}_{G, M}$. 
2) If $D \in \mathfrak{D}$ is non principal and $\operatorname{Av}(M, D) \in \mathscr{S}_{G, M}^{\text {right }}$, then for any $A \supseteq M$ and element $a$ realizing $\operatorname{Av}(A, D)$ and $b$ realizing $\operatorname{Av}(A+a, D)$ we have

( $\alpha) a *_{G} b$ realizes $\operatorname{Av}(A, D)$

( $\beta)$ also $a^{-1} * b \in D$.

3) $\mathbf{S}_{G, M}^{\text {left }} \subseteq I D_{G, M}$.

4) Similarly for $\mathbf{S}_{G, M}^{\text {left }}, b *_{G} a$.

5) If $D \in \mathfrak{D}, p=\operatorname{Av}(M, D) \in \mathbf{S}_{G, M} \underline{\text { then }}$

(a) $\mathbf{p}=(M, D, *$, inv $)$ is a quasi group

(b) $\left\{a^{-1} b: a, b \in p(M)\right\}$ is a subgroup of $G$ with bound index, in fact is $\left\{a \in \mathfrak{C}: \operatorname{tp}(a, M) \in \mathscr{S}_{G, M}\right\}$. 


\section{$\S 5$ NON-FORKING}

5.1 Hypothesis. $T$ is dependent.

5.2 Definition. [Sh:93] 1) An $\alpha$-type $p=p(\bar{x})$ divides over $B$ if some sequence $\overline{\mathbf{b}}$ and formula $\varphi(\bar{x}, \bar{y})$ witness it which means

(a) $\overline{\mathbf{b}}=\left\langle\bar{b}_{n}: n<\omega\right\rangle$ is an indiscernible sequence over $B$

(b) $\varphi(\bar{x}, \bar{y})$ is a formula with $\ell g(\bar{y})=\ell g\left(\bar{b}_{n}\right)$

(c) $p \vdash \varphi\left(\bar{x}, \bar{b}_{0}\right)$

(d) $\left\{\varphi\left(\bar{x}, \bar{b}_{n}\right): n<\omega\right\}$ is contradictory.

1A) Above we say $\varphi\left(\bar{x}, \bar{b}_{0}\right)$ explicitly divide over $B$.

1B) An $\alpha$-type $p=p(\bar{x})$ splits strongly over $B \underline{\text { when }}$ for some sequence $\overline{\mathbf{b}}$ and formula $\varphi(\bar{x}, \bar{y})$ witness it which means:

$(a),(b)$ as above

(c) $\varphi\left(\bar{x}, \bar{b}_{0}\right), \neg \varphi\left(\bar{x}, \bar{b}_{1}\right) \in p$.

2) An $\alpha$-type $p$ forks over $B$ if for some $\left\langle\varphi_{\ell}\left(\bar{x}, \bar{a}_{\ell}\right): \ell<k\right\rangle$ we have $p \vdash \bigvee_{\ell<k} \varphi_{\ell}\left(\bar{x}, \bar{a}_{\ell}\right)$ and $\left\{\varphi_{\ell}\left(\bar{x}, \bar{a}_{\ell}\right)\right\}$ divides over $B$ for each $\ell<k$ (note: though $\bar{x}$ may be infinite, the formulas are finitary).

We say $p(\bar{x})$ exactly forks (or ex-forks) over $B \underline{\text { when }}$ some $\varphi(\bar{x}, \bar{b}) \in p$ does exactly fork over $B$, which means that for some $\left\langle\varphi_{\ell}(\bar{x}, \bar{b}): \ell<k\right\rangle$ we have: $\varphi(\bar{x}, \bar{b}) \vdash$ $\bigvee_{\ell<k} \varphi_{\ell}(\bar{x}, \bar{b})$ and each $\varphi_{\ell}(\bar{x}, \bar{b})$ explicitly divides over $B$; so we are not allowed to add dummy parameters.

3) We say $C / A$ does not fork over $B$ if letting $\overline{\mathbf{c}}$ list $C, \operatorname{tp}(\overline{\mathbf{c}}, A)$ does not fork over $B$, or what is equivalent $\bar{c} \in{ }^{\omega>} C \Rightarrow \operatorname{tp}(\bar{c}, A)$ does not fork over $B$ (so below we may write claims for $\bar{c}$ and use them for $C$ ).

4) The $m$-type $p$ is f.s. (finitely satisfiable) in $A$ if every finite $q \subseteq p$ is realized by some $\bar{b} \subseteq A$.

5) The $\Delta$-multiplicity of $p$ over $B$ is $\operatorname{Mult}_{\Delta}(p, B)=\sup \left\{\mid\left\{q \uparrow \Delta: p \subseteq q \in \mathbf{S}^{m}(M), q\right.\right.$ does not fork over $B\} \mid: M \supseteq B \cup \operatorname{Dom}(p)\}$.

Omitting $\Delta$ means $\mathbb{L}\left(\tau_{T}\right)$, omitting $B$ we mean $\operatorname{Dom}(p)$. 
5.3 Definition. 1) Let $p=p(\bar{x})$ be an $\alpha$-type and $\Delta$ be a set of $\mathbb{L}\left(\tau_{T}\right)$-formulas of the form $\varphi(\bar{x}, \bar{y})$ and $k \leq \omega$. For a type $p(\bar{x})$ we say that it $(\Delta, k)$-divides over $A$ when some $\overline{\mathbf{b}}, \varphi(\bar{x}, \bar{y})$ witness it which means

(a) $\overline{\mathbf{b}}=\left\langle\bar{b}_{n}: n<2 k+1\right\rangle$ is $\Delta$-indiscernible

(b) $\varphi(\bar{x}, \bar{y}) \in \mathbb{L}\left(\tau_{T}\right)$

(c) $p \vdash \varphi\left(\bar{x}, \bar{b}_{0}\right)$

(d) $\left\{\varphi\left(\bar{x}, \bar{b}_{n}\right): n<2 k+1\right\}$ is $k$-contradictory.

2) For a type $p(\bar{x})$ we say that it $(\Delta, k)$-forks over $B$ when $p \vdash \bigvee_{\ell<n} \varphi_{\ell}\left(x, \bar{a}_{\ell}\right)$ for some $n, \varphi_{\ell}(\bar{x}, \bar{y})$ and $\bar{a}_{\ell}$, where each $\varphi_{\ell}\left(\bar{x}, \bar{a}_{\ell}\right)$ does $(\Delta, k)$-divides over $B$.

5.4 Observation: 0) In Definition 5.2(1), if $p=\{\varphi(x, \bar{b})\}$ then without loss of generality $\bar{b}_{0}=\bar{b}$. If $p$ divides over $B$ then $p$ forks over $B$.

$0 \mathrm{~A})$ Forking is preserved by permuting and repeating the variables. If $\operatorname{tp}\left(\bar{b}^{\wedge} \bar{c}, A\right)$ does not fork over $B$ then so does $\operatorname{tp}(\bar{b}, A)$ and both do not divide over $B$. Similarly for dividing and for exact forking (and later versions).

1) If $p \in \mathbf{S}^{m}(A)$ is finitely satisfiable in $B$, then $p$ does not fork over $B$; hence every type over $M$ does not fork over $M$.

2) If $p \in \mathbf{S}^{m}(A)$ does not fork or just does not divide over $B \subseteq A$, then $p$ does not split strongly over $B$. (Of course, if $p$ divides over $A$ then $p$ forks over $A$ ).

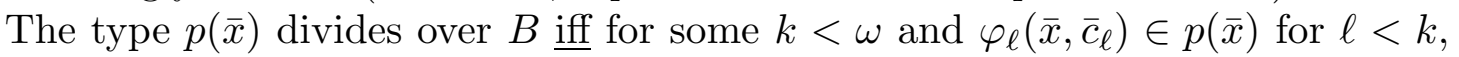
letting $\bar{c}=\bar{c}_{0}{ }^{\wedge} \ldots{ }^{\wedge} \bar{c}_{k-1}$ the formula $\varphi(\bar{x}, \bar{c})=\bigwedge_{\ell<k} \varphi_{\ell}\left(\bar{x}, \bar{c}_{\ell}\right)$ explicitly divides over $B$. Assume the type $\{\varphi(\bar{x}, \bar{b})\}$ or $p(\bar{x}) \in \mathbf{S}^{m}(A)$ for some $A$ or just $p(\bar{x})$ is directed by $\vdash$; i.e. for every finite $q(\bar{x}) \subseteq p(\bar{x})$ there is $\psi(\bar{x}, \bar{b}) \in p(\bar{x})$ such that $\psi(\bar{x}, \bar{x}) \vdash q(\bar{x})$, divides over $B$ iff $\varphi(\bar{x}, \bar{b})$ explicitly divides over $B$, and they imply that in Definition $5.1(1)$, we can choose $\bar{b}_{0}=\bar{b}$ and that $\{\varphi(\bar{x}, \bar{b})\}$ forks over $B$. If $p(\bar{x}) \in \mathbf{S}^{m}(A)$ or just $p(\bar{x})$ is closed under conjunctions (or just is directed by $\vdash$, then $p(\bar{x})$ forks over $B$ iff some $\varphi(\bar{x}, \bar{a}) \in p(\bar{x})$ forks over $B$. The $m$-type $p(\bar{x})$ forks over $B$ iff there is $\varphi(\bar{x}, \bar{a})$ which exactly forks over $B$ such that $p(\bar{x}) \vdash \varphi(\bar{x}, \bar{a})$.

3) [extension property] If an $m$-type $p$ is over $A$ and does not fork over $B$, then some extension $q \in \mathbf{S}(A)$ of $p$ does not fork over $B$.

4) [few non-forking types] For $B \subseteq A$ the set $\left\{p \in \mathbf{S}^{m}(A): p\right.$ does not fork over $B$ (or just does not split strongly) over $B\}$ has cardinality $\leq 2^{2^{|B|+|T|}}$.

If $p(\bar{x})$ does not fork over $M$, then it does not split over $M$.

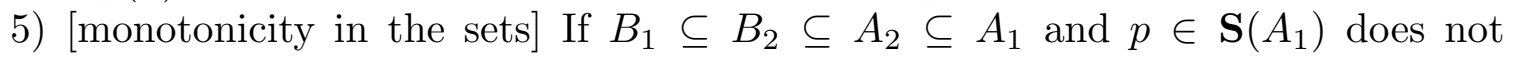
fork/divide over $B_{1}$, then $p\left\lceil A_{2}\right.$ does not fork/divide over $B_{2}$.

6) [indiscernibility preservation] If $\overline{\mathbf{b}}$ is an infinite indiscernible sequence over $A_{1}$ 
and $B \subseteq A_{1} \subseteq A_{2}$ and $\overline{\mathbf{b}} \subseteq A_{2}$ and $\operatorname{tp}\left(\bar{c}, A_{2}\right)$ does not fork over $B$ or just does not divide over $B$ or just does not split strongly over $B \underline{\text { then }} \overline{\mathbf{b}}$ is an (infinite) indiscernible sequence over $A_{1} \cup \bar{c}$.

7) [finite character] If $p$ forks over $B$ iff some finite $q \subseteq p$ does; if $p$ is closed under conjunction (up to equivalence suffices) then we can demand $q=\{\varphi\}$. Similarly for divides ...

8) [monotonicity in the type] If $p(\bar{x}) \subseteq q(\bar{x})$ or just $q(\bar{x}) \vdash p(\bar{x})$ and $p(\bar{x})$ forks over $B$ then $q(\bar{x})$ forks over $B$; similarly for divides; for split strongly this works only for $p(\bar{x}) \subseteq q(\bar{x})$.

9) An $m$-type $p$ is finitely satisfiable in $A$ iff for some ultrafilter $D$ on ${ }^{m} A$ we have $p \subseteq \operatorname{Av}(\operatorname{Dom}(p), D)$.

Remark. 1) Only parts (2), (4), (6) of 5.4 use " $T$ is dependent".

2) If $T$ is unstable then for every $\kappa$ there are some $A$ and $p \in \mathbf{S}(A)$ such that $p$ divides over every $B \subseteq A$ of cardinality $<\kappa$ (use a Dedekind cut with both cofinalities $\geq \kappa)$.

Proof. 0), 0A), 1) Easy.

The proof of part (1) is included in the proof of part (2).

2) Assume toward contradiction that $p$ splits strongly, then for some infinite indiscernible sequence $\left\langle\bar{b}_{n}: n<\omega\right\rangle$ over $B$ and $n<m$ we $^{3}$ have $\left[\varphi\left(\bar{x}, \bar{b}_{n}\right) \equiv \neg \varphi\left(x, \bar{b}_{m}\right)\right] \in$ $p$ (really $p \vdash\left[\varphi\left(\bar{x}, \bar{b}_{n}\right) \equiv \neg \varphi\left(\bar{x}, \bar{b}_{m}\right)\right]$ suffices). By renaming, without loss of generality $n=$ $0, m=1$. Let $\bar{c}_{n}=\bar{b}_{2 n}{ }^{\wedge} \bar{b}_{2 n+1}, \psi\left(\bar{x}, \bar{c}_{n}\right)=\left[\varphi\left(\bar{x}, \bar{b}_{2 n}\right) \equiv \neg \varphi\left(\bar{x}, \bar{b}_{2 n+1}\right)\right]$. Clearly $\left\langle\bar{c}_{n}: n<\omega\right\rangle$ is an indiscernible sequence over $B, p \vdash \psi\left(\bar{x}, \bar{c}_{0}\right)$ and $\left\{\psi\left(\bar{x}, \bar{c}_{n}\right): n<\omega\right\}$ is contradictory as $T$ is dependent.

This proves the first sentence. The second is by the definitions and the third sentence. For the third, the "if" part is obvious, hence let us prove the "only if", so assume that $p(\bar{x})$ divides over $B$, we can find $\varphi\left(\bar{x}, \bar{b}_{0}\right),\left\langle\bar{b}_{n}: n<\omega\right\rangle$ as in Definition $5.2(1)$, i.e. satisfies clauses (a)-(d) there. As $p(\bar{x}) \vdash \varphi\left(\bar{x}, \bar{b}_{0}\right)$, necessarily there is a finite subset $p^{\prime}(\bar{x})$ of $p(\bar{x})$ such that $p^{\prime}(\bar{x}) \vdash \varphi\left(\bar{x}, \bar{b}_{0}\right)$. Let $\left\langle\varphi_{\ell}\left(\bar{x}, \bar{c}_{\ell}\right): \ell<k\right\rangle$ list $p^{\prime}(x)$ and ... Now for each $n<\omega$, the sequences $\bar{b}_{n}, \bar{b}_{0}$ realize the same type over $B$, hence there is a sequence $\bar{c}^{n} \in{ }^{\ell g(\bar{c})} \mathfrak{C}$ such that the sequences $\bar{b}_{0}{ }^{\wedge} \bar{c}, \bar{b}_{n}{ }^{\wedge} \bar{c}^{n}$ realize the same type over $B$ and without loss of generality $\bar{c}^{0}=\bar{c}$. By Ramsey theorem and compactness we can find $\left\langle\bar{d}_{n}: n<\omega\right\rangle$ such that $\bar{b}_{n}{ }^{\wedge} \bar{d}_{n}$ realizes the same type as $\bar{b}_{0}{ }^{\wedge} \bar{c}$ over $B$ and $\left\langle\bar{b}_{n}{ }^{\wedge} \bar{d}_{n}: n<\omega\right\rangle$ is an indiscernible sequence over $B$. So let $F$ be an automorphism of $\mathfrak{C}$ over $B$ which maps $\bar{b}_{0}{ }^{\wedge} \bar{d}_{0}$ to $\bar{b}_{0}{ }^{\wedge} \bar{c}$. So $\left\langle F\left(\bar{d}_{n}\right): n<\omega\right\rangle$ is an indiscernible sequence over $B$ and $F\left(\bar{d}_{0}\right)=\bar{c}$ so $\psi\left(\bar{x}, \bar{d}_{0}\right)=\psi(\bar{x}, \bar{c})=\bigwedge_{\ell<k} \varphi_{\ell}^{\prime}(\bar{x}, \bar{c}) \equiv$

\footnotetext{
${ }^{3}$ recallng $\left[\varphi_{1} \equiv \varphi_{2}\right]$ is the formula $\left(\varphi_{1} \wedge \varphi_{2}\right) \vee\left(\neg \varphi_{1} \wedge \neg \varphi_{2}\right)$
} 
$\bigwedge_{\ell<k} \varphi_{\ell}\left(\bar{x}, \bar{c}_{\ell}\right) \vdash \varphi\left(\bar{x}, \bar{b}_{0}\right)=\varphi\left(\bar{x}, F\left(\bar{b}_{0}\right)\right)$.

Necessarily also $n<\omega \Rightarrow \psi\left(\bar{x}, \bar{d}_{n}\right) \vdash \varphi\left(x, \bar{b}_{n}\right)$ and as $\left\{\varphi\left(\bar{x}, \bar{b}_{n}\right): n<\omega\right\}$ is contradictory, so is $\left\{\psi\left(\bar{x}, \bar{d}_{n}\right): n<\omega\right\}$. So $\left\langle F\left(\bar{d}_{n}\right): n<\omega\right\rangle$ examplifies that $\psi\left(\bar{x}, \bar{d}_{0}\right)=\psi(\bar{x}, \bar{c})$ explicitly divides over $B$ as promised.

The fourth and fifth sentences are obvious.

3) By the definitions (or see [Sh:93]).

4) Easy or see [Sh 3]; e.g. by part (3) without loss of generality $B=M, A=|N|$ is $\|M\|^{+}$-saturated. Now if $\bar{a}_{\ell} \in{ }^{m} N$ realizes the same type over $M$ for $\ell=1,2$ then for some $\bar{c}_{n} \in{ }^{m} N$ for $n=1,2, \ldots,\left\langle\bar{a}_{\ell}\right\rangle^{\wedge}\left\langle\bar{c}_{1}, \bar{c}_{2}, \ldots\right\rangle$ is indiscernible over $M$.

5) Easy.

6) By part (2) and transitivity of "equality of types" and Fact 5.5 below. 7), 8), 9) Easy.

We implicitly use the trivial:

5.5 Fact. 1) If $I$ is a linear order, $\bar{s}_{0}, \bar{s}_{1}$ are increasing $n$-tuples from $I$ then

$\circledast_{\omega}$ there is a linear order $J \supseteq I$ such that for $\ell \in\{0,1\}$ there is an indiscernible sequence $\left\langle\bar{t}_{k}^{\ell}: k<\omega\right\rangle$ of increasing $n$-tuples from $J$ such that $\bar{t}_{k+1}^{0}=\bar{t}_{k+1}$ for $k<\omega$ and $\ell=0,1 \Rightarrow \bar{s}_{\ell}=\bar{t}_{0}^{\ell}$; indiscernible means for quantifier free formulas in the order language, i.e., in the vocabulary $\{<\}$ is satisfaction in $J$. If $I$ has no last element or no first element then we can take $I=J$.

2) Similar for $\left\langle\bar{b}_{t}: t \in I\right\rangle$ an infinite indiscernible sequence over $A$ in $\mathfrak{C}$.

Proof. 1) Let $J \supseteq I$ be with no last element. Choose for $k=1,2, \ldots$ an increasing sequence $\bar{t}_{k}$ of length $n$ from $J$ such that $2 \leq k<\omega \Rightarrow \operatorname{Rang}\left(\bar{s}_{0}{ }^{\wedge} \bar{s}_{1}\right)<\operatorname{Rang}\left(\bar{t}_{k}\right)<$ $\operatorname{Rang}\left(\bar{t}_{k+1}\right)$. So $\left\langle\bar{s}_{\ell}\right\rangle^{\wedge}\left\langle\bar{t}_{1}, \bar{t}_{2}, \ldots\right\rangle$ is an indiscernible sequence in $J$ for $\ell=0,1$.

2) Easy.

5.6 Definition. 1) Let $p$ be an $m$-type satisfying $p \uparrow B_{2} \in \mathbf{S}^{m}\left(B_{2}\right)$. We say that $p$ strictly does not divide over $\left(B_{1}, B_{2}\right)$, (normally $B_{1} \subseteq B_{2}$; when $B_{1}=B_{2}=B$ we may write "over $B$ ") when:

(a) $p$ does not divide over $B_{1}$

(b) if $\left\langle\bar{c}_{n}: n<\omega\right\rangle$ is an indiscernible sequence over $B_{2}$ such that $\bar{c}_{0}$ realizes $p$ and $A$ is any set satisfying $\operatorname{Dom}(p) \cup B_{1} \subseteq A$, then there is an indiscernible sequence $\left\langle\bar{c}_{n}^{\prime}: n<\omega\right\rangle$ over $A$ such that $\bar{c}_{0}^{\prime}$ realizes $p$ and $\operatorname{tp}\left(\left\langle\bar{c}_{n}: n<\right.\right.$ $\left.\omega\rangle, B_{2}\right)=\operatorname{tp}\left(\left\langle\bar{c}_{n}^{\prime}: n<\omega\right\rangle, B_{2}\right)$. 
1A) "Strictly divide" is the negation.

2) We say that $p$ strictly forks over $\left(B_{1}, B_{2}\right)$ iff $p \vdash \bigvee_{\ell<n} \varphi_{\ell}$ for some $\left\langle\varphi_{\ell}: \ell<n\right\rangle$ such that $\left(p\left\lceil B_{2}\right) \cup\left\{\varphi_{\ell}\right\}\right.$ strictly divides over $\left(B_{1}, B_{2}\right)$ for each $\ell<n$.

3) An $m$-type $p(\bar{x})$ strictly does not fork over $\left(B_{1}, B_{2}\right)$ when: $p(\bar{x})$ does not fork over $B_{1}$, so $p(\bar{x}) \uparrow B_{2} \in \mathbf{S}^{m}\left(B_{2}\right)$ and if $\left\langle\bar{c}_{n}: n<\omega\right\rangle$ is an indiscernible sequence over $B_{2}$ of sequences realizing $p(\bar{x})$ and $C \supseteq B_{1} \cup \operatorname{Dom}(p(\bar{x}))$ and $q(\bar{x}) \in \mathbf{S}^{m}(C)$ extend $p(\bar{x})$ and does not fork over $B_{1}$ then there is an indiscernible sequence $\left\langle\bar{c}_{n}^{\prime}: n<\omega\right\rangle$ over $C$ realizing $\operatorname{tp}\left(\left\langle\bar{c}_{n}: n<\omega\right\rangle, B_{2}\right)$ such that $\bar{c}_{0}^{\prime}$ realizes $q(\bar{x})$; note that "strictly does not fork" is not defined as "does not strictly forks"; to stress we may write "strictly* does not fork".

We shall need some statements concerning "strictly* does not fork" parallel to those on "does not fork".

5.7 Observation. 0) In clause (b) of Definition 5.6(1) we can weaken the assumption " $\bar{c}_{0}$ realizes $p$ " to " $\bar{c}_{0}$ realizes $p \uparrow B_{2}$ ".

1) "Strictly does not divide/fork over $\left(B_{1}, B_{2}\right)$ " is perserved by permuting the variables, repeating variables and by automorphisms of $\mathfrak{C}$ and if it holds for $\operatorname{tp}\left(\bar{b}^{\wedge} \bar{c}, A\right)$ so $B_{2} \subseteq A$ then it holds for $\operatorname{tp}(\bar{b}, A)$. Similarly for "does not strictly fork".

1A) The $m$-type $p(\bar{x})$ strictly does not divide over $\left(B_{1}, B_{2}\right)$ iff $p(\bar{x}) \uparrow B_{2} \in \mathbf{S}^{m}\left(B_{2}\right)$ and $\left(p(\bar{x})\left\lceil B_{2}\right) \cup q(\bar{x})\right.$ strictly does not divide over $\left(B_{1}, B_{2}\right)$ for every finite $q(\bar{x}) \subseteq p(\bar{x})$.

2 ) If $p$ strictly* does not fork over $\left(B_{1}, B_{2}\right)$ then $p$ does not strictly fork over $\left(B_{1}, B_{2}\right)$ which implies that $p$ strictly does not divide over $\left(B_{1}, B_{2}\right)$.

3 ) If $p$ strictly does not divide over $\left(B_{1}, B_{2}\right)$ then $p$ does not divide over $B_{1}$.

4) If $p$ does not strictly* fork over $B$ then $p$ does not fork over $B$.

$5)$ If $p$ is an $m$-type which strictly* does not fork over $\left(B_{1}, B_{2}\right)$ and $B_{1} \cup \operatorname{Dom}(p) \subseteq A$ then there is $q \in \mathbf{S}^{m}(A)$ extending $p$ which strictly* does not fork over $\left(B_{1}, B_{2}\right)$. Similarly does not strictly fork. If $p_{1}(\bar{x}) \subseteq p_{2}(\bar{x})$ and $p_{1}(\bar{x})$ strictly does not fork over $\left(B_{1}, B_{2}\right)$ and $p_{2}(\bar{x})$ does not fork over $B_{1}$ then $p_{2}(\bar{x})$ strictly does not fork over $\left(B_{1}, B_{2}\right)$.

6) If $B_{1} \subseteq B_{1}^{\prime} \subseteq B_{2}^{\prime}=B_{2}$ and $p(\bar{x}) \vdash p^{\prime}(\bar{x})$ and $p(\bar{x})$ does not strictly divide/fork over $\left(B_{1}, B_{2}\right)$ and $p^{\prime} \uparrow B_{2}^{\prime}$ is complete, i.e. $\in \mathbf{S}^{m}\left(B_{2}^{\prime}\right)$ then $p^{\prime}(\bar{x})$ does not strictly divide/fork over $\left(B_{1}^{\prime}, B_{2}^{\prime}\right)$.

7) In Definition 5.6(1), clause (b) the case $A=\operatorname{Dom}(p) \cup B_{2}$ suffice.

8) If $p$ strictly forks over $\left(B_{1}, B_{2}\right)$ then for some finite $q \subseteq p$ the type $q \cup\left(p\left\lceil B_{2}\right)\right.$ strictly forks over $\left(B_{1}, B_{2}\right)$. Moreover, for some finite $B_{2}^{\prime} \subseteq B_{2},(p$ is an $m$-type), if $B_{1} \cup B_{2}^{\prime} \subseteq B_{2}^{\prime \prime}, p^{\prime}$ is an $m$-type extending $q$ and $p^{\prime} \uparrow B_{2} \in \mathbf{S}^{m}\left(B_{2}\right)$ then $p^{\prime}$ strictly forks over $\left(B_{1}, B_{2}^{\prime \prime}\right)$. Similarly for strictly divide.

9) If $M \subseteq A, p=\operatorname{tp}(\bar{b}, A)$ and $\operatorname{tp}(A, M+\bar{b})$ is finitely satisfiable in $M$, then $p$ 
strictly does not fork over $M$.

Proof. Easy, e.g.,

$0)$ The new version is stronger hence it implies the one from the definition.

So assume that $p$ is an $m$-type, $p \uparrow B_{2} \in \mathbf{S}^{m}\left(B_{2}\right)$ and $p$ strictly does not divide over $\left(B_{1}, B_{2}\right)$ and we shall prove the new version of clause (b). I.e., we have $\left\langle\bar{c}_{n}: n\langle\omega\rangle\right.$ is an indiscernible sequence over $B_{2}$ and $\bar{c}_{0}$ realizes $p \nmid B_{2}$. Let $\bar{c}_{0}^{\prime \prime} \in{ }^{m} \mathfrak{C}$ realizes $p$ hence it realizes $p \uparrow B_{2}$, but $p \uparrow B_{2} \in \mathbf{S}^{m}\left(B_{2}\right)$ so $\operatorname{tp}\left(\bar{c}_{0}, B_{2}\right)=\operatorname{tp}\left(\bar{c}_{0}^{\prime \prime}, B_{2}\right)$. We can deduce that there is an automorphism $F$ of $\mathfrak{C}$ over $B_{2}$ which maps $\bar{c}_{0}$ to $\bar{c}_{0}^{\prime \prime}$, and define $\bar{c}_{n}^{\prime \prime}=F\left(\bar{c}_{n}\right)$.

Now $\left\langle F\left(\bar{c}_{n}\right): n<\omega\right\rangle$ satisfies the assumption of clause (b) from Definition 5.6(1) hence there is an indiscernible sequence $\left\langle\bar{c}_{n}^{\prime}: n<\omega\right\rangle$ over $A$ such that $\operatorname{tp}\left(\left\langle\bar{c}_{n}^{\prime}: n<\right.\right.$ $\left.\omega\rangle, B_{2}\right)=\operatorname{tp}\left(\left\langle F\left(\bar{c}_{n}^{\prime}\right): n<\omega\right\rangle, B_{2}\right)$, but the latter is equal to $\operatorname{tp}\left(\left\langle c_{n}: n<\omega\right\rangle, B_{2}\right)$ so we are done.

1A) Let $p^{\prime}(\bar{x}):=p(\bar{x})\left\lceil B_{2}\right.$ so $p^{\prime}(\bar{x}) \in \mathbf{S}^{m}\left(B_{2}\right)$ and let $A=\operatorname{Dom}(p) \cup B_{1}$. Now by parts $(0),(1)$ and the definitions

$\bigotimes_{1} p(\bar{x})$ strictly does not divide above $B_{2} \underline{\text { iff }}$ the following holds

(a) $p(\bar{x})$ does not divide over $B_{1}$

(b) if $\overline{\mathbf{c}}=\left\langle\bar{c}_{n}: n\langle\omega\rangle\right.$ is an indiscernible sequence over $B_{2}$ then (where $\ell g\left(\bar{x}_{n}\right)=m$ ) the set $\cup\left\{p\left(\bar{x}_{n}\right): n<\omega\right\} \cup \Gamma_{\overline{\mathbf{c}}}^{1} \cup \Gamma_{\overline{\mathbf{c}}}^{2}$ is finitely satisfiable where:

$$
\begin{array}{ll}
(\alpha) \quad \Gamma_{\overline{\mathbf{c}}}^{1}=\left\{\varphi\left(\bar{x}_{0}, \ldots, \bar{x}_{n-1}, \bar{b}\right): n<\omega, \varphi\left(\bar{x}_{0}, \ldots, \bar{x}_{n-1}, \bar{y}\right) \in \mathbb{L}\left(\tau_{1}\right)\right. \\
\left.\bar{b} \subseteq B_{2} \text { and } \models \varphi\left[\bar{c}_{0}, \ldots, \bar{c}_{n-1}, \bar{b}\right]\right\} \\
(\beta) \quad \Gamma_{\mathbf{c}}^{2}=\left\{\varphi\left(\bar{x}_{k_{0}}, \ldots, \bar{x}_{k_{n-1}}, \bar{a}\right) \equiv \varphi\left(\bar{x}_{\ell_{0}}, \ldots, \bar{x}_{\ell_{n-1}}, \bar{a}\right): n<\omega\right. \text { and } \\
& k_{0}<\ldots<k_{n-1} \text { and } \ell_{0}<\ldots<\ell_{n-1} \text { and } \bar{a} \subseteq A \text { and } \\
& \left.\varphi\left(\bar{x}_{0}, \ldots, \bar{x}_{n-1}, \bar{y}\right) \in \mathbb{L}\left(\tau_{T}\right)\right\} .
\end{array}
$$

But the demand in $\otimes$ has finite character.

$2)$ For the first implication assume $p(\bar{x})$ strictly* does not fork over $\left(B_{1}, B_{2}\right)$ but $p(\bar{x})$ strictly forks over $\left(B_{1}, B_{2}\right)$ so $p(\bar{x}) \vdash \bigvee_{\ell<n} \varphi_{\ell}\left(\bar{x}, \bar{a}_{i}\right)$ and $\left(p(\bar{x}) \uparrow B_{2}\right) \cup\left\{\varphi_{\ell}(\bar{x}, \bar{a})\right.$ strictly does not divide over $\left(B_{1}, B_{2}\right)$ hence by part $(1 \mathrm{~A})$ the type $p_{\ell}(\bar{x}):=p(\bar{x}) \cup\left\{\varphi_{\ell}\left(\bar{x}, \bar{a}_{\ell}\right)\right\}$ strictly divides over $\left(B_{1}, B_{2}\right)$ for each $\ell<m$.

But by Definition 5.6(3) the type $p(\bar{x})$ does not fork over $B_{1}$ hence we can choose $\ell<n$ such that $p_{\ell}(\bar{x})$ does not fork over $B_{1}$. As $p_{\ell}$ strictly divides over $\left(B_{1}, B_{2}\right)$ in Definition 5.6(1), clause (a) or clause (b) there fail, for $p_{\ell}(\bar{x})$, but clause (a) holds by the choice of $\ell$, hence clause (b) fails and let $\left\langle\bar{c}_{n}: n\langle\omega\rangle\right.$ and $A$ exemplifies it; so without loss of generality $A \supseteq \bar{a}_{\ell} \cup B_{1} \cup B_{2} \cup \operatorname{Dom}(p)$. Now let 
$q(\bar{x}) \in \mathbf{S}^{m}(A)$ extends $p_{\ell}(\bar{x})$ and does not fork over $B_{1}$ and apply Definition 5.6(3) with $p(\bar{x}), A, q(\bar{x}),\left\langle\bar{c}_{n}: n<\omega\right\rangle$ here standing for $p(\bar{x}), C, q(\bar{x}),\left\langle\bar{c}_{n}: n<\omega\right\rangle$ there; so we can find $\left\langle\bar{c}_{n}^{\prime}: n<\omega\right\rangle$ indiscernible over $A$ realizing $\operatorname{tp}\left(\left\langle\bar{c}_{n}: n<\omega\right\rangle, B_{2}\right)$ such that $\bar{c}_{0}^{\prime}$ realizing $q(\bar{x})$. This contradicts the choice of $A,\left\langle\bar{c}_{n}: n<\omega\right\rangle$. The second implication holds by the earlier parts.

5) The third sentence holds by the definition. The first sentence follows by the third and 5.4(3). The second sentence follows by the definition.

6) Without loss of generality $\operatorname{Dom}(p) \cup B_{2}^{\prime} \subseteq A$ and it suffices to prove the case of "strictly does not divide". Recall that by part (1) in Claim 5.4(A), clause (b) we can demand only " $\bar{c}_{0}$ realizes $p \uparrow B_{2}$ " and for any such $\left\langle\bar{c}_{n}: n<\omega\right\rangle$ choose is $\bar{c}_{0}^{\prime \prime}$ realizing $p$ hence $\bar{c}_{0}$ and $\bar{c}_{0}^{\prime \prime}$ realizes the same type over $B_{2}$ hence there is automorphism $F$ of $\mathfrak{C}$ over $B_{2}$ mapping $\bar{c}_{0}$ to $\bar{c}_{0}^{\prime \prime}$ and use the definition for $\left\langle F\left(\bar{c}_{n}\right): n<\omega\right\rangle$.

7) By Ramsey theorem and compactness.

9) Use an ultrafilter $D$.

The next claim is a parallel of: every type over $A$ does not fork over some "small" $B \subseteq A$. If we have " $p$ is over $A$ implies $p$ does not fork over $A$ " we could have improvement.

More elaborately, note that if $M$ is a dense linear order and $p \in \mathbf{S}(M)$, then $p$ actually corresponds to a Dedekind cut of $M$. So though in general $p$ is not definable, $p \nmid\{c \in M: c \notin(a, b)\}$ is definable whenever $(a, b)$ is an interval of $M$ which includes the cut. So $p$ is definable in large pieces. The following (as well as 5.20) realizes the hope that something in this direction holds for every dependent theory.

5.8 Claim. If $p \in \mathbf{S}^{m}(A)$ and $B \subseteq A$, then we can find $C \subseteq A$ of cardinality $\leq|T|$ such that:

$\circledast$ if $\left\langle\bar{a}_{n}: n\langle\omega\rangle\right.$ is an indiscernible sequence over $B \cup C$ such that $\bar{a}_{0} \subseteq A$ and $\operatorname{tp}\left(\bar{a}_{0}, B \cup C\right)$ strictly* does not fork over $B$ and $\left\{\varphi\left(\bar{x}, \bar{a}_{n}\right): n<\omega\right\}$ is contradictory or just $\varphi\left(\bar{x}, \bar{a}_{0}\right)$ exactly forks over $B \cup C$, then $\neg \varphi\left(x, \bar{a}_{0}\right) \in p$.

Remark. The first case in $\circledast$, (the one on " $\left\{\varphi\left(\bar{x}, \bar{a}_{n}\right): n<\omega\right\}$ is contradictory, says, in other words no formula in $p$ divides over $B \cup C$ when the type of the sequence of parameters over $B \cup C$ does not fork over $B$.

5.9 Conclusion. 1) For every $p \in \mathbf{S}^{m}(A)$ and $B \subseteq A$, we can find $C \subseteq A,|C| \leq|T|$ such that: 
$\circledast$ if $\left\langle\bar{a}_{n}: n<\omega\right\rangle$ is an indiscernible sequence over $B \cup C$ satisfying $\bar{a}_{0} \cup \bar{a}_{1} \subseteq A$ and $\operatorname{tp}\left(\bar{a}_{0} \wedge \bar{a}_{1}, B \cup C\right)$ strictly* does not fork over $B$, then for any $\varphi$

(*) $\varphi\left(x, \bar{a}_{0}\right) \in p$ iff $\varphi\left(x, \bar{a}_{1}\right) \in p$.

2) For every $\bar{x}=\left\langle x_{\ell}: \ell<m\right\rangle$ and formula $\varphi=\varphi(\bar{x} ; \bar{y})$ for some finite $\Delta \subseteq \mathbb{L}(T)$ we have:

if $p \in \mathbf{S}^{m}(A), B \subseteq A$, then for some finite $C \subseteq A$ (in fact $|C|<$ $f(m, \varphi, T)$ for some function $f)$, we have:

if $\left\langle\bar{a}_{\ell}: \ell\langle k\rangle\right.$ is $\Delta$-indiscernible sequence over $B \cup C$ and $\operatorname{tp}_{\Delta}\left(\bar{a}_{0}{ }^{\wedge} \bar{a}_{1}, B \cup\right.$

$C)$ does not fork over $A$, then $\varphi\left(x, \bar{a}_{0}\right) \in p \Leftrightarrow \varphi\left(x, \bar{a}_{1}\right) \in p$.

3) The local version of 5.8 holds with a priori finite bound on $C$.

Proof of 5.8. By induction on $\alpha<|T|^{+}$we try to choose $C_{\alpha}, \bar{a}_{\alpha}, k_{\alpha}$ and $\left\langle\bar{a}_{\alpha, n}^{k}: n<\right.$ $\omega\rangle$ and $\varphi_{\alpha}\left(\bar{x}, \bar{y}_{\alpha}\right), \varphi_{\alpha, k}\left(\bar{x}, \bar{y}_{\alpha, k}\right)$ such that:

$\bigotimes(a) \quad C_{\alpha}=\cup\left\{\bar{a}_{\beta}: \beta<\alpha\right\} \cup B$

(b) $\left\langle\bar{a}_{\alpha, n}^{k}: n<\omega\right\rangle$ is an indiscernible sequence over $C_{\alpha}$ for $k<k_{\alpha}$

(c) $\bar{a}_{\alpha} \subseteq A$ and $\bar{a}_{\alpha}=\bar{a}_{\alpha, 0}^{k}$ for $k<k_{\alpha}$

(d) $\varphi_{\alpha}\left(\bar{x}, \bar{a}_{\alpha}\right) \in p$

(e) $\left\{\varphi_{\alpha, k}\left(\bar{x}, \bar{a}_{\alpha, n}^{k}\right): n<\omega\right\}$ is contradictory

(f) $\operatorname{tp}\left(\bar{a}_{\alpha}, B \cup C_{\alpha}\right)$ strictly* does not fork over $B$

(g) $\varphi_{\alpha}\left(\bar{x}, \bar{a}_{\alpha}\right) \vdash \bigvee_{k<k_{\alpha}} \varphi_{\alpha, k}\left(\bar{x}, \bar{a}_{\alpha, 0}^{k}\right)$.

If for some $\alpha<|T|^{+}$we are stuck, $C=C_{\alpha} \backslash B$ is as required. So assume that we have carried the induction and we shall eventually get a contradiction.

By induction on $\alpha<|T|^{+}$we choose $D_{\alpha}, F_{\alpha}, \bar{b}_{\beta},\left\langle\bar{b}_{\beta, n}^{k}: n<\omega\right\rangle$ for $\beta<\alpha$ such that (but $\bar{b}_{\alpha, n}^{k}$ are defined in the $(\alpha+1)$-th stage):

$\boxplus(\alpha) \quad F_{\alpha}$ is an elementary mapping, increasing continuous with $\alpha$

( $\beta) \operatorname{Dom}\left(F_{\alpha}\right)=C_{\alpha}$ and $\operatorname{Rang}\left(F_{\alpha}\right) \subseteq D_{\alpha}$

$(\gamma) \quad D_{\alpha}=\operatorname{Rang}\left(F_{\alpha}\right) \cup \bigcup\left\{\bar{b}_{\beta, n}^{k}: \beta<\alpha, k<k_{\alpha}\right.$ and $\left.n<\omega\right\}$ so $D_{\alpha} \subseteq \mathfrak{C}$ is increasing continuous

$(\delta)\left\langle\bar{b}_{\alpha, n}^{k}: n<\omega\right\rangle$ is an indiscernible sequence over $D_{\alpha} \supseteq F_{\alpha}\left(C_{\alpha}\right)$ and $\bar{b}_{\alpha, 0}^{k}=\bar{b}_{\alpha}$ 
(ع) $\quad F_{\alpha+1}\left(\bar{a}_{\alpha}\right)=\bar{b}_{\alpha}$ and $\operatorname{tp}\left(\bar{b}_{\alpha}, D_{\alpha}\right)$ does not fork over $F_{\alpha}(B)$

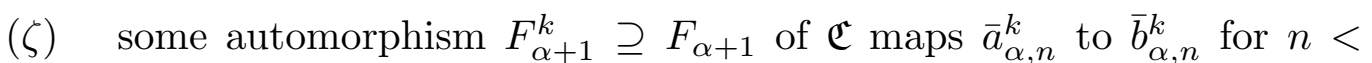
$\omega, k<k_{\alpha}$.

For $\alpha=0, \alpha$ limit this is trivial. For $\alpha=\beta+1$, clearly $F_{\alpha}\left(\operatorname{tp}\left(\bar{a}_{\alpha}, C_{\alpha}\right)\right)$ is a type in $\mathbf{S}^{<\omega}\left(F_{\alpha}\left(C_{\alpha}\right)\right)$ which strictly* does not fork over $F_{\alpha}(B)=F_{0}(B)$ hence by $5.4(3)$ has an extension $q_{\alpha} \in \mathbf{S}^{<\omega}\left(D_{\alpha}\right)$ which does not fork over $F_{0}(B)$ and let $\bar{b}_{\alpha}$ realize it. Let $F_{\alpha+1} \supseteq F_{\alpha}+1$ be the elementary mapping extending $F_{\alpha}$ with domain $C_{\alpha+1}$ mapping $\bar{a}_{\alpha}$ to $\bar{b}_{\alpha}$. Let $F_{\alpha+1}^{k} \supseteq F_{\alpha}$ be an automorphism of $\mathfrak{C}$ as required by clauses $(\delta)+(\zeta) ; F_{\alpha+1}^{k}$ exists as $\operatorname{tp}\left(\bar{a}_{\alpha}, B \cup C_{\alpha}\right)$ strictly* does not fork over $B$ and let $\bar{b}_{\alpha, n}^{k}=F_{\alpha+1}^{k}\left(\bar{a}_{\alpha, n}^{k}\right)$ for $n<\omega, k<k_{\alpha}$. So $D_{\alpha+1}$ and $F_{\alpha+1}$ are well defined.

Having carried the induction let $F \supseteq \bigcup\left\{F_{\alpha}: \alpha<|T|^{+}\right\}$be an automorphism of $\mathfrak{C}$. We claim that for each $\alpha<|T|^{+}$and $k<k_{\alpha}$, for every $\beta \in\left[\alpha,|T|^{+}\right]$we have

$(*)_{\alpha, \beta}\left\langle\bar{b}_{\alpha, n}^{k}: n<\omega\right\rangle$ is an indiscernible sequence over $D_{\alpha} \cup \bigcup\left\{\bar{b}_{\gamma}: \gamma \in[\alpha+1, \beta)\right\}$.

We prove this by induction on $\beta$. For $\beta=\alpha$ this holds by clause $(\delta)$, for $\beta \equiv \alpha+1$ this is the same as for $\beta=\alpha$. For $\beta$ limit use the definition of indiscernibility. For $\beta=\zeta+1>\alpha+1$ use $\operatorname{tp}\left(\bar{b}_{\zeta}, D_{\zeta}\right)$ does not fork over $F_{0}(B)$ hence over $D_{\alpha} \cup\left\{F_{\gamma+1}\left(\bar{b}_{\gamma}\right)\right.$ : $\alpha<\gamma<\zeta\}$ by 5.4(5); so by the induction hypothesis and 5.4(6) clearly $(*)_{\alpha, \beta}$ holds.

From $\alpha<\beta \leq|T|^{+} \Rightarrow(*)_{\alpha, \beta}$ we can conclude

$(* *)$ for any $n<\omega$ and $\alpha_{0}<\ldots<\alpha_{n-1}<|T|^{+}$and $\nu \in \prod_{\ell<n} k_{\alpha_{\ell}}$ and $\eta \in{ }^{n} 2$ the sequences $\bar{b}_{\alpha_{0}, 0}^{\nu(0)} \bar{b}_{\alpha_{1}, 0}^{\nu(1) \wedge} \ldots{ }^{\wedge} \bar{b}_{\alpha_{n-1}, 0}^{\nu(n-1)}$ and $\bar{b}_{\alpha_{0}, \eta(0)}^{\nu(0)} \wedge^{\wedge} \bar{b}_{\alpha_{1}, \eta(1)}^{\nu(1)} \wedge^{\wedge}{ }^{\wedge} \bar{b}_{\alpha_{n-1}, \eta(n-1)}^{\nu(n-1)}$ realize the same type over $B$.

[Why? By induction on $\ell(*)=\max \{\ell: \eta(\ell)=1$ or $\ell=-1\}$. If $\ell(*)=-1$ then the two sequences are the same so $(* *)$ holds trivially. Let $\rho \in{ }^{n_{2}} 2$ be defined by $\rho(\ell)$ is 0 if $\ell \neq \ell(*)$ and $\eta(\ell)$ otherwise, so the induction hypothesis apply to $\rho$ so it suffices to prove that $\bar{b}_{\eta}^{\nu}, \bar{b}_{\rho}^{\nu}$ realizes the same type over $B$. Now assume $\ell(*) \in\{0, \ldots, n-1\}$ and use $(*)_{\alpha_{\ell(*)},|T|^{+}}$for

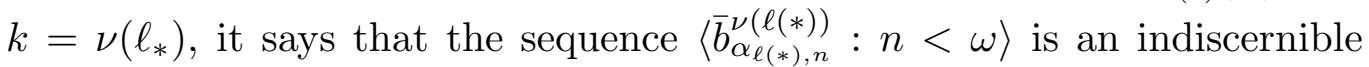
sequence over $D_{\alpha_{\ell(*)}} \cup\left\{\bar{b}_{\gamma}: \gamma \in\left[\alpha_{\ell(*)+1},|T|^{+}\right)\right\}$.

The second part in the union includes

$$
\begin{aligned}
& \left\{\bar{b}_{\alpha_{\ell(*)+1}, 0}^{\nu(\ell(*)-1)}, \ldots, \bar{b}_{\alpha_{n-1}, 0}^{\nu(n-1)}\right\}=\left\{\bar{b}_{\alpha_{\ell(*)+1}, \eta(\ell(*)+1)}^{\nu(\ell)}, \ldots, \bar{b}_{\alpha_{n-1}, \eta(n-1)}^{\nu(n-1)}\right\} \\
& =\left\{\bar{b}_{\alpha_{\ell(*)+1}^{\nu}}^{\nu(\ell(*))}, \ell(*)+1, \ldots, \bar{b}_{\alpha_{n-1}, \rho(n-1)}^{\nu(n-1)}\right\}
\end{aligned}
$$


by the choice of $\ell(*)$, and the first part of the union includes the rest so it suffices to have that $\bar{b}_{\alpha_{\ell(*)}}=\bar{b}_{\alpha_{\ell(*)}, 0}^{\nu(\ell(*))}=b_{\alpha_{\ell(*)}, \rho(\ell(*))}^{\nu(\ell(*)}$ and $\pm \bar{b}_{\alpha_{\ell(*)}, \eta(\ell(*))}^{\nu(\ell(*))}=$ $b_{\alpha_{\ell(*)}, 1}^{\nu(\ell())}$ realizes the same type $D_{\alpha_{\ell(*)}} \cup\left\{\bar{b}_{\gamma}: \gamma=\alpha_{\ell(*)+1}, \ldots, \alpha_{n-1}\right.$, which holds.]

Let $\bar{c}$ realize $F(p)$. For each $\alpha<|T|^{+}, \varphi_{\alpha}\left(x, \bar{a}_{\alpha}\right) \in p$ hence $\varphi_{\alpha}\left(x, \bar{b}_{\alpha}\right) \in F(p)$. Also $\varphi_{\alpha}\left(x, \bar{a}_{\alpha}\right) \vdash \bigvee_{k<k_{\alpha}} \varphi_{\alpha, k}\left(x, a_{\alpha, 0}^{k}\right)$ hence by clause $(\zeta)$ we have $\varphi_{\alpha}\left(x, \bar{b}_{\alpha}\right) \vdash \bigvee_{k<k_{\alpha}} \varphi_{\alpha, k}\left(x, a_{\alpha, 0}^{k}\right)$ hence we can choose $k(\alpha)<k_{\alpha}$ such that $\mathfrak{C} \models \varphi\left[\bar{c}, \bar{a}_{\alpha, 0}^{k(\alpha)}\right]$.

Now as $\left\{\varphi_{\alpha, k(\alpha)}\left(x, \bar{b}_{\alpha, n}^{k(\alpha)}\right): n<\omega\right\}$ is contradictory there is $n=n[\alpha]<\omega$ such that $\mathfrak{C} \models \neg \varphi_{\alpha, k(\alpha)}\left(\bar{c}, \bar{b}_{\alpha, n}^{k(\alpha)}\right)$ whereas $\mathfrak{C} \models \varphi_{\alpha, k(\alpha)}\left[\bar{c}, \bar{b}_{\alpha, 0}^{k(\alpha)}\right]$; by renaming without loss of generality $\mathfrak{C} \models \neg \varphi_{\alpha, k(\alpha)}\left[c, \bar{b}_{\alpha, n}^{k(\alpha)}\right]$ for $\alpha<|T|^{+}, n \in[1, \omega)$. Now if $n<\omega, \alpha_{0}<$ $\ldots<\alpha_{n-1}<|T|^{+}$and $\eta \in{ }^{n} 2$ then $\mathfrak{C} \models \bigwedge_{\ell<m} \varphi_{\alpha_{\ell}, k\left(\alpha_{\ell}\right)}\left(\bar{c}, \bar{b}_{\alpha_{\ell}, \eta(\ell)}^{k\left(\alpha_{\ell}\right)}\right)^{\text {if }(\eta(\ell)=0)}$ hence $\mathfrak{C} \models(\exists \bar{x})\left[\bigwedge_{\ell<n} \varphi_{\alpha_{\ell}, k\left(\alpha_{\ell}\right)}\left(\bar{x}, \bar{b}_{\alpha_{\ell}, \eta(\ell)}\right)^{\mathrm{if}(\eta(\ell)=0)}\right]$ hence by $(* *)$ we have $\mathfrak{C} \models(\exists \bar{x})\left[\bigwedge_{\ell<n} \varphi_{\alpha_{\ell}, k\left(\alpha_{\ell}\right)}\left(\bar{x}, \bar{b}_{\alpha_{\ell}, 0}^{k\left(\alpha_{\ell}\right)}\right)^{\mathrm{if}(\eta(\ell)=0)}\right]$.

Hence the independence property holds, contradiction. 5.8

Proof of 5.9. 1) Follows from 5.8 by 5.4(2).

2) By 5.8 and compactness or repeating the proof.

3) Similarly.

5.10 Claim. 1) Assume $p$ is a type, $B \subseteq M, \operatorname{Dom}(p) \subseteq M$ and $M$ is $|B|^{+}$saturated. Then

(A) $p$ does not fork over $B$ iff $p$ has a complete extension over $M$ which does not fork over $B$ iff $p$ has a complete extension over $M$ which does not divide over $B$ iff $p$ has a complete extension over $M$ which does not split strongly over $B$

$(B)$ if $p=\operatorname{tp}(\bar{c}, M)$ and $\varphi(\bar{x}, \bar{a}) \in p$ forks over $B$, then for some $\left\langle\bar{a}_{n}: n<\omega\right\rangle$ indiscernible over $B,\left\{\bar{a}_{n}: n<\omega\right\} \subseteq M, \bar{a}_{0}=a$ and $\neg \varphi\left(\bar{x}, \bar{a}_{1}\right) \in p$, and of course, $\varphi\left(\bar{x}, \bar{a}_{0}\right) \in p$

$(C)$ if $p \in \mathbf{S}^{m}(M)$ then $p$ forks over $B$ iff $p$ exactly forks over $B$.

2) Assume $\operatorname{tp}\left(C_{1} / A\right)$ does not fork over $B \subseteq A$ and $\operatorname{tp}\left(C_{2},\left(A \cup C_{1}\right)\right)$ does not fork over $B \cup C_{1}$. Then $\left.\operatorname{tp}\left(C_{1} \cup C_{2}\right), A\right)$ does not fork over $B$. 
Proof. 1) Read the definitions.

Clause $(A)$ :

First implies second by 5.4(3), second implies third by Definition 5.2 or 5.4(2), third implies fourth by 5.4(2). If the first fails, then $p \vdash \bigvee_{\ell<k} \varphi_{\ell}\left(\bar{x}, \bar{a}_{\ell}\right)$ for some $k$ where each $\varphi_{\ell}\left(\bar{x}, \bar{a}_{\ell}\right)$ divides over $B$; let $\left\langle\bar{a}_{\ell, n}: n<\omega\right\rangle$ witness this hence by $5.4(2)$ without loss of generality $\bar{a}_{\ell}=\bar{a}_{\ell, 0}$. As $M$ is $|B|^{+}$-saturated, without loss of generality $\bar{a}_{\ell, n} \subseteq$ $M$. So for every $q \in \mathbf{S}^{m}(M)$ extending $p$, for some $\ell<k, \varphi_{\ell}\left(\bar{x}, \bar{a}_{\ell}\right) \in q$ but for every large enough $n, \neg \varphi_{\ell}\left(\bar{x}, \bar{a}_{\ell, n}\right) \in q$, so $q$ splits strongly, i.e., fourth fails. So fourth implies first, "closing the circle".

Clause $(B)$ :

Similar.

2) Let $M$ be $|B|^{+}$-saturated model such that $A \subseteq M$. By 5.4(3) there is an elementary mapping $f_{1}$ such that $f_{1} \uparrow A=\operatorname{id}_{A}$ and $\operatorname{Dom}\left(f_{1}\right)=C_{1} \cup A$ and $f_{1}\left(C_{1}\right) / M$ does not fork over $B$. Similarly we can find an elementary mapping $f \supseteq f_{1}$ such that $\operatorname{Dom}(f)=C_{1} \cup C_{2} \cup A$ and $f\left(C_{2}\right) /\left(M \cup f\left(C_{1}\right)\right)$ does not fork over $A \cup f\left(C_{1}\right)$. By $5.4(2), f_{1}\left(C_{1}\right) / M$ does not split strongly over $B$. Again by $5.4(2)$, $f\left(C_{2}\right) /\left(M \cup f_{1}\left(C_{1}\right)\right)$ does not split strongly over $B \cup f_{1}\left(C_{1}\right)$. Together they imply that if $\overline{\mathbf{b}} \subseteq M$ is an infinite indiscernible sequence over $B$ then it is an indiscernible sequence over $f\left(C_{1}\right) \cup B$ and even over $f\left(C_{2}\right) \cup\left(f\left(C_{1}\right) \cup B\right)$ (use the two previous sentences and 5.4(6)). But this means that $f\left(C_{1}\right) \cup f\left(C_{2}\right) / M$ does not split strongly over $B$, (here the exact version of strong splitting we choose is immaterial as $M$ is $|B|^{+}$-saturated). So by $5.10(1)$ we get that $f\left(C_{1}\right) \cup f\left(C_{2}\right) / M$ does not fork over $B$ hence $f\left(C_{1} \cup C_{2}\right) / A$ does not fork over $B$ but $f \supseteq \operatorname{id}_{A}$ so by $5.4(2)$ also $C_{1} \cup C_{2} / A$ does not split strongly over $B$.

5.11 Conclusion. 1) If $M$ is $|B|^{+}$-saturated and $B \subseteq M$ and $p \in \mathbf{S}^{n}(M)$ then $p$ does not fork over $B$ iff $p$ does not strongly split over $B$.

2) If $A=|M|$, then in Conclusion 5.9(1) we can replace strong splitting by dividing.

Proof. 1) By 5.10(1A).

2) By part (1).

5.12 Definition. 1) We say $\left\langle\bar{a}_{t}: t \in J\right\rangle$ is a non-forking sequence over $(B, A)$ when $B \subseteq A$ and for every $t \in J$ the type $\operatorname{tp}\left(\bar{a}_{t}, A \cup \bigcup\left\{\bar{a}_{s}: s<_{J} t\right\}\right)$ does not fork over $B$.

2) We say that $\left\langle\bar{a}_{t}: t \in J\right\rangle$ is a strict non-forking sequence over $\left(B_{1}, B_{2}, A\right)$ if $B_{1} \subseteq B_{2} \subseteq A$ and for every $t \in J$ the type $\operatorname{tp}\left(\bar{a}_{t}, A \cup \bigcup\left\{\bar{a}_{s}: s<_{J} t\right\}\right)$ strictly* does not fork over $\left(B_{1}, B_{2}\right)$, see Definition 5.6(3). If $B_{1}=B_{2}$ we may write $\left(B_{1}, A\right)$ 
instead of $\left(B_{1}, B_{1}, A\right)$.

3) We say $\mathscr{A}=\left(A,\left\langle\left(\bar{a}_{\alpha}, B_{\alpha}\right): \alpha<\alpha^{*}\right\rangle\right)$ is an $\mathbf{F}_{\kappa}^{f}$-construction or $\left\langle\left(\bar{a}_{\alpha}, B_{\alpha}\right): \alpha<\right.$ $\left.\alpha^{*}\right\rangle$ an $\mathbf{F}_{\kappa}^{f}$-construction over $A$ if $B_{\alpha} \subseteq A_{\alpha}:=A \cup \bigcup\left\{\bar{a}_{\beta}: \beta<\alpha\right\}$ has cardinality $<\kappa$ and $\operatorname{tp}\left(\bar{a}_{\alpha}, A_{\alpha}\right)$ does not fork over $B_{\alpha}$.

4) We can above replace $\bar{a}_{t}$ by $A_{t}$ meaning for some/every $\bar{a}_{t}$ listing $A_{t}$ the demand holds.

5.13 Claim. 1) Assume

(a) $\left\langle\bar{a}_{t}: t \in J\right\rangle$ is a strict non-forking sequence over $(B, B, A)$

(b) $\left\langle\bar{b}_{t, n}^{\varepsilon}: n<\omega\right\rangle$ is an indiscernible sequence over $A$, each $\bar{b}_{t, n}$ realizing $\operatorname{tp}\left(\bar{a}_{t}, A\right)$ for each $t \in J, \varepsilon<\zeta_{t}$.

$\underline{\text { Then }}$ we can find $\bar{a}_{t, n}$ for $t \in J, n<\omega, \varepsilon<\zeta_{t}$ such that

$(\alpha)\left\langle\bar{a}_{t, n}: n<\omega\right\rangle$ is an indiscernible sequence over $A_{1} \cup\left\{\bar{a}_{s, n}: n<\omega, s \in\right.$ $J \backslash\{t\}\}$

(ß) $\operatorname{tp}\left(\left\langle\bar{a}_{t, n}: n<\omega\right\rangle, A\right)=\operatorname{tp}\left(\left\langle\bar{b}_{t, n}: n<\omega\right\rangle, A\right)$

$(\gamma) \bar{a}_{t, 0}=\bar{a}_{t}$

Proof. We prove by induction on $|J|$, both parts.

Case 1: $J$ is finite.

We prove this by induction on $n=|J|$, for $n=0,1$ this is trivial; assume we have proved for $n$ and we shall prove for $n+1$. Let $\lambda=(|A|+|T|)^{+}$.

So let $J=\left\{t_{\ell}: \ell \leq n\right\}$ with $t_{\ell}$ increasing with $\ell$. First we can find an indiscernible sequence $\left\langle\bar{c}_{t_{0}, \alpha}^{\varepsilon}: \alpha<\lambda\right\rangle$ over $A$ such that $\bar{c}_{t_{0}, 0}^{\varepsilon}=\bar{a}_{t_{0}}$ and for some automorphism $F_{\varepsilon}$ of $\mathfrak{C}$ over $B$ we have $k<\omega \Rightarrow F\left(\bar{b}_{t_{0}, k}^{\varepsilon}\right)=\bar{c}_{t_{0}, k}^{\varepsilon}$ and let $A^{\prime}:=A \cup\left\{\bar{c}_{t_{0}, \alpha}^{\varepsilon}: \alpha<\lambda\right\}$. [This is possible by Definition 5.6.]

Second, we can choose $\bar{a}_{t_{\ell}}^{\prime}$ by induction on $\ell$ such that $\bar{a}_{t_{0}}^{\prime}=\bar{a}_{t_{0}}$ and if $\ell>0$ then $\operatorname{tp}\left(\bar{a}_{t_{\ell}}^{\prime}, A^{\prime} \cup \bigcup\left\{\bar{a}_{t_{m}}^{\prime}: m=1, \ldots, \ell-1\right\}\right)$ strictly* does not fork over $B$ and the two sequences $\bar{a}_{t_{0}}{ }^{\wedge} \ldots{ }^{\wedge} \bar{a}_{t_{\ell}}, \bar{a}_{t_{0}}^{\prime}{ }^{\wedge} \ldots{ }^{\wedge} \bar{a}_{t_{\ell}}^{\prime}$ realizes the same type over $A$. We can do it by $5.7(5)$ and "strictly* does not fork" being preserved by elementary mapping. By 5.10(2) the type $\left.\operatorname{tp}\left(\bar{a}_{t_{1}}^{\prime}{ }^{\wedge} \ldots{ }^{\wedge} \bar{a}_{t_{n}}^{\prime}, A^{\prime}\right\}\right)$ does not fork over $B$ hence by $5.4(6)$ the sequence $\left\langle\bar{a}_{t_{0}, \alpha}: \alpha<\lambda\right\rangle$ is an indiscernible sequence over $A \cup\left(\bar{a}_{t_{1}}^{\prime}{ }^{\wedge} \ldots{ }^{\wedge} \bar{a}_{t_{n}}^{\prime}\right)$.

As $\operatorname{tp}\left(\bar{a}_{t_{\ell}}, A \cup\left\{\bar{a}_{t_{m}}: m<i\right\}\right)$ strictly does not fork over $(B, A)$ without loss of generality $\left\langle\bar{b}_{t_{\ell}, m}: m<\omega\right\rangle$ is an indiscernible sequence over $A^{\prime}$ such that each member realizes $\operatorname{tp}\left(\bar{a}_{t_{\ell}}, A^{\prime}\right)$.

Now we use the induction hypothesis with $B, A^{\prime},\left\langle\bar{a}_{t_{\ell}}^{\prime}: \ell=1, \ldots, n\right\rangle,\left\langle\bar{b}_{t_{\ell}, m}: m<\right.$ $\omega\rangle$ for $\ell=1, \ldots, n$ and let $\left\langle\bar{a}_{t_{\ell}, n}^{\prime}: n<\omega\right\rangle$ for $\ell=1, \ldots, n$ be as in the claim. 
By [Sh 715] for some $\alpha^{*}<\lambda$ the sequence $\left\langle\bar{c}_{t_{0}, \alpha}^{\varepsilon}: \alpha \in\left[\alpha^{*}, \alpha^{*}+\omega\right)\right\rangle$ is an indiscernible sequence over $A \cup \bigcup\left\{\bar{a}_{t_{\ell}, m}^{\prime}: m<\omega, \ell=1, \ldots, n\right\}$ and as $A^{\prime}=A \cup$ $\left\{\bar{a}_{t_{0}, \alpha}^{\prime}: \alpha<\lambda\right\}$ clearly for $\ell=1, \ldots, n$ the sequence $\left\langle\bar{a}_{t_{\ell}, m}^{\prime}: m<\omega\right\rangle$ is indiscernible over $A \cup \bigcup\left\{\bar{a}_{t_{k}, m}^{\prime}: k \in\{1, \ldots, n\} \backslash\{\ell\}\right.$ and $\left.m<\omega\right\} \cup \bigcup\left\{\bar{a}_{\alpha^{*}+m}^{\prime}: m<\omega\right\}$. But we know that $\left\langle\bar{a}_{t_{0}, \alpha}^{\prime}: \alpha<\alpha^{*}+\omega\right\rangle$ is an indiscernible sequence over $A \cup\left\{\bar{a}_{t_{\ell}}^{\prime}\right.$ : $\ell=1, \ldots, n\}$, hence the sequence $\bar{a}_{t_{\alpha}, \alpha^{*}}^{\prime} \bar{a}_{t_{1}}^{\prime}{ }^{\wedge}{ }^{\wedge} \bar{a}_{t_{n}}^{\prime}$ realizes over $A$ the same type as $\bar{a}_{t_{0}, 0}^{\prime} \bar{a}_{t_{1}}^{\prime}{ }^{\wedge} \ldots{ }^{\wedge} \bar{a}_{t_{n}}^{\prime}$ hence realizes over $A$ the same type as $\bar{a}_{t_{0}}{ }^{\wedge} \bar{a}_{t_{1}}{ }^{\wedge} \ldots{ }^{\wedge} \bar{a}_{t_{n}}$. So for some automorphism $F$ of $\mathfrak{C}, F\left\lceil A=\mathrm{id}_{A}, \ell=1, \ldots, n \Rightarrow \bar{a}_{t_{\ell}}=F\left(\bar{a}_{t_{\ell}, 0}^{\prime}\right)\right.$ and $\bar{a}_{t_{0}}=F\left(\bar{a}_{t_{0}, \alpha^{*}}^{\prime}\right)$ and let $\bar{a}_{t_{\ell}, m}=F\left(\bar{a}_{t_{\ell}, m}^{\prime}\right)$ for $\ell=1, \ldots, n$ and $m<\omega$ and $\bar{a}_{t_{0}, m}=F\left(\bar{a}_{t_{0}, \alpha^{*}+m}^{\prime}\right)$.

So we are done.

Case 2: $J$ infinite.

By Case $1+$ compactness.

Remark. Can we use just no dividing?

5.14 Claim. 1) Assume $\left\langle A_{t}: t \in J\right\rangle$ is a non-forking sequence over $(B, A)$ and $C_{t} \subseteq \mathfrak{C}$ for $t \in J$. Then we can find $\left\langle f_{t}: t \in J\right\rangle$ such that

(a) $f_{t}$ is an elementary mapping with domain

$$
A \cup A_{t} \cup C_{t}
$$

(b) $f_{t} \uparrow\left(A \cup A_{t}\right)$ is the identity

(c) $\operatorname{tp}\left(A_{t}, A \cup \bigcup\left\{A_{s} \cup f_{s}\left(C_{s}\right): s<t\right\}\right.$ does not fork over $B$.

2) If in addition $\operatorname{tp}\left(C_{t}, A \cup A_{t}\right)$ does not fork over $A \cup A_{t}$ then we can add

$(c)^{+}\left\langle A_{t} \cup f_{t}\left(C_{t}\right): t \in J\right\rangle$ is a non-forking sequence over $(B, A)$.

5.15 Remark. 1) We may consider $\mathbf{F}^{f}$-construction, i.e., $\mathscr{A}=\left(A,\left\langle a_{\alpha}^{B_{i}}: \alpha<\alpha^{*}\right\rangle\right)$ is an $\mathbf{F}^{f}$-construction, when

(a) $B_{i} \subseteq A_{i}:=A \cup\left\{a_{j}: j<i\right\}$

(b) $\operatorname{tp}\left(a_{i}, A_{i}\right)$ does not fork over $B_{i}$

(c) $\left|B_{i}\right|<\kappa$. 
1A) We may replace above $\alpha$ by a linear order $I$, not necessarily well founded. 2) In 5.14(2) we may weaken the assumption to: for every $A^{\prime} \supseteq A, A_{t} \cup C_{t} / A$ can be embedded to a complete non-forking type over $A^{\prime}$.

Proof. 1) As in the proof of 5.8.

2) Similarly.

5.16 Claim. 1) Assume

(a) $\left\langle A_{t}: t \in J\right\rangle$ is a non-forking sequence over $(B, A)$.

Then for any initial segment $I$ of $J, \operatorname{tp}\left(\bigcup\left\{A_{t}: t \in J \backslash I\right\}, A \cup \bigcup\left\{A_{t}: t \in I\right\}\right)$ does not fork over $B$.

2) Assume (a) and

(b) $\left\langle\bar{a}_{t, n}: n<\omega\right\rangle$ is an indiscernible sequence over $A$,

(c) $\bar{a}_{t, n} \in \omega>\left(A_{t}\right)$

(d) $\left\langle\bar{a}_{t, n}: n<\omega\right\rangle$ is an indiscernible sequence over $A \cup \bigcup\left\{A_{s}: s<_{J} t\right\}$.

$\underline{\text { Then }}\left\langle\left\langle\bar{a}_{t, n}: n<\omega\right\rangle: t \in J\right\rangle$ are mutually indiscernible over A. Also for any non-zero $k<\omega$ and $t_{0}<\ldots<t_{k-1}$ in $J$ the sequences $\left\langle\bar{a}_{t_{\ell}, n}: n<\omega\right\rangle$ for all $\ell<k$ are mutually indiscernible over $A \cup \bigcup\left\{A_{s}: \neg\left(t_{0} \leq s \leq t_{k-1}\right)\right\}$.

5.17 Question: If $n_{\ell}<\omega$ for $\ell<n$ do the sequences $\left\langle\bar{a}_{t_{0}, n_{0}}{ }^{\wedge} \bar{a}_{t_{1}, n_{1}}{ }^{\wedge} \ldots{ }^{\wedge} \bar{a}_{t_{k-1}, n_{k-1}}\right\rangle$ and $\left\langle\bar{a}_{t_{0}, 0} \hat{a}_{t_{1}, 0}{ }^{\wedge} \ldots{ }^{\wedge} \bar{a}_{t_{k-1}, 0}\right\rangle$ realize the same type over $A \cup \bigcup\left\{A_{s}: s<_{J} t_{0}\right.$ or $\left.s_{J}>t_{k-1}\right\}$. Need less?

Remark. A statement similar to 5.16(1) for $\mathbf{F}_{\kappa}^{f}$-construction holds.

Proof. 1) If $J \backslash I$ is finite, we prove this by induction on $|J \backslash I|$ using $5.10(2)$. The general case follows by $5.4(7)$.

2) It is enough to prove the second sentence. For $k=1$ this follows by $5.4(6)$ $+5.10(2)$ using part (1) with $A \cup \bigcup\left\{A_{s}: s<t\right\},\left\langle A_{r}: r \in J, r>t\right\rangle$ instead $A,\left\langle A_{r}: r \in J\right\rangle$.

For $k+1>1$, let us be given $t_{0}<_{J} \ldots<_{J} t_{k}$. Use the case $k=1$ for each $t_{\ell}$ and combine.

5.18 Remark. 1) Recall that by 5.10 if $p \in \mathbf{S}^{m}(M), M$ is quite saturated, then dividing is the same as forking for the type $p$. 
5.19 Claim. Assume that for every set $B$, if $p(\bar{x}) \in \mathbf{S}^{m}(B)$ then $p$ does not fork over $B$.

Assume that $\left\langle\bar{a}_{t}: t \in J\right\rangle$ is a non-forking sequence over $(B, A)$ and $A=|M|$.

1) For every (finite sequence) $\bar{b}$ the set $\left\{t: \bar{b} /\left(A \cup \bar{a}_{t}\right)\right.$ forks over $\bigcup_{s<t} \bar{a}_{s} \cup A$ has cardinality $\leq|T|$.

2) For each $\varphi(\bar{x}, \bar{y}, \bar{z})$ and $k<\omega$ for some $n=n_{\varphi(\bar{x}, \bar{y}), k}$ the set $W_{\bar{b}}^{\varphi}:=\{t$ : $\operatorname{tp}_{\varphi}\left(\bar{b}, A \cup \bar{a}_{t}\right)$ has a subset with $\leq k$ members which forks over $\left.\bigcup_{s<t} \bar{a}_{s} \cup A\right\}$ has $\leq n$ members.

Proof. 1) By (2).

2) Fix $k$. Assume toward contradiction that this fails for $n$. We can find $t_{0}<_{I}$ $t_{1}<_{J} \ldots<_{J} t_{n-1}$ from $W_{\bar{b}}^{\varphi}$.

Now for every $u \subseteq\{0, \ldots, n-1\}$ there is $\bar{b}_{u}$ realizing $\operatorname{tp}\left(\bar{b}, A \cup\left\{\bar{a}_{t_{\ell}}: \ell \in u\right\}\right)$ such that $\operatorname{tp}\left(\bar{b}_{u}, A \cup\left\{\bar{a}_{t_{\ell}}: \ell<n\right\}\right)$ does not fork over $A \cup\left\{\bar{b}_{t_{\ell}}: \ell \in u\right\}$. Now for each $\ell<n$ we can find $q_{\ell} \subseteq \operatorname{tp}_{\varphi}\left(\bar{b}, A \cup \bar{a}_{t_{\ell}}\right)$ and with $\leq k$ members which forks over $\bigcup_{s<t} \bar{a}_{s} \cup A$; let $A_{\ell}=A \cup \bar{a}_{t_{0}} \cup \ldots \cup \bar{a}_{t_{\ell}-1}$. Clearly $\ell \in u \Rightarrow q_{\ell} \subseteq \operatorname{tp}\left(\bar{b}_{u}, A \cup\left\{\bar{a}_{t_{m}}: m<n\right\}\right)$. Now if $\ell \in n \backslash u$, let $i_{\ell, 0}<\ldots<i_{\ell, m(\ell)-1}<n$ list $u \backslash \ell$ so $\operatorname{tp}\left(\bar{a}_{i_{\ell, m}}, A_{\ell} \cup \bar{a}_{t_{\ell}} \cup \bar{a}_{t_{i_{\ell, 0}}} \ldots \cup\right.$ $\left.\bar{a}_{t_{i_{\ell, m}(\ell)-1}}\right)$ does not fork over $A$ for $m<m(\ell)$ and $\operatorname{tp}\left(\bar{b}_{u}, A \cup \bar{a}_{t_{\ell}} \cup \bar{a}_{i_{\ell}} \cup \ldots \cup \bar{a}_{t_{i_{\ell, m}(\ell)-1}}\right)$ does not fork over $A \cup\left\{\bar{b}_{t_{k}}: k \in u\right\} \subseteq A_{\ell} \cup\left\{\bar{a}_{t_{i_{\ell, 0}}} \cup \ldots \cup \bar{a}_{t_{i_{\ell, m}(\ell)-1}}\right)$ hence by $5.10(2)$ $+5.4(0)$ the type $\operatorname{tp}\left(\bar{b}_{u}, A_{\ell} \cup \bar{a}_{t_{\ell}}\right)$ does not fork over $A_{\ell}$. Hence now $\operatorname{as~tp}_{\varphi}\left(\bar{b}, A \cup \bar{a}_{t_{\ell}}\right)$ has the subset $q_{\ell}$ with $\leq k$ members which forks over $\bigcup_{s<t} \bar{a}_{s} \cup A$, by monotonicity $q_{\ell}$ it forks also over its subset $A_{\ell}$, hence $q_{\ell}$ forks over $A_{\ell}$ hence by the previous sentence $q_{\ell} \nsubseteq \operatorname{tp}\left(b_{u}, A \cup \bar{b}_{t_{\ell}}\right)$ so $\neg \wedge q_{\ell} \in \operatorname{tp}\left(b_{u}, A \cup \bar{b}_{t_{\ell}}\right)$. As for our fixed $k$ this holds for every $n$, we get that $T$ has the indpendence property contradiction. $\square_{5.19}$

5.20 Claim. Assume that $p(\bar{x})$ is a type of cardinality $<\kappa$ which does not fork over $A$. Then for some $B \subseteq A$ of cardinality $<\kappa+|T|^{+}$, the type $p$ does not fork over $B$.

Proof. Without loss of generality $p$ is closed under conjunction.

For any finite sequence $\bar{\varphi}=\left\langle\left(\varphi_{\ell}\left(\bar{x}, \bar{y}_{\ell}\right), n_{\ell}\right): \ell<n\right\rangle$ and formula $\psi(x, \bar{c}) \in p$ and set $B \subseteq A$ we define 


$$
\begin{aligned}
\Gamma_{B, \bar{\varphi}, \psi(\bar{x}, \bar{c})=\{} & \left.(\forall x)\left(\psi(x, \bar{c}) \rightarrow \bigvee_{\ell<n} \varphi_{\ell}\left(\bar{x}, \bar{y}_{\ell, 0}\right)\right)\right\} \cup \\
& \left\{\neg(\exists \bar{x}) \bigwedge_{n \in w} \varphi_{\ell}\left(\bar{x}, y_{\ell, n}\right): \ell<n \text { and } w \in[\omega]^{n_{\ell}}\right\} \cup \\
& \left\{\vartheta\left(y_{\ell, m_{1}}, \ldots, y_{\ell, m_{k}}, \bar{b}\right)=\vartheta\left(y_{\ell, 0}, \ldots, y_{\ell, k}, \bar{b}\right):\right. \\
& \left.\bar{b} \subseteq B, \vartheta \in \mathbb{L}\left(\tau_{T}\right), m_{1}<\ldots<m_{k}<\omega\right\} .
\end{aligned}
$$

Now as $p$ does not fork over $A$, clearly for any $\bar{\varphi}$ as above and $\psi(\bar{x}, \bar{c}) \in p$ the set $\Gamma_{A, \bar{\varphi}, \psi(\bar{x}, \bar{c})}$ is inconsistent. Hence for some finite set $B=B_{\bar{\varphi}, \psi(x, \bar{c})} \subseteq A$ the set $\Gamma_{B, \bar{\varphi}, \psi(x, \bar{c})}$ is inconsistent. Now $B^{*}=\cup\left\{B_{\bar{\varphi}, \psi(\bar{x}, \bar{c})}: \psi(\bar{x}, \bar{c}) \in p\right.$ and $\bar{\varphi}$ is as above $\}$ is as required.

The following is another substitute for "every type $p$ does not fork over a small subset of $\operatorname{Dom}(p) "$.

5.21 Claim. Assume that for every set $B$, if $p \in \mathbf{S}^{<\omega}(B)$ then $p$ does not fork over $B$.

Assume $p \in \mathbf{S}^{m}(M)$ and $B \subseteq M$. Then we can find $C$ such that

$$
(*)_{1} C \subseteq M \text { and }|C| \leq|T| \text { and }
$$

$(*)_{M, B, C}^{p}$ if $D \subseteq M$ and $\operatorname{tp}(D / B \cup C)$ does not fork over $B$ then $p \uparrow(B \cup D)$ does not fork over $B \cup C$.

Proof. Follows by 5.19.

5.22 Definition. Assume that $C=|M|, M$ is $\kappa$-saturated $A \subseteq M,|A|<\kappa$ and $p \in \mathbf{S}^{m}(M)$ does not split over $A$. For any set $B(\subseteq \mathfrak{C})$ let $p^{[B, \bar{A}]}$ be $q \uparrow B$ where $q \in \mathbf{S}^{m}(M \cup B)$ is the unique type in $\mathbf{S}^{m}(M \cup B)$ which does not split over $A$.

5.23 Observation. 1) In Definition 5.22, $p^{[B, A]}$ is well defined.

2) In 5.22 instead " $C$ is $\kappa^{+}$-saturated; $|A|<\kappa$ " it suffices to assume that every $q \in \mathbf{S}^{<\omega}(B)$ is realized in $C$ that is $C$ is full over $B$.

3) $p^{\left[A, B_{1}\right]} \subseteq p^{\left[A, B_{2}\right]}$ if $B_{1} \subseteq B_{2}$. 
5.24 Claim. 1) If the triple $(A, C, p)$ is as in 5.23(2), $A \subseteq A_{0}$ and $\bar{a}_{n} \in{ }^{m} \mathfrak{C}$ realizes $p^{\left[A_{n}, C\right]}$ for $n<\omega$ where $A_{n}=A_{0} \cup \bigcup\left\{\bar{a}_{\ell}: \ell<n\right\}$ then $\left\langle\bar{a}_{n}: n<\omega\right\rangle$ is an indiscernible sequence over $A_{0}$. Also $\operatorname{tp}\left(\left\langle\bar{a}_{n}: n<\omega\right\rangle, A_{0}\right)$ is determined by $\left(A, C, p, A_{0}\right)$ and as $p$ determines $C$, we call it $p^{\left[A, A_{0}, \omega\right]}$.

Proof. See [Sh:c, II,§1] or [Sh 3].

5.25 Claim. Assume that

(a) $C \supseteq A$ is full over $A$

(b) $p_{0}, p_{1} \in \mathbf{S}^{m}(C)$ does not split over $A$

(c) $p_{0}^{[A, A, \omega]}=p_{1}^{[A, A, \omega]}$.

Then $p_{0}=p_{1}$.

Proof. Let $\left\langle\bar{a}_{n}^{\ell}: n<\omega\right\rangle$ realize $p_{\ell}^{[A, A, \omega]}$ so by clause (c) of the assumption

$(*)_{1} \bar{a}_{0}^{0}, \ldots, \bar{a}_{n-1}^{0}$ and $\bar{a}_{0}^{1}{ }^{\wedge} \ldots{ }^{\wedge} \bar{a}_{n-1}^{1}$ realizes the same type over $A$.

If the conclusion fails, we can find $\bar{c}$ and $\varphi(\bar{x}, \bar{y}) \in \mathbb{L}\left(\tau_{T}\right)$ such that

$(*)_{2} \neg \varphi(\bar{x}, \bar{c}) \in p_{0}$ and $\varphi(\bar{x}, \bar{c}) \in p_{1}$ so $\bar{c} \in{ }^{\ell g(\bar{y})} C$.

Now we choose by induction on $n<\omega$ a sequence $\bar{a}_{n}$ such that

$(*)_{3}$ if $\ell<2$ and $n=\ell \bmod 2$ and we let $A_{n}=\cup \bigcup\left\{\bar{a}_{0}, \ldots, \bar{a}_{n-1}\right\}$ then $\operatorname{tp}\left(\bar{a}_{n}, A_{n} \cup \bar{c}\right)=p_{\ell}^{\left[A, A_{k, n} \cup \bar{c}\right]}$.

Now we can prove by induction on $n<\omega$ that

$(*)_{4}$ the sequences $\bar{a}_{0}^{0 \wedge} \ldots \wedge a_{n-1}^{0}, \bar{a}_{0}^{1 \wedge} \ldots \wedge \bar{a}_{n-1}^{1}$ and $\bar{a}_{0}{ }^{\wedge} \ldots{ }^{\wedge} \bar{a}_{n-1}$ realizes the same type over $A$.

[Why? The first two sequences realizes the same type by $(*)_{1}$. For the induction step, if $n=\ell \bmod 2$, by the definition 5.22 , we have $\bar{a}_{0}^{\ell}{ }^{\wedge}{ }^{\wedge}{ }^{\wedge} \bar{a}_{n-1}^{\ell}{ }^{\wedge} \bar{a}_{n}^{\ell}$ and $\bar{a}_{0}{ }^{\wedge} \ldots{ }^{\wedge} \bar{a}_{n-1} \wedge \bar{a}_{n}$ realizes the same type over $A$.]

So $\left\langle\bar{a}_{n}: n\langle\omega\rangle\right.$ is an indiscernible sequence and $\mathfrak{C} \models \varphi\left[\bar{a}_{n}, \bar{c}\right]$ iff $n$ is odd, contradiction to " $T$ is dependent".

5.26 Conclusion. 1) If $A \subseteq C$ and every $p \in \mathbf{S}^{<\omega}(A)$ is realized in $C$ then $\{p \in$ $\mathbf{S}^{m}(C): p$ does not split over $\left.A\right\}$ has cardinality $\leq\left|\mathbf{S}^{\omega}(A)\right|$ which is $\leq\left(\operatorname{Ded}_{r}(|A|+\right.$ 
$|T|)^{|T|} \leq 2^{|A|+|T|}$ recalling $\operatorname{Ded}_{r}(\mu)=\operatorname{Min}\{\lambda: \lambda$ is regular and every linear order of density $\leq \mu$ has cardinality $\leq \lambda\}$.

2) Also for any finite $\Delta \subseteq \mathbb{L}\left(\tau_{T}\right)$, the set $\left\{p\left\lceil\Delta: p \in \mathbf{S}^{m}(C)\right.\right.$ does not split over $\left.A\right\}$ has cardinality $\leq \operatorname{Ded}_{r}(C)$.

3) If $p \in \mathbf{S}^{m}(C)$ is finitely satisfiable in $A \subseteq C$ then $p$ does not split over $A$.

Proof. Should be clear.

5.27 Definition. For $\ell \in\{1,2\}$, we say $\left\{\bar{a}_{\alpha}: \alpha<\alpha^{*}\right\}$ is $\ell$-independent over $A$ if: we can find $\bar{a}_{\alpha, n}$ (for $\left.\alpha<\alpha^{*}, n<\omega\right\rangle$ such that:

(a) $\bar{a}_{\alpha}=\bar{a}_{\alpha, 0}$

(b) $\left\langle\bar{a}_{\alpha, n}: n<\omega\right\rangle$ is an indiscernible sequence over $A \cup \bigcup\left\{\bar{a}_{\beta, m}: \beta \in \alpha^{*} \backslash\{\alpha\}\right.$ and $m<\omega\}$

(c) $(\alpha) \quad$ if $\ell=1$ then for some $\bar{b}_{n} \in A(n<\omega)$ for every $\alpha<\alpha^{*}$ we have $\left\langle\bar{b}_{n}: n<\omega\right\rangle^{\wedge}\left\langle\bar{a}_{\alpha, n}: n<\omega\right\rangle$ is an indiscernible sequence

( $\beta) \quad$ if $\ell=2$ then for some $\bar{b}_{\alpha, n} \subseteq A$ (for $\alpha<\alpha^{*}, n<\omega$ ), $\left\langle\bar{b}_{\alpha, n}: n<\omega\right\rangle^{\wedge}\left\langle\bar{a}_{\alpha, n}: n<\omega\right\rangle$ is an indiscernible sequence.

We now show that even a very weak version of independence has limitations.

5.28 Claim. 1) For every finite $\Delta \subseteq \mathbb{L}\left(\tau_{T}\right)$ there is $n^{*}<\omega$ such that we cannot find $\bar{\varphi}=\left\langle\varphi_{n}\left(\bar{x}, \bar{a}_{n}\right): n<n^{*}\right\rangle$ such that

$(*)_{\bar{\varphi}}$ for each $n<n^{*}$ there are $m_{n}<\omega$ and $\left\langle\bar{b}_{m, \ell}^{n}: \ell<\omega, m<m_{n}\right\rangle$ and $\left\langle\psi_{m}^{n}\left(\bar{x}, \bar{y}_{n}\right): m<m_{n}\right\rangle$ such that

( $\alpha)\left\langle\bar{b}_{m, \ell}^{n}: \ell<\omega\right\rangle$ is an indiscernible sequence over $\cup\left\{\bar{a}_{k}: k<n^{*}, k \neq n\right\}$

(ß) $\bar{b}_{m, 0}^{n}=\bar{a}_{n}$

$(\gamma)\left\{\psi_{m}^{n}\left(x, \bar{b}_{m, \ell}^{n}\right): \ell<\omega\right\}$ is contradictory for each $n$ and $m<m_{n}$

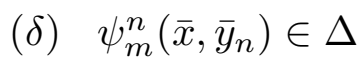

(ع) $\varphi_{n}\left(\bar{x}, \bar{a}_{n}\right) \vdash \bigvee_{m<m_{n}} \psi_{m}^{n}\left(\bar{x}, \bar{a}_{n}\right)$

$(\zeta) \models(\exists \bar{x}) \bigwedge_{n<n^{*}} \varphi_{n}\left(\bar{x}, \bar{a}_{n}\right)$. 
2) We weaken $(\alpha)$ above to $\operatorname{tp}\left(\bar{b}_{m, \ell}^{n}, \bigcup\left\{\bar{a}_{k}: k<n^{*}, k \neq n\right\}\right)=\operatorname{tp}\left(\bar{a}_{n}, \bigcup\left\{\bar{a}_{k}: k<\right.\right.$ $\left.\left.n^{*}, k \neq n\right\}\right)$.

3) Above for some finite $\Delta^{+} \subseteq \mathbb{L}\left(\tau_{T}\right)$, we can in $(\alpha)$ demand only $\Delta^{+}$-indiscernible; also without loss of generality $\varphi_{n}\left(\bar{x}, \bar{y}_{n}\right)=\bigvee_{m<m_{n}} \psi_{m}^{n}\left(\bar{x}, \bar{y}_{n}\right)$.

Proof. 1) [Close to 5.8.] Note

$\circledast$ if $\bar{c} \in \ell^{\ell g(\bar{x})}(\mathfrak{C})$ and $n<n^{*}$ and $\models \varphi_{n}\left(\bar{c}, \bar{a}_{n}\right)$ then for some $\bar{c}^{\prime} \in{ }^{\ell g(\bar{x})}(\mathfrak{C})$ we have

(i) $\operatorname{tp}\left(\bar{c}^{\prime}, \bigcup\left\{\bar{a}_{k}: k<n^{*}, k \neq n\right\}\right)=\operatorname{tp}\left(\bar{c}, \bigcup\left\{\bar{a}_{k}: k<n^{*}, k \neq n\right\}\right)$

(ii) $\operatorname{tp}_{\Delta}\left(\bar{c}, \bar{a}_{n}\right) \neq \operatorname{tp}_{\Delta}\left(\bar{c}^{\prime}, \bar{a}_{n}\right)$.

[Why $\circledast$ holds? Clearly it is enough to find $\bar{b}_{n}^{\prime}$ such that

(i) $\bar{b}_{n}, \bar{b}_{n}^{\prime}$ realize the same type over $\cup\left\{\bar{a}_{k}: k<n^{*}, k \neq n\right\}$

(ii) for some $m<m_{n}$ we have $\psi_{m}^{n}\left(\bar{b}_{n}, \bar{a}_{n}\right) \wedge \neg \psi_{m}^{n}\left(\bar{b}_{n}^{\prime}, \bar{a}_{n}\right)$.

Why does $\bar{b}_{n}^{\prime}$ exist? As $\models \varphi_{n}\left[\bar{c}, \bar{a}_{n}\right]$ by $(\varepsilon)$ for some $m<m_{n}, \models \psi_{n}^{n}\left[\bar{c}, \bar{a}_{n}\right]$ and by $(\alpha)+(\gamma)$, for some $\ell<\omega, b_{n}^{\prime}=\bar{b}_{m, \ell}^{n}$ is as required.]

By repeated use of $\circledast$ we get $m_{\ell}^{*}<m_{\ell}$ such that $\left\langle\psi_{m_{\ell}^{*}}^{n}\left(\bar{x}, \bar{a}_{n}\right): n<n^{*}\right\rangle$ is independent but $\psi_{m_{\ell}^{*}}^{n}\left(\bar{x}, \bar{y}_{n}\right) \in \Delta$ is finite, so $n^{*}$ as required exists.

2),3) Similarly.

5.29 Claim. Assume

(a) $\left\langle\bar{b}_{n}: n<\omega\right\rangle$ is indiscernible over $M$

(b) $\left\{\varphi\left(\bar{x}, \bar{b}_{n}\right): n<\omega\right\}$ is contradictory

(c) $M \prec N, p \in \mathbf{S}(N), \varphi\left(\bar{x}, \bar{b}_{0}\right) \in p$ and $\neg \varphi\left(x, \bar{b}_{n}\right) \in p$ for $n>0$

(d) $N$ is $\|M\|^{+}$-saturated.

Then for some $\left\langle\bar{b}_{n}^{\prime}: n<\omega\right\rangle$ we have

( $\alpha)\left\langle\bar{b}_{n}^{\prime}: n<\omega\right\rangle$ is indiscernible over $M$ based on $M, \bar{b}_{n}^{\prime} \subseteq N$

( $\beta) \bar{b}_{0}^{\prime} \in\left\{\bar{b}_{0}, \bar{b}_{1}\right\}$

$(\gamma) \varphi\left(\bar{x}, \bar{b}_{0}^{\prime}\right) \equiv \neg \varphi\left(\bar{x}, \bar{b}_{1}^{\prime}\right)$ belongs to $p$.

Proof. Easy. 
5.30 Definition. 1) For $p \in \mathbf{S}^{m}(M)$ let $\mathscr{E}(p)$ be the set of pairs $(\varphi(\bar{x}, \bar{y})$, e) such that

(a) e is a definable equivalence relation on ${ }^{\ell g(\bar{y})} M$ in $M$

(b) if $\bar{b}_{1} \mathbf{e} \bar{b}_{2}$ then $\varphi\left(\bar{x}, \bar{b}_{1}\right) \in p \Leftrightarrow \varphi\left(\bar{x}, \bar{b}_{2}\right) \in p$.

2) $\mathscr{E}_{\text {tp }}^{\prime}(p)$ is defined similarly by e is definable by types.

5.31 Claim. Assume $\varphi=\varphi(x, \bar{y}), M \prec N, N$ is $\|M\|^{+}$-saturated and $p \in \mathbf{S}(N)$. Then we cannot find $\left\{D_{i}: i<n_{\varphi}\right\}$, a set of ultrafilters over ${ }^{\ell}(\bar{y})(N)$ pairwise orthogonal (see below) with $p_{i}=\operatorname{Av}\left(N, D_{i}\right)$ such that $p(x) \cup p_{i}\left(\bar{y}_{0}\right) \cup p_{i}\left(\bar{y}_{1}\right) \cup$ $\left\{\varphi\left(\bar{x}, \bar{y}_{1}\right), \neg \varphi\left(x, \bar{y}_{0}\right)\right\}$ is consistent for $i<n_{\varphi}$.

Now we deal with Orthogonality.

5.32 Definition. 1) Two complete types $p(\bar{x}), q(\bar{y})$ over $A$ are weakly orthogonal if $p(\bar{x}) \cup q(\bar{y})$ is a complete type over $A$.

2) Assume $\overline{\mathbf{b}}_{1}, \overline{\mathbf{b}}_{2}$ are endless indiscernible sequences. We say $\overline{\mathbf{b}}_{1}, \overline{\mathbf{b}}_{2}$ are orthogonal and write $\overline{\mathbf{b}}_{1} \perp \overline{\mathbf{b}}_{2}$ if:

for every set $A$ which includes $\overline{\mathbf{b}}_{1} \cup \overline{\mathbf{b}}_{2}, \operatorname{Av}\left(A, \overline{\mathbf{b}}_{1}\right), \operatorname{Av}\left(A, \overline{\mathbf{b}}_{2}\right)$ are weakly orthogonal

3) $\overline{\mathbf{b}}_{1}$ is strongly orthogonal to $\overline{\mathbf{b}}_{2}, \mathbf{b}_{1} \frac{\perp}{\mathrm{st}} \mathbf{b}_{2}$ if it is orthogonal to every endless indiscernible sequence $\overline{\mathbf{b}}_{2}^{\prime}$ of finite distance from $\overline{\mathbf{b}}_{2}$ (see $[$ Sh $715,1.11=\mathrm{np} 1.4 \mathrm{~B}](2)$. 4) An endless indiscernible sequence $\overline{\mathbf{b}}_{1}$ is orthogonal to $\varphi(x, \bar{a})$ if it is orthogonal to every endless indiscernible sequence $\overline{\mathbf{b}}_{2}=\left\langle b_{2, \alpha}: \alpha<\delta\right\rangle$ such that $b_{2, \alpha} \in \varphi(\mathfrak{C}, \bar{a})$ for every $\alpha<\delta$. Similarly for $\varphi_{1}\left(x, \bar{a}_{1}\right), \varphi_{2}\left(x, \bar{a}_{2}\right)$. If $\operatorname{tp}\left(b_{\alpha}, A\right), \operatorname{tp}\left(\cup\left\{\bar{b}_{\beta}: \beta \in \alpha^{*} \backslash\{\alpha\},\right)\right.$ for $\alpha<\alpha^{*}$ then every $p \in \mathbf{S}^{m}(A)$ is weakly orthogonal to $\operatorname{tp}\left(\bar{b}_{\alpha}, A\right)$ for all but $\leq|T|$ of the ordinal $\alpha<\alpha^{+}$.

$5) \overline{\mathbf{b}}$ is based on $A$ if $\overline{\mathbf{b}}$ is an indiscernible sequence and $C_{A}(\overline{\mathbf{b}})$ (see [Sh 715] or [Sh:93]) has boundedly many conjugations over $A$.

6) If $\overline{\mathbf{b}}_{1} \frac{\perp}{\text { st }} \overline{\mathbf{b}}_{2}$ and $\overline{\mathbf{b}}_{\ell}^{\prime}$ is a neighbor (see $[\mathrm{Sh} 715,1.11=\mathrm{np} 1.4 \mathrm{~B}]$ ) to $\overline{\mathbf{b}}_{\ell} \underline{\text { then }} \overline{\mathbf{b}}_{1}^{\prime}$ is strongly orthogonal to $\overline{\mathbf{b}}_{2}^{\prime}$.

5.33 Claim. 1) Orthogonality is symmetric relation.

2) If $\mathbf{b}_{1}, \mathbf{b}_{2}$ are orthogonal then they are perpendicular (see Definition 2.2).

5.34 Example: In $\operatorname{Th}(\mathbb{R},<)$, different initial segments are orthogonal, even two disjoint intervals. For $(\mathbb{R}, 0,1,+, \times)$ the situation is different: any two non trivial intervals are "the same". 
5.35 Claim. 1) Assume $\lambda=\lambda^{<\lambda}, I$ is a dense linear order with neither first nor last element and $\overline{\mathbf{b}}=\left\langle\bar{b}_{t}: t \in I\right\rangle$ an indiscernible sequence. If $|I|=\lambda$, then there is $M \supseteq \overline{\mathbf{b}}$ which is $\lambda$-saturated and $\lambda$-atomic over $\overline{\mathbf{b}}$.

2) If $p \in \mathbf{S}^{m}(\overline{\mathbf{b}})$ is $\lambda$-isolated then it is $|T|^{+}$-isolated.

5.36 Question: $M$ is $\mu$-minimal over $\overline{\mathbf{b}}$, i.e. over $B:=\cup\left\{\bar{b}_{t}: t \in I\right\}$ (i.e. there is no $N, \overline{\mathbf{b}} \subseteq N \prec M, N \neq M$ such that $N$ is $\mu^{+}$-saturated) iff there is no indiscernible sequence over $\overline{\mathbf{b}}$ which is $|T|^{+}$-isolated over $\overline{\mathbf{b}}$.

5.37 Question: If $\operatorname{Av}\left(M, \overline{\mathbf{b}}_{1}\right), \operatorname{Av}\left(M, \overline{\mathbf{b}}_{2}\right)$ (or with $D$ 's) are weakly orthogonal and are perpendicular, then are they orthogonal?

5.38 Question: On the set of elements or sequences realizing the type $\operatorname{Av}\left(\overline{\mathbf{b}}_{1}, \overline{\mathbf{b}}\right), \overline{\mathbf{b}}$ an endless indiscernible sequence, can we define a dependence relation similar enough to the stable case (so if $\overline{\mathbf{b}}^{\wedge} \overline{\mathbf{b}}_{2}$ is indiscernible then $\overline{\mathbf{b}}_{1}$ is independent).

5.39 Question: For each of the following conditions can we characterize the dependent theories which satisfy it?

(a) for any two non-trivial indiscernible sequences $\overline{\mathbf{b}}_{1}, \overline{\mathbf{b}}_{2}$, we can find $\overline{\mathbf{b}}_{\ell}^{\prime}$ of finite distance from $\overline{\mathbf{b}}_{\ell}$ (see [Sh 715], for $\ell=1,2$ ) such that $\overline{\mathbf{b}}_{1}^{\prime}, \overline{\mathbf{b}}_{2}^{\prime}$ are not orthogonal

(b) any two non-trivial indiscernible sequences of singletons have finite distance?

(c) $T$ is $\operatorname{Th}(\mathbb{F}), \mathbb{F}$ a field (so this class includes the $p$-adics various reasonable fields with valuations and closed under finite extensions). 


\section{REFERENCES.}

[BaPo98] Yerzhan Baisalov and Bruno Poizat. Paires de structures o-minimales. Journal of Symbolic Logic, 63:570-578, 1998.

[BBSh 815] Bektur Baizhanov, John Baldwin, and Saharon Shelah. Subsets of superstable structures are weakly benign. Journal of Symbolic Logic, 70:142-150, 2005. math.LO/0303324.

[CoSh:919] Moran Cohen and Saharon Shelah. Stable theories and representation over sets. Mathematical Logic Quarterly, submitted. 0906.3050.

[Cmf77] W. Wistar Comfort. Ultrafilters: some old and some new results. Bulletin of the American Mathematical Society, 83:417-455, 1977.

[FiSh:E50] Eyal Firstenberg and Saharon Shelah. Perpendicular Indiscernible Sequences in Real Closed Fields.

[Ke87] H. Jerome Keisler. Measures and forking. Annals of Pure and Applied Logic, 34:119-169, 1987.

[Po81] Bruno Poizat. Théories instables. Journal of Symbolic Logic, 46:513$522,1981$.

[Sh 886] Saharon Shelah. Definable groups for dependent and 2-dependent theories. Journal of Symbolic Logic, submitted. math.LO/0703045.

[Sh 877] Saharon Shelah. Dependent $T$ and Existence of limit models. Tbilisi Mathematical Journal, submitted. math.LO/0609636.

[Sh:900] Saharon Shelah. Dependent theories and the generic pair conjecture. Communications in Contemporary Mathematics, submitted. math.LO/0702292.

[Sh:F705] Saharon Shelah. Representation over orders of elementary classes.

[Sh 839] Saharon Shelah. Stable frames and weights. Preprint.

[Sh 863] Saharon Shelah. Strongly dependent theories. Israel Journal of Mathematics, accepted. math.LO/0504197.

[Sh:F660] Saharon Shelah. Toward the main gap by fatness, a modest beginning.

[Sh 3] Saharon Shelah. Finite diagrams stable in power. Annals of Mathematical Logic, 2:69-118, 1970.

[Sh:93] Saharon Shelah. Simple unstable theories. Annals of Mathematical Logic, 19:177-203, 1980. 
[Sh:c] Saharon Shelah. Classification theory and the number of nonisomorphic models, volume 92 of Studies in Logic and the Foundations of Mathematics. North-Holland Publishing Co., Amsterdam, xxxiv+705 pp, 1990.

[Sh 715] Saharon Shelah. Classification theory for elementary classes with the dependence property - a modest beginning. Scientiae Mathematicae Japonicae, 59, No. 2; (special issue: e9, 503-544):265-316, 2004. math.LO/0009056.

[Sh 876] Saharon Shelah. Minimal bounded index subgroup for dependent theories. Proceedings of the American Mathematical Society, 136:10871091, 2008. math.LO/0603652.

[Sh:906] Saharon Shelah. No limit model in inaccessibles. CRM Proceedings and Lecture Notes, 53:277-290, 2011. 0705.4131. 